\title{
THE FEASIBILITY OF SODAR WIND PROFILE MEASUREMENTS FROM AN OCEANOGRAPHIC BUOY
}

\author{
By \\ Allison M. Berg \\ B.S., United States Naval Academy, 2004 \\ Submitted in partial fulfillment of the requirements of the degree of \\ Master of Science \\ at the \\ MASSACHUSETTS INSTITUTE OF TECHNOLOGY \\ and the \\ WOODS HOLE OCEANOGRAPHIC INSTITUTION
}

September 2006

(C) 2006 Allison M. Berg

All rights reserved.

The author hereby grants to MIT and WHOI permission to reproduce paper and electronic copies of this thesis in whole or in part and to distribute them publicly.

Author

Joint Program in Oceanography/Applied Ocean Science and Engineering Massachusetts Institute of Technology and Woods Hole Oceanographic Institution

September 2006

Certified by

Eugene A. Terray

Research Specialist, Woods Hole Oceanographic Institution Thesis Supervisor

Accepted by

Henrik Schmidt

Professor of Mechanical and Ocean Engineering Chairman, Joint Committee for Applied Ocean Science and Engineering Massachusetts Institute of Technology and Woods Hole Oceanographic Institution

Accepted by

Lallit Anand

Professor of Mechanical Engineering Chairman, Department of Mechanical Engineering Graduate Committee Massachusetts Institute of Technology 


\title{
THE FEASIBILITY OF SODAR WIND PROFILE MEASUREMENTS FROM AN OCEANOGRAPHIC BUOY
}

\author{
by \\ Allison M. Berg \\ Submitted in partial fulfillment \\ of the requirements for the degree of \\ Master of Science \\ at the \\ MASSACHUSETTS INSTITUTE OF TECHNOLOGY \\ and the \\ WOODS HOLE OCEANOGRAPHIC INSTITUTION \\ September 19, 2006
}

\begin{abstract}
This thesis explores the feasibility of making wind speed profile measurements from an oceanographic buoy using a Doppler sodar.

In the fall of 2005, we deployed a Scintec SFAS sodar on an ASIS buoy. Roughly one week of buoy motion data and one day of sodar observations were collected. Data from both this deployment, and the Martha's Vineyard Coastal Observatory, were used in conjunction with models to predict sodar performance. Results are compared for an ASIS and a 3-meter discus buoy. We also predict the yearly average probability of sodar data availability in the presence of buoy motion. We show that buoy tilting in response to wave forcing is the main factor affecting sodar performance. Our results strongly suggest that ASIS is a suitable platform for sodar measurements at sea.
\end{abstract}

Thesis Supervisor: Dr. Eugene A. Terray

Title: Research Specialist 


\section{Aknowledgements}

I wish to express my most sincere gratitude to Gene Terray for his knowledge, patience, and guidance through this project. His dedication to helping me during my time here has made this thesis a success.

I would also like to thank the US Navy for the opportunity to study at MIT and WHOI, as well as the Massachusetts Technology Collaborative for funding the fieldwork conducted as part of this thesis.

Neil McPhee, Ben Allen, Will Ostrom, Steve Faluotico, Ed Hobart, Jim Doutt, Megan Carrol, and the WHOI rigging, carpentry, and mechanical shops - particularly Geoff, Paul and Carlos - for all of their help throughout this project.

Finally, I extend a thank you to my family for their unconditional support and love throughout all my endeavors. 


\section{Table of Contents}

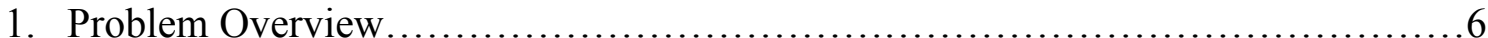

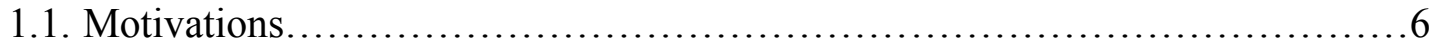

1.2. Doppler profiling instruments..........................................

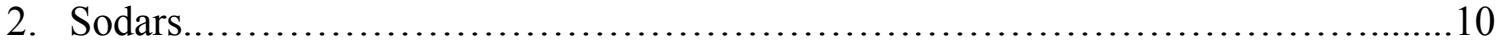

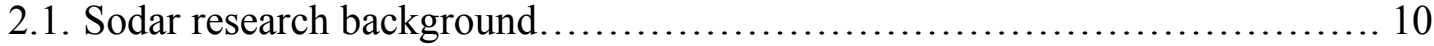

2.2. Sodar theory of operation............................................... 11

2.3. Spectral first moment................................................ 12

2.4. Variance in the Doppler frequency estimate............................. 13

2.5. Scintec SFAS ...................................................... 14

2.6. Scintec beampattern calculation...................................... 15

3. Sodar platforms..................................................... 19

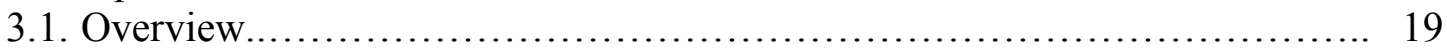

3.2. Three meter discus buoy characteristics............................... 20

3.3. ASIS design characteristics......................................... 22

3.4. ASIS instrumentation and data acquisition............................. 25

4. Analysis of ASIS platform dynamics..................................... 27

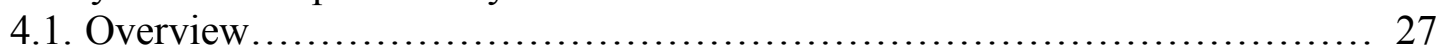

4.2. Wind and wave conditions......................................... 27

4.3. Measured ASIS response to wave forcing............................... 31

4.4. Comparison of the modeled and measured ASIS tilt response............... 35

4.5. Modeled ASIS spectral response to waves................................ 37

4.6. Modeled ASIS temporal response to waves........................... 41

4.7. Wave overtopping probabilities........................................ 42

4.8. ASIS tilt response to sub-waveband frequencies......................... 43

4.9. Wave band tilts....................................................... 45

4.10 ASIS drift response to combined wind and wave forcing................... 47

5. Sodar performance on ASIS and conclusions................................. 55

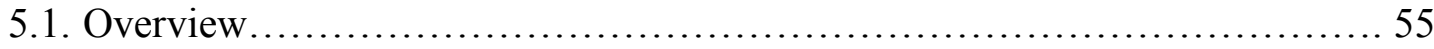

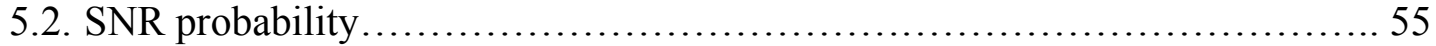

5.3. Overlap percentages..................................................... 56

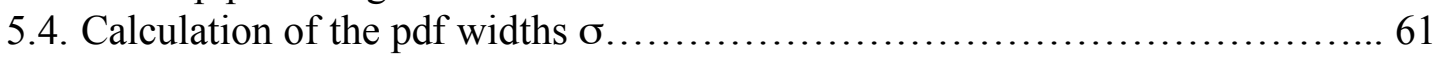

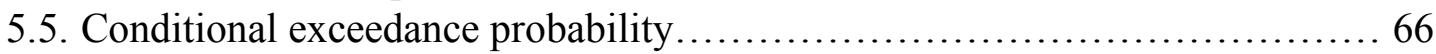

5.6. Yearly average data availability due to motion.............................69

5.7. Conclusions..................................................... 73

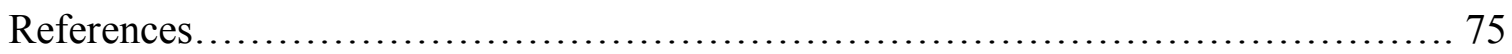

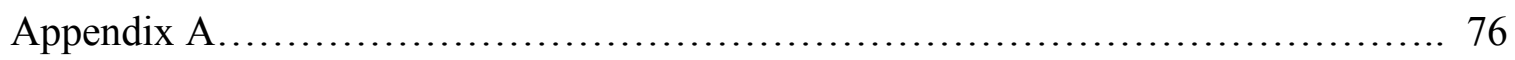

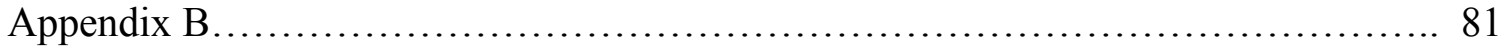

Appendix C................................................................. 89

Appendix D................................................................. 90

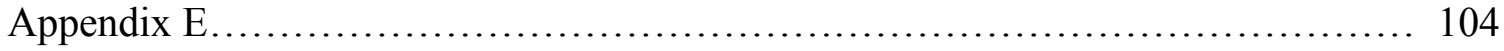

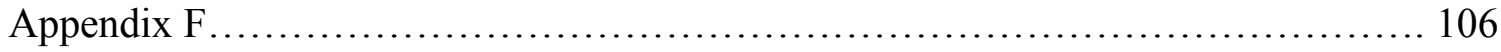

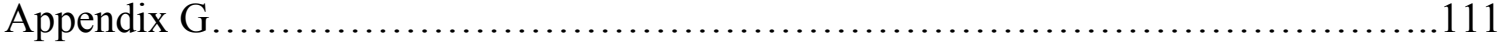




\section{Chapter 1}

\section{Problem Overview}

\subsection{Motivations}

In the present day, fixed meteorological towers are the most commonly used platform from which to make wind measurements in the marine atmospheric boundary layer (MABL). The determining factors in the cost of a meteorological tower are the depth of the water, the air-side height of the tower and the typical wave climate of the chosen location. As a result, it is not economical to build a tower that extends far above the mean water level or one that is situated in deep water. For example, it can cost between $\$ 1.5-$ $2 \mathrm{M}$ to construct a $60 \mathrm{~m}$ tower in $10 \mathrm{~m}$ of water. From the point of view of site characterization for wind power generation, fixed meteorological towers clearly are limited to measuring winds at a fixed location, so there is no possibility of exploring different or better sites. Also, towers generally are not high enough to measure winds over the span of large wind turbine rotors. For example, the General Electric 3.6sl offshore wind turbine has a rotor diameter of $111 \mathrm{~m}$. Fixed meteorological towers also are inadequate when it comes to capturing the full scope of MABL physics. In the summer months along the Atlantic continental shelf, stable stratification in the atmosphere can lead to the formation of low-level jets (Vickers and Mahrt, 2004). An example is shown in Figure 1.1, which is taken from the CBLAST - Low experiment conducted off the south coast of Martha's Vineyard, Massachusetts in August 2001. The wind speed profile shows a strong shear over the lower $100 \mathrm{~m}$, reaching a maximum of about $14 \mathrm{~m} / \mathrm{s}$ at $110 \mathrm{~m}$, where it reverses direction. Because such jets can produce loads that will shorten the life of wind turbine blades, it is important to be able to characterize them and determine their frequency of occurrence, and hence wind profiles over heights of order $150 \mathrm{~m}$ are necessary.

In this thesis we will study the feasibility of using Doppler sodar, deployed on an oceanographic buoy, to profile winds over several hundred meters with a range resolution of several meters. 

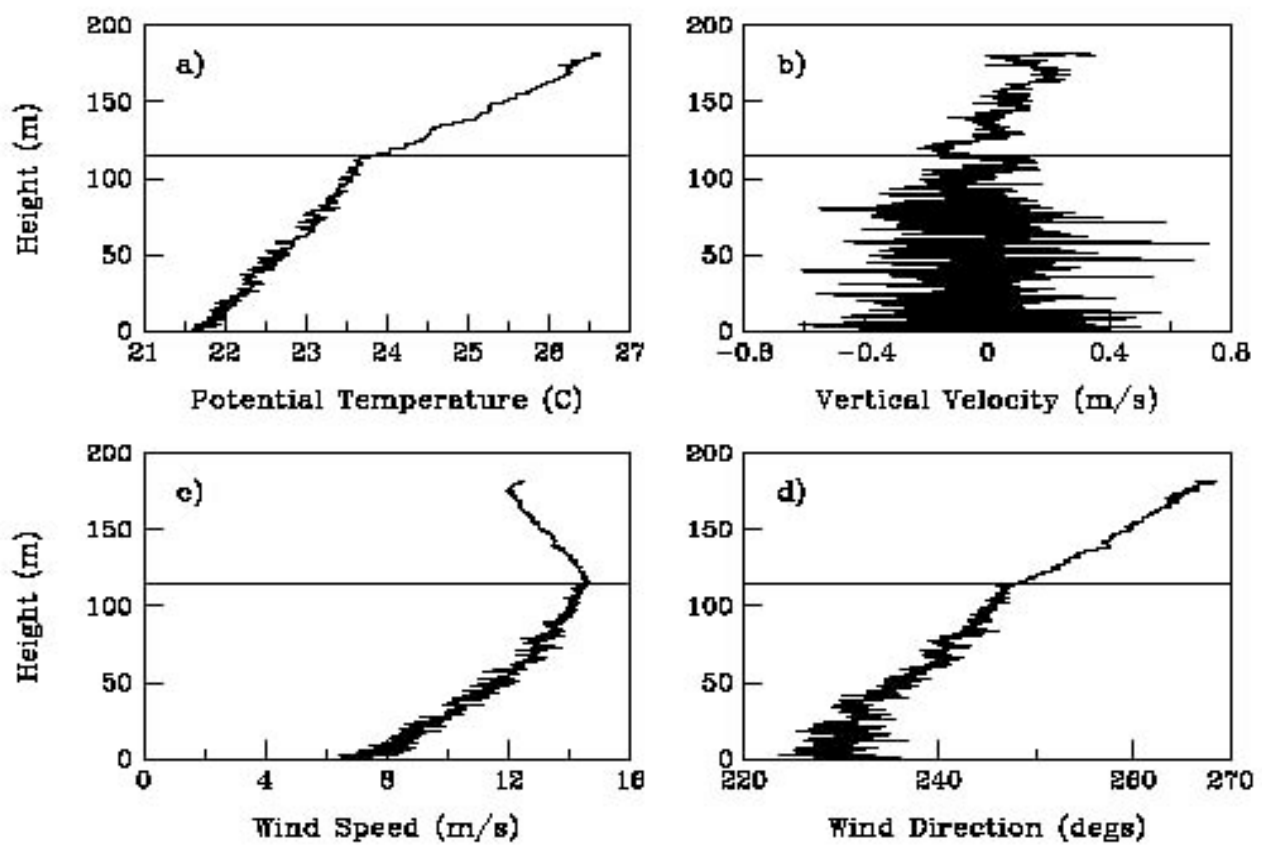

Figure 1.1 Data taken from the CBLAST- Low experiment conducted off the south coast of Martha's Vineyard in August 2001 (Vickers and Mahrt, 2004).

\subsection{Doppler profiling instruments}

In this section we survey approaches to measuring wind profiles over several hundred meters. Three different kinds of instruments are capable in principle of sensing wind profiles: Doppler lidars, radars, and sodars.

Doppler lidar (laser radar) measures the Doppler shift of radiation that is scattered by natural particles such as dust and water droplets.

Two primary varieties of lidars exist: pulsed and continuous wave (CW). Pulsed lidars have an along - beam range resolution that is constant with respect to range, but typically cost $\$ 1 \mathrm{M}$ or more because the pulsed lasers and digitizer are expensive. In contrast, $\mathrm{CW}$ lidars tend to be much less expensive. For example, QinetiQ makes a CW infrared lidar called the ZephIR (see Figure 1.2), that is based on readily available parts from the telecom industry, and sells for $\sim \$ 180 \mathrm{~K}$. It uses a conically scanned laser at a wavelength of $1.55 \mu \mathrm{m}$. The ZephIR is a compact package that weighs $40 \mathrm{~kg}$ and consumes about 
$100 \mathrm{~W}$. The system can make wind speed measurements up to $200 \mathrm{~m}$, and data from it has compared very well with cup anemometer measurements (Jorgenson et. al., 2004). The main difficulty in the CW approach is how to determine range. The ZephIR uses a mechanically scanned focal point. However, in this case the range cell increases as the square of the range. So, for example, whereas at $50 \mathrm{~m}$ the range resolution is $4 \mathrm{~m}$, at $200 \mathrm{~m}$ the range resolution has decreased to $60 \mathrm{~m}$ (Jorgenson et. al., 2004).

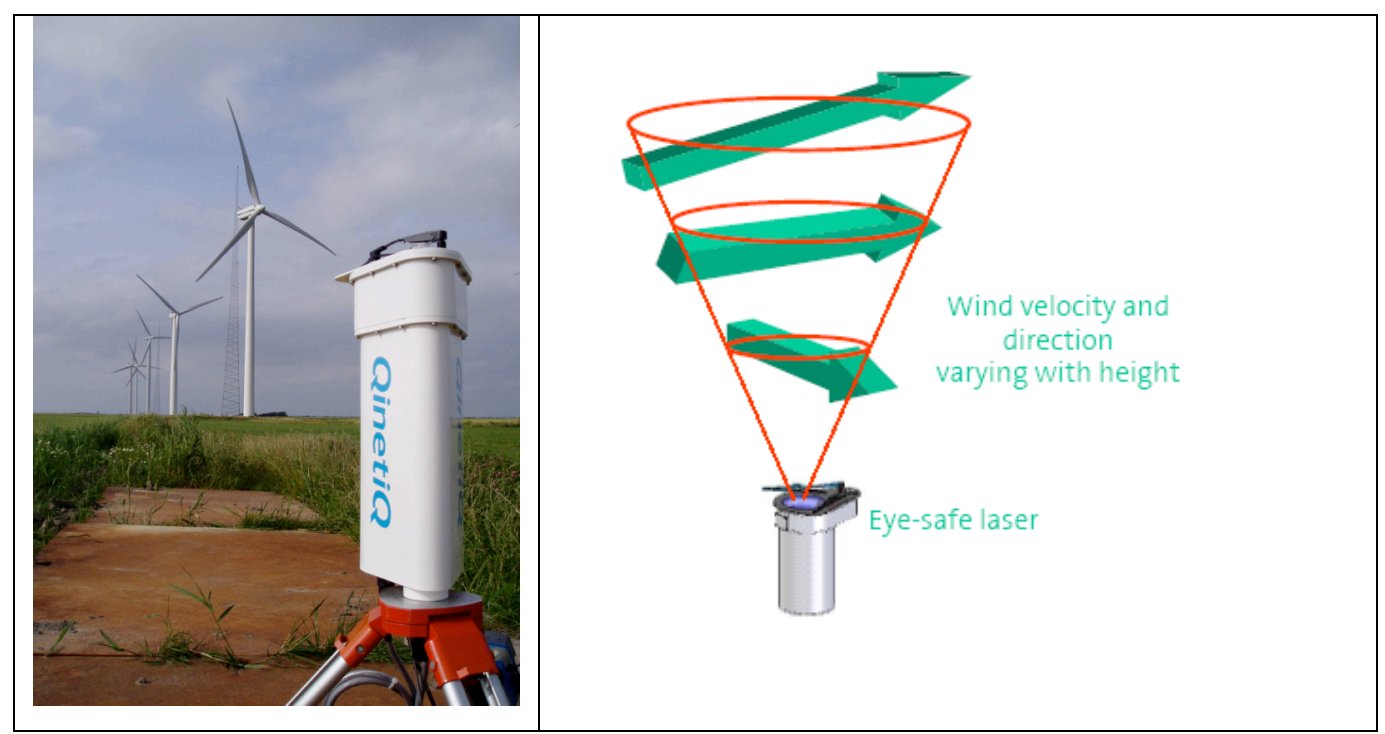

Figure 1.2 ZephIR lidar and its principle of operation (figure courtesy of D.A. Smith, QinetiQ Ltd.)

A second way to obtain wind profiles in the MABL is via Doppler radar. These devices emit electromagnetic energy into the atmosphere and detect the Doppler shift of the returned signal. NOAA currently operates a network of Doppler radars in the United States that profile winds up to several kilometers. Although these instruments are commercially available, they have a range resolution of $60-100 \mathrm{~m}$, making them unsuitable for our application. At the present time, there are no commercially-available radars having a range resolution of order a few meters over the lowest $200 \mathrm{~m}$.

ProSensing has developed a prototype radar called the MBL-profiler (see Figure 1.3) that can be used from a small boat or buoy. The MBL-profiler uses a four-beam microstrip antenna and can probably profile over several hundred meters. Although not commercially available, when in regular production, such a radar would likely cost $\mathrm{O}(\$ 200 \mathrm{~K})$. 


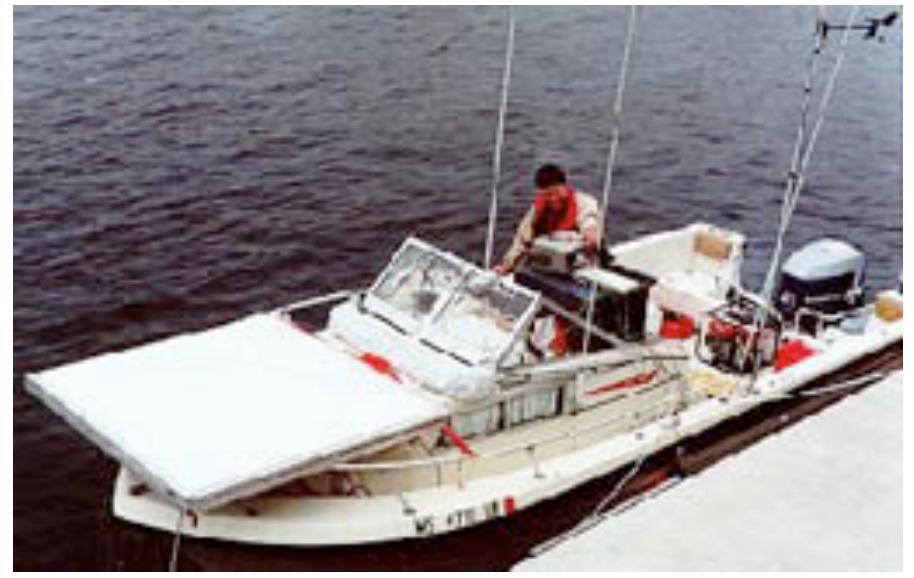

Figure 1.3 ProSensing's MBL-profiler rigged aboard a small boat

Doppler sodars work by scattering an acoustic pulse from sound speed fluctuations in the atmosphere and estimating wind speed at each range. Wind profiles are computed from the Doppler shift which is computed from the frequency of the returned signal (more specifics are discussed later). Several compact, low-power, sodars are available commercially. For example, Scintec and Remtech both build flat phased array sodars that require $\sim 100 \mathrm{~W}$ (transmit power). These instruments can profile over $\sim 150 \mathrm{~m}$ with $5 \mathrm{~m}$ range resolution and are comparatively priced at roughly $\$ 60 \mathrm{~K}$. Since we have access to the Scintec SFAS model we will focus on that sodar here. 


\section{Chapter 2}

\section{Sodars}

\subsection{Sodar research background}

Sodars have a long history of use on land and have been successfully used in many pollution and boundary layer studies. Numerous comparison experiments have been performed to validate the accuracy of Doppler sodar use over land (Crescenti, 1997). However, few marine-based sodar studies have been conducted and the results have been mixed. It has been suggested that one reason for the poor results reported is that the marine environment may not provide enough scattering strength (Fairall et.al., 1997). However, in a 1 day study, Barthelmie et. al. (2003) mounted a sodar on a moored ship near Vindeby, Denmark and got good results. Their Aerovironment 4000 mini-sodar obtained results that were not significantly different than those collected during landbased experiments with the same instrument (Coelingh et. al. 2000).

A. L. Rogers (2006) has also obtained good quality results using a sodar in the marine environment. In the fall of 2005, his group operated their ART-VT1 sodar from the WHOI Air Sea Interaction Tower (ASIT) south of Martha's Vineyard. The sodar was deployed from 22 September 2005 to 18 November 2005 and got good data return. Figure 2.1 shows a plot of percentage of acceptable 10-minute averages as a function of range. For heights up to $80 \mathrm{~m}$ above the sodar, at least $50 \%$ of the 10 minute averaged spectra yielded acceptable Doppler estimates.

The experiences of Barthelmie et. al. (2003) and A. L. Rogers (2006) suggest that there is potential for obtaining quality data from a sodar in the marine environment. However, the sodar studies performed by the previously mentioned groups were both conducted from platforms where motion was negligible. Rogers (2006) recorded sodar data from a tower, whereas Barthelmie et. al. (2003) made measurements from a ship anchored with a threepoint mooring in low sea states. No one has attempted to collect sodar data from a moving platform in the marine environment. This thesis explores the feasibility of obtaining quality wind profile data from an oceanographic buoy. 


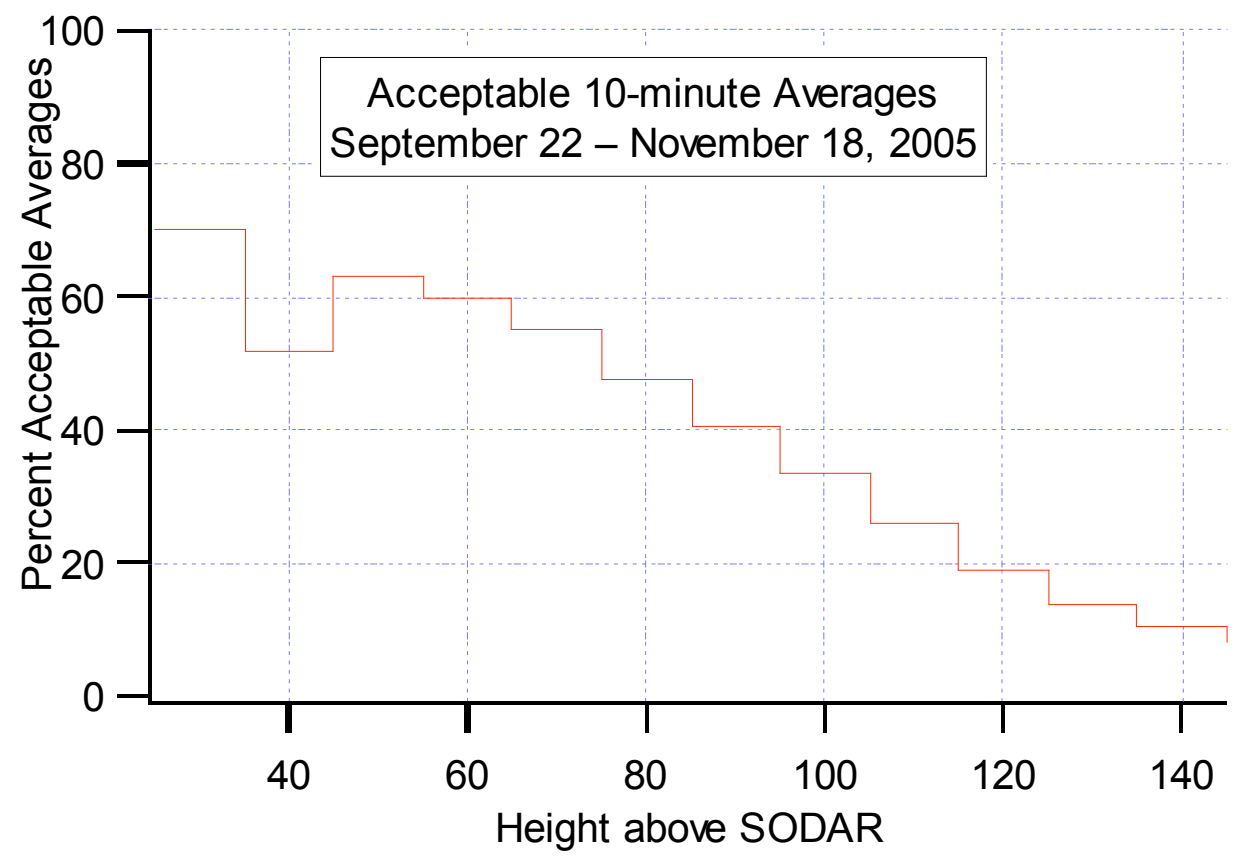

Figure 2.1 Percentage of acceptable 10 minute averages (A. L Rogers, 2006).

\subsection{Sodar theory of operation}

A sodar works by emitting an acoustic pulse that is backscattered from sound speed inhomogeneities in the atmosphere. Monostatic sodars have their transmitter and receiver in the same location so that only direct backscatter can be measured. Backscatter is detected if turbulent eddies fulfill the Bragg condition (i.e. the spatial wavenumber of the turbulent temperature fluctuations twice the acoustic wavenumber). Sound can also be backscattered from density discontinuities. The Doppler shift is computed from the return frequency of the backscattered signal, The magnitude of the frequency shift is

$$
\delta f=-2 \frac{u_{r}}{c} f_{e}
$$

where $f_{e}$ is the frequency of the emitted pulse, $u_{r}$ is the component of the wind velocity along the beam, and $c$ is the speed of sound (Pierce, 1981). For a beam tilted at an angle $\theta$ with respect to the vertical, the Doppler shift is 


$$
\delta f=-2 \frac{u}{c} f_{e} \sin (\theta)-2 \frac{w}{c} f_{e} \cos (\theta)
$$

where $u$ and $w$ are the horizontal and vertical components of the wind in the plane defined by the vertical and the beam. Using (2.2), easterly $(u)$, northerly $(v)$, and vertical wind components $(w)$ can be calculated from frequency shifts measured by north - $(\mathrm{N})$, east - (E) and vertical-pointing beams (V) as

$$
\begin{aligned}
& u=-\frac{\delta f_{E}}{2 f_{E}} \frac{c}{\sin \left(\theta_{E}\right)}+\frac{\delta f_{V}}{2 f_{V}} \frac{c}{\tan \left(\theta_{E}\right)} \\
& v=-\frac{\delta f_{N}}{2 f_{N}} \frac{c}{\sin \left(\theta_{N}\right)}+\frac{\delta f_{V}}{2 f_{V}} \frac{c}{\tan \left(\theta_{N}\right)} \\
& w=-\frac{\delta f_{V}}{2 f_{V}} c
\end{aligned}
$$

\subsection{Spectral first moment}

This section describes how to estimate Doppler shift numerically via a spectral first

moment calculation. With an estimate of the spectral mean frequency $(\bar{f})$, we compute Doppler shift using the following

$$
\delta f=f_{e}-\bar{f}
$$

The first moment of the measured spectrum, $S_{M}$, yields the mean frequency, $\bar{f}_{B}$

$$
\bar{f}_{B}=\frac{\int f S_{M}(f) d f}{\int S_{M}(f) d f}=\frac{\int\left(f-\frac{1}{2} f_{N}\right) S_{M} d f}{\int S_{M} d f}+\frac{1}{2} f_{N}
$$


where $S_{M}$ is the measured spectrum of the returned pulse and $f_{N}$ is the Nyquist frequency. This estimate is biased because the spectrum of the noise is included in the calculation.

Equation (2.7) can be written as

$$
\bar{f}_{B}=\alpha \bar{f}+\frac{1}{2}(1-\alpha) f_{N}
$$

where

$$
\alpha=\frac{\int S d f}{\int S_{M} d f}=1-\frac{\int N}{\int S_{M}}=1-\frac{1}{S N R_{M}}=\frac{S N R_{M}-1}{S N R_{M}}
$$

From equation (2.8), we estimate the mean frequency as

$$
\bar{f}=\left[\bar{f}_{B}-\frac{1}{2}(1-\alpha) f_{N}\right] / \alpha .
$$

\subsection{Variance in the Doppler frequency estimate}

In this section, we use the ratio of signal over noise to assess the quality of our sodar data. For a given SNR we can calculate how much variance we have in the Doppler frequency estimate. The Cramer-Rao bound for an unbiased estimator is:

$$
\sigma_{f}=(2 \pi T)^{-1}\left(1+36 / S N R+30 / S N R^{2}\right)^{1 / 2}
$$

(Theriault,1986) where $\sigma_{f}$ is Doppler frequency variance, $T$ is the pulse length and $S N R$ is the signal-to-noise ratio. The variance of Doppler frequency, non-dimensionalized by the pulse length, $T$, and multiplied by $2 \pi$, is shown in Figure 2.2. As SNR increases, $\sigma_{f} 2 \pi T$ decreases rapidly and asymptotes to unity. 


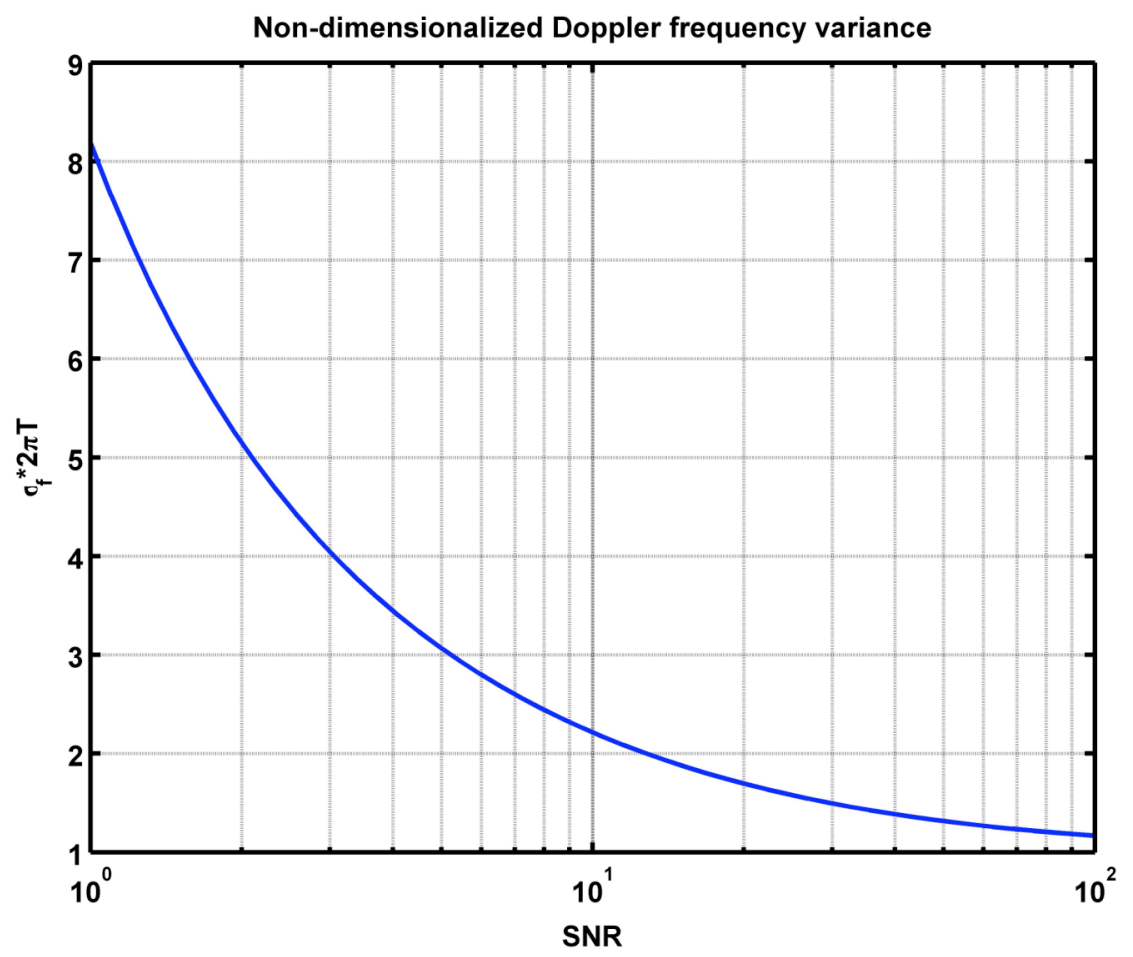

Figure 2.2 Non-dimensionalized Doppler frequency variance versus SNR. The range resolution is $\Delta R=\frac{1}{2} C T$.

For $C=330 \mathrm{~m} / \mathrm{s}$, a $30 \mathrm{~ms}$ pulse has a range resolution of $5 \mathrm{~m}$. Hence for SNR between one and infinity, $\sigma_{f} \sim 5 \sim 40 \mathrm{~Hz}$. The corresponding uncertainty for $f_{e} \sim 330 \mathrm{~Hz}$ in the radial velocity is $0.25-2 \mathrm{~m} / \mathrm{s}$.

\subsection{Scintec SFAS}

The Scintec SFAS is a compact sodar capable of profiling winds up to $200 \mathrm{~m}$ with $\mathrm{O}(5 \mathrm{~m})$ range resolution. The sodar, shown in Figure 2.3, is a 64 element array that can synthesize up to nine shaded beams in transmit and receive mode at angles of $0^{\circ}, \pm 19^{\circ}$ and $\pm 24^{\circ}$. The SFAS can emit up to 10 tones (acoustic pulses) in the frequency range of 2525 to $4850 \mathrm{~Hz}$. The instrument is relatively portable in that it weighs only $11.5 \mathrm{~kg}$, and measures $44 \times 42 \times 16 \mathrm{~cm}$. It sits in a baffle (Figure B.2) that opens at an angle of $25.9^{\circ}$. The baffle serves to mitigate the effect of high angle noise on the received signal. 


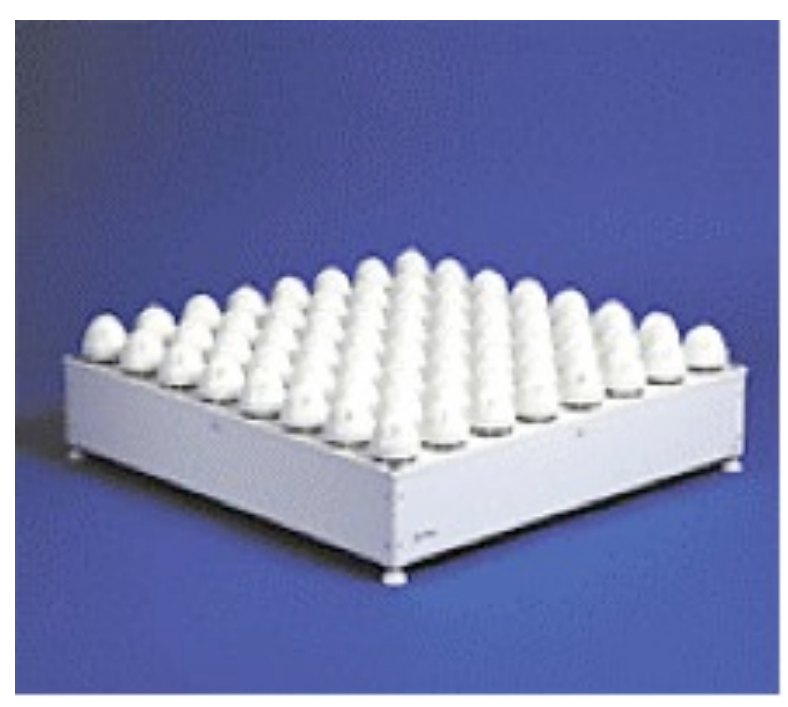

Figure 2.3 Scintec SFAS sodar.

\subsection{Scintec beampattern calculation}

In this section we compute the Scintec SFAS beampattern. A description of the relevant theory is given in Appendix A. The directivity of the vertical $\left(0^{\circ}\right)$ and steered beams $( \pm$ $19^{\circ}, \pm 24^{\circ}$ ) is slightly different. We compare a 1-dimensional slice through the beampatterns for the vertical and steered $19^{\circ}$ beam case at the instrument's middle tone, $3568 \mathrm{~Hz}$ (see Figure 2.4). In the figure, the steered beampattern for the $19^{\circ}$ case is centered at $0^{\circ}$ so that it can easily be compared to the vertical beam. Only positive angles of the beampattern are shown as the beampattern is a mirror image at the corresponding negative angles. Since the SFAS beamforms both the transmit and receive beams, we show the product of the transmit and receive beam intensity patterns (i.e. the 2-way intensity) normalized to have a maximum value of unity along the central axis. 


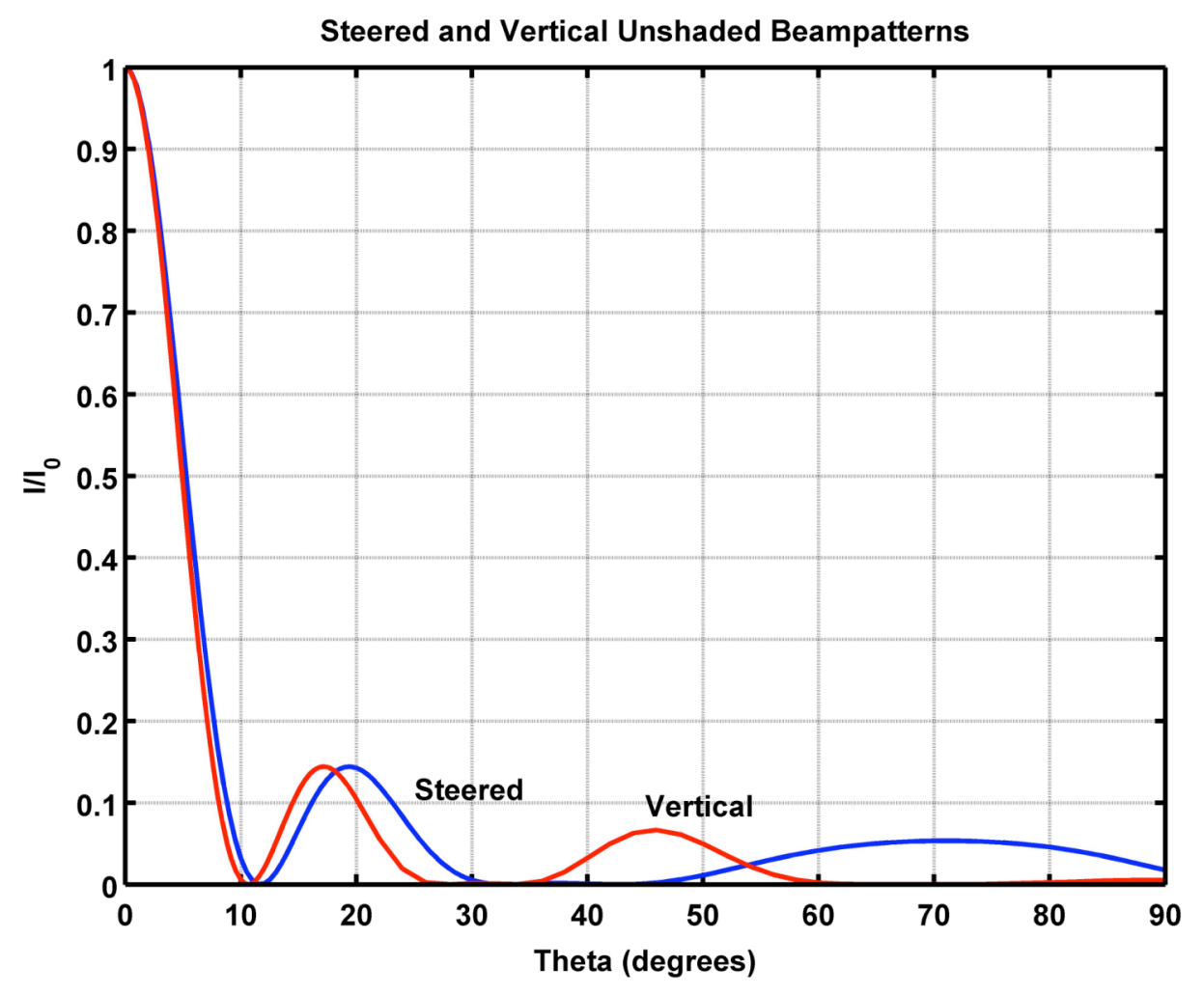

Figure 2.4 A comparison of steered and vertical beampatterns for the unshaded case. Beampatterns are plotted as normalized two-way intensity.

The vertical and steered beampatterns have very similar main lobes. Table 2.1

summarizes the characteristics of the unshaded steered and vertical beams (the $19^{\circ}$ beam characteristics are given relative to the mainlobe).

\begin{tabular}{|l|c|c|}
\hline \multicolumn{1}{|c|}{ Beam } & $\mathbf{1 9}^{\circ}$ & $\mathbf{0}^{\circ}$ \\
\hline Location of Sidelobes & $-71.4^{\circ},-19.6^{\circ}, 19.6^{\circ}, 71.4^{\circ}$ & $-46^{\circ},-17.2^{\circ}, 17.2^{\circ}, 46^{\circ}$ \\
\hline Location of Minima & $-39.3^{\circ},-11.9^{\circ}, 11.9^{\circ}, 39.3^{\circ}$ & $-28^{\circ},-10.8^{\circ}, 10.8^{\circ}, 28^{\circ}$ \\
\hline Relative intensity at $\mathbf{9 0}^{\circ}$ & $1.315 \times 10^{-2}$ & $5.373 \times 10^{-3}$ \\
\hline 3dB Width & $10^{\circ}$ & $9.6^{\circ}$ \\
\hline 6dB Width & $14^{\circ}$ & $12.8^{\circ}$ \\
\hline
\end{tabular}

Table 2.1 Characteristics of unshaded $19^{\circ}$ and $0^{\circ}$ beampatterns.

To reduce the sidelobe amplitude, the SFAS can also beamform using shading. A comparison of the normalized 2-way intensity for the 1-dimensional shaded and unshaded vertical beam is shown in Figure 2.5. Again, only positive angles are shown. 
Note that shading decreases the intensity of the sidelobes, while widening the main lobe. It is also noteworthy that the shaded beampattern's intensity is about a factor of 5 higher than the unshaded beampattern's intensity at 90 degrees.

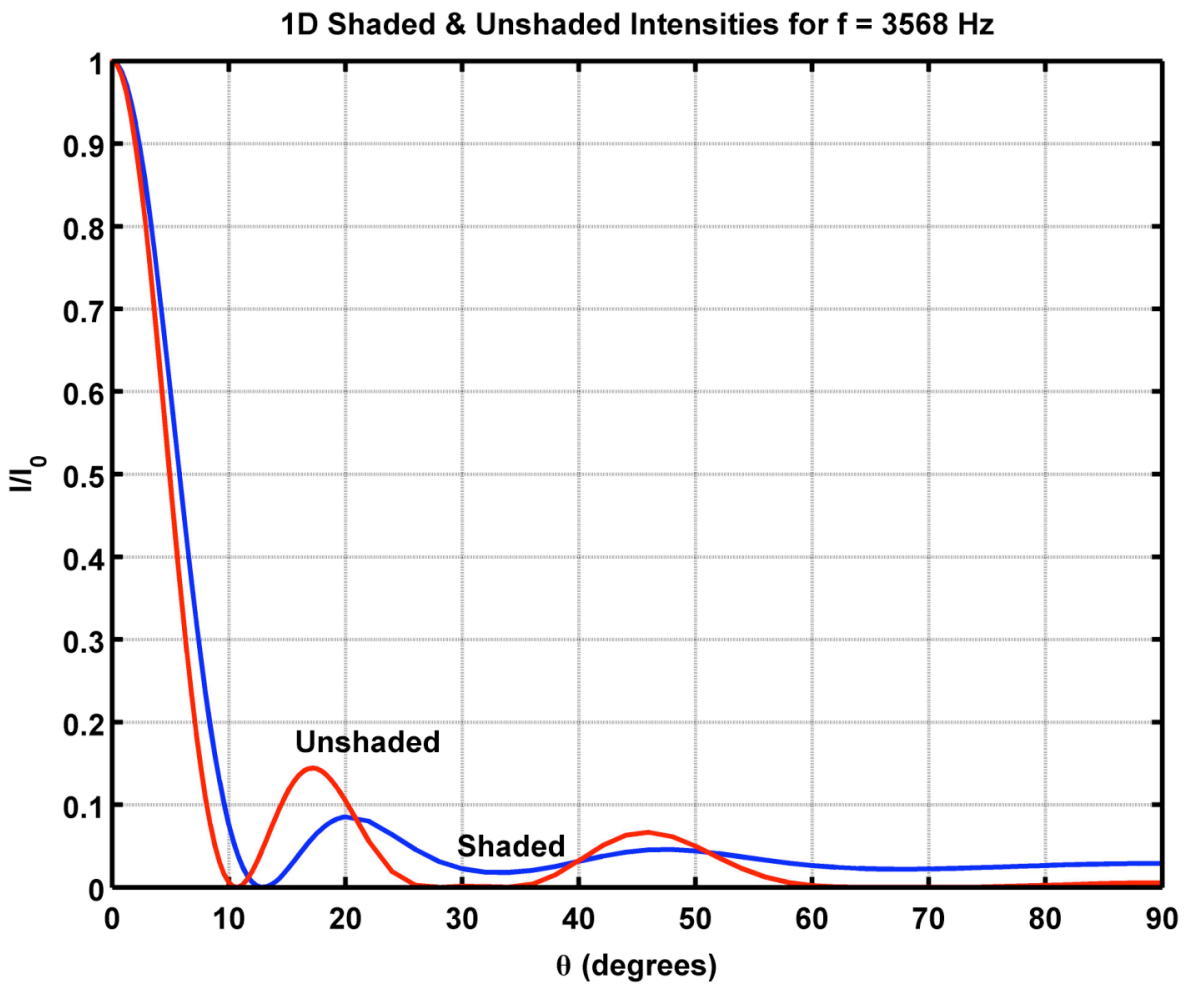

Figure 2.5 Scintec 1-dimensional beampattern for the shaded and unshaded vertical beampattern. Intensity is two-way and normalized.

The important characteristics of the 1-dimensional shaded and unshaded vertical beampatterns are summarized in Table 2.2.

\begin{tabular}{|l|c|c|}
\cline { 2 - 3 } \multicolumn{1}{c|}{} & Shaded Beampattern & Unshaded Beampattern \\
\hline Location of Sidelobes & $-48^{\circ},-20^{\circ}, 20^{\circ}, 48^{\circ}$ & $-46^{\circ},-17.2^{\circ}, 17.2^{\circ}, 46^{\circ}$ \\
\hline Location of Minima & $-34^{\circ},-12.8^{\circ}, 12.8^{\circ}, 34^{\circ}$ & $-28^{\circ},-10.8^{\circ}, 10.8^{\circ}, 28^{\circ}$ \\
\hline Relative intensity at $\mathbf{9 0}^{\circ}$ & 0.0290 & $5.373 \times 10^{-3}$ \\
\hline 3dB Width & $11.2^{\circ}$ & $9.6^{\circ}$ \\
\hline 6dB Width & $15.2^{\circ}$ & $12.8^{\circ}$ \\
\hline
\end{tabular}

Table 2.2 Summary of Scintec vertical beampattern characteristics. 
The normalized two-way intensity as a function of $(x, y)=(k \Delta / \pi) \sin \theta(\cos \phi, \sin \phi)$ for the 2-dimensional shaded SFAS vertical beampattern is shown in Figure 2.6. Here $\Delta=$ $0.05 \mathrm{~m}$ is the transducer spacing in both $\mathrm{x}$ and $\mathrm{y}$. Note that the central lobe is approximately azimuthally symmetric.

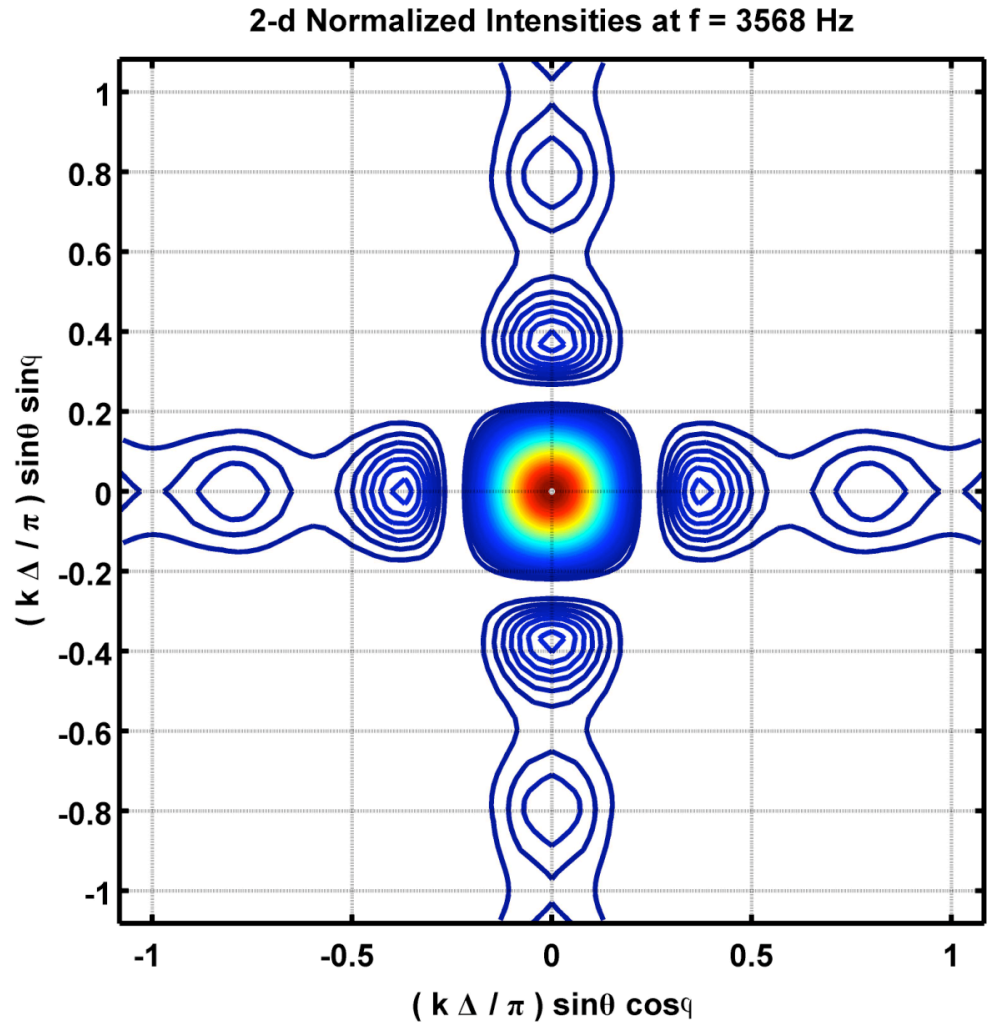

Figure 2.6 2-dimensional normalized intensities for the shaded SFAS vertical beampattern at $3568 \mathrm{~Hz}(\lambda \sim 0.1 \mathrm{~m})$. 


\section{Chapter 3}

\section{Sodar Platforms}

\subsection{Overview}

Since buoy motion decreases SNR by reducing the overlap between the transmit $(\mathrm{T})$ and receive $(\mathrm{R})$ beams, it is important to understand how the shape of a buoy affects its response to wave forcing.

We want to choose a buoy that does not experience a significant amount of rotational motion (i.e. pitch, roll, and yaw). Tilts in pitch and roll will likely have a negative effect on sodar performance since the main lobe of the sodar's beampattern has a 6-dB width of only $10^{\circ}$. Hence buoy tilts in pitch and roll need to be small compared to the width of the main lobe in order for the sodar to get a strong return. This is a stiff requirement since many oceanographic buoys often tilt in excess of $10^{\circ}$ due to wave forcing. In contrast, their heading changes typically are slow, so that rotational motion in yaw is expected to have a smaller impact on sodar performance than pitch and roll.

Translational motion (heave, surge, and sway) will also have a negative effect on sodar performance, but to a lesser degree than rotational motions. Sodar range bins are typically 5-10 m, so buoy motions in heave, for example, are small compared to sodar range resolution. Therefore, there is not as strict a requirement on buoy heave.

At one end of the spectrum of oceanographic buoys there are large spar buoys, such as the $110 \mathrm{~m}$ long "Floating Instrument Platform" (FLIP), operated by the Scripps Institution of Oceanography, which does not respond to wave forcing (http://sio.ucsd.edu/voyager/flip/index.html). FLIP is stable in waves because its resonant frequency is below the wave band. The following example explains why spar buoys are stable. A cylinder of length, $L$, floating upright has a resonant period of $2 \pi \sqrt{L / g}$. Hence, a simple spar greater than $25 \mathrm{~m}$ in length will be insensitive to $10 \mathrm{~s}$ waves, and a spar buoy, such as FLIP, that is greater than $100 \mathrm{~m}$ long will show no response to $20 \mathrm{~s}$ waves. Spar buoys also need freeboard so that they are not overtopped by passing waves. A large spar would be a nice platform to host a sodar if one is looking to make 
measurements far offshore. From the point of view of wind turbine site characterization and coastal atmospheric physics, however, a large spar buoy will not fit the bill. A buoy that is not portable and clearly cannot be deployed in coastal waters, will not meet our needs since we require a platform that can easily be deployed in a variety of locations, including littoral areas.

In contrast to spar buoys is the discus buoy, which follows the ocean surface in pitch, roll, and heave. These buoys also tend to follow the surface in surge and sway, depending on the mooring arrangement. An example of a discus buoy is the three-meter discus buoy used by the National Data Buoy Center (NDBC) to measure waves. Since the 3-meter discus buoy is so widely used in Oceanographic research, it is of interest to evaluate its suitability as a possible platform in comparison to other platforms. We do this in Section 3.2, below.

A buoy design that shares the characteristics of a stable spar buoy as well as a discus buoy is a hybrid spar buoy called the "Air Sea Interaction Spar" (ASIS), shown schematically in Figure 3.4. ASIS is a short spar with a heavily damped resonance. As a result, its response to waves resembles a low-pass filter with a cutoff frequency ranging from 6 to 8 seconds, depending on its particular configuration. The ASIS buoy is part of a system that includes a $50 \mathrm{~m}$ floating tether that connects it to a surface mooring. This allows ASIS to follow wave motion relatively freely in heave, surge and sway. However, ASIS tilts in pitch and roll generally are small. ASIS is potentially a good platform to host a sodar, so we will discuss its design in more detail in Section 3.3.

\subsection{Three meter discus buoy characteristics}

Since sodar works by measuring the Doppler shift, the rms error in the wind velocity measurement is affected by the motion of the instrument. In this section, we investigate the rms tilts of a 3-meter discus buoy. A discus buoy 3 meters long follows waves roughly up to twice its diameter (6 meters). This corresponds to a wavenumber, $k=1 \mathrm{~m}^{-1}$. With $k$ we can solve the dispersion relation for deep water, $\omega=\sqrt{g k}$, to get the maximum resolvable frequency seen by the buoy. In this case, $\omega=2 \pi f=3.3 \mathrm{rad} / \mathrm{s}$, so 
a 3-m discus buoy ideally will follow the surface slope of waves up to frequencies $(f)$ of $0.5 \mathrm{~Hz}$.

To get an understanding of how the buoy behaves in a variety of different conditions, we look at probability distributions of buoy $r m s$ tilts for 2005. To predict the $r m s$ tilts of the discus buoy, we apply a transfer function to a year's worth (2005) of Martha's Vineyard Coastal Observatory (MVCO) wave slope spectra. Our transfer function has an ideal low-pass filter form that is unity from 0 to $0.5 \mathrm{~Hz}$, and vanishes at higher frequencies. A plot of the probability distribution of $r m s$ tilts computed over 20 minute bursts for the year 2005 is given in Figure 3.1

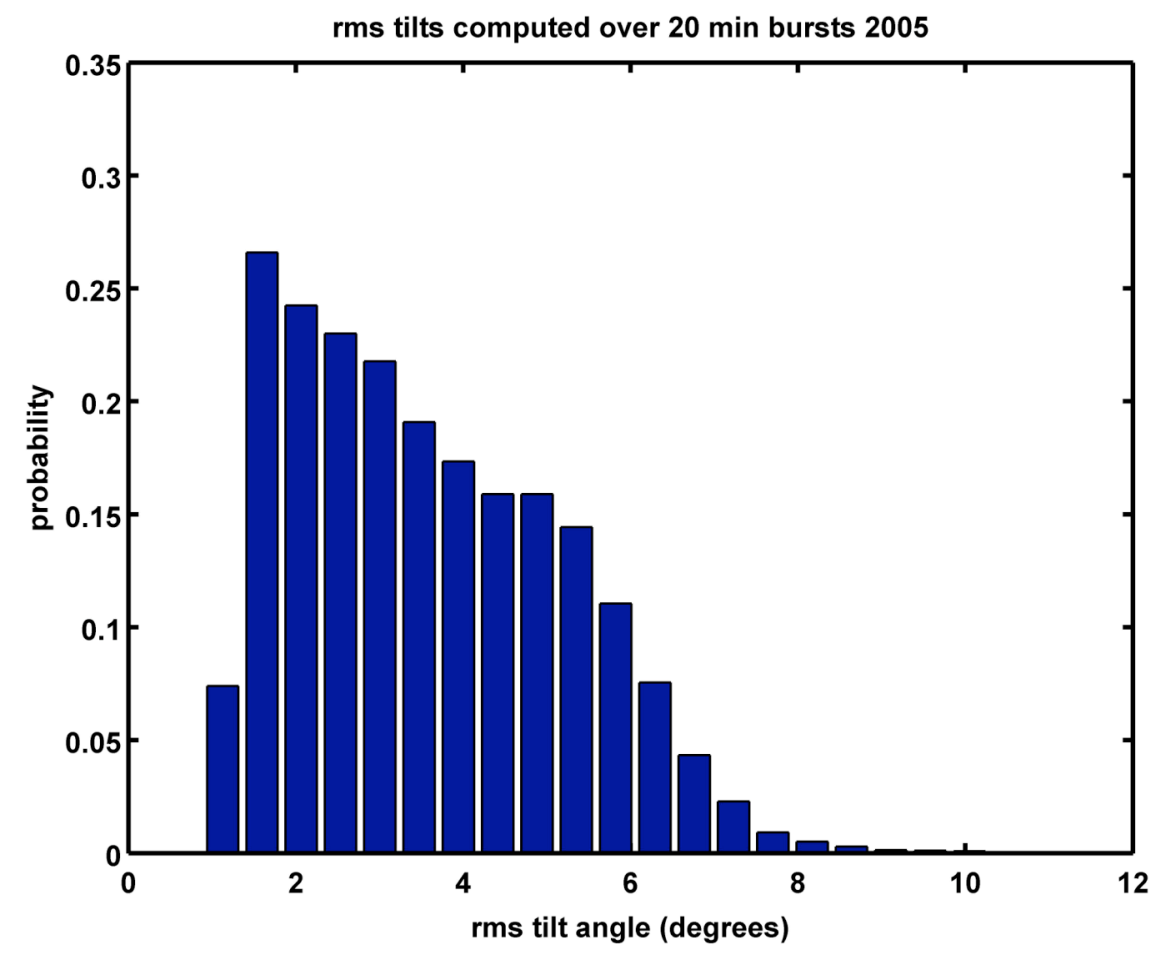

Figure 3.1 Distribution of 3-meter discus buoy rms tilts for 2005.

Reductions in SNR due to three meter discus buoy tilts can be considerable, so we want to get an idea of how other buoys respond under the same conditions. To do this we compare the performance of the three meter discus buoy to the performance of an Air-Sea Interaction (ASIS) Spar for the same days of data. We have six days of ASIS motion 
data from the ASIS that we deployed south of the MVCO tower in the fall of 2005 (the details of this experiment are described in Appendix B, while the calibrations of the sensors used in the experiment can be found in Appendix D). In figure 3.2, we plot a comparison of measured ASIS rms tilts to 3-meter discus buoy tilts (for the same days that we have ASIS data), predicted from MVCO measurements. On average, 3-meter discus buoy rms tilts are roughly 4 times greater than ASIS tilt. Since ASIS buoy tilts are much smaller, we continue an investigation into the characteristics of the ASIS platform.

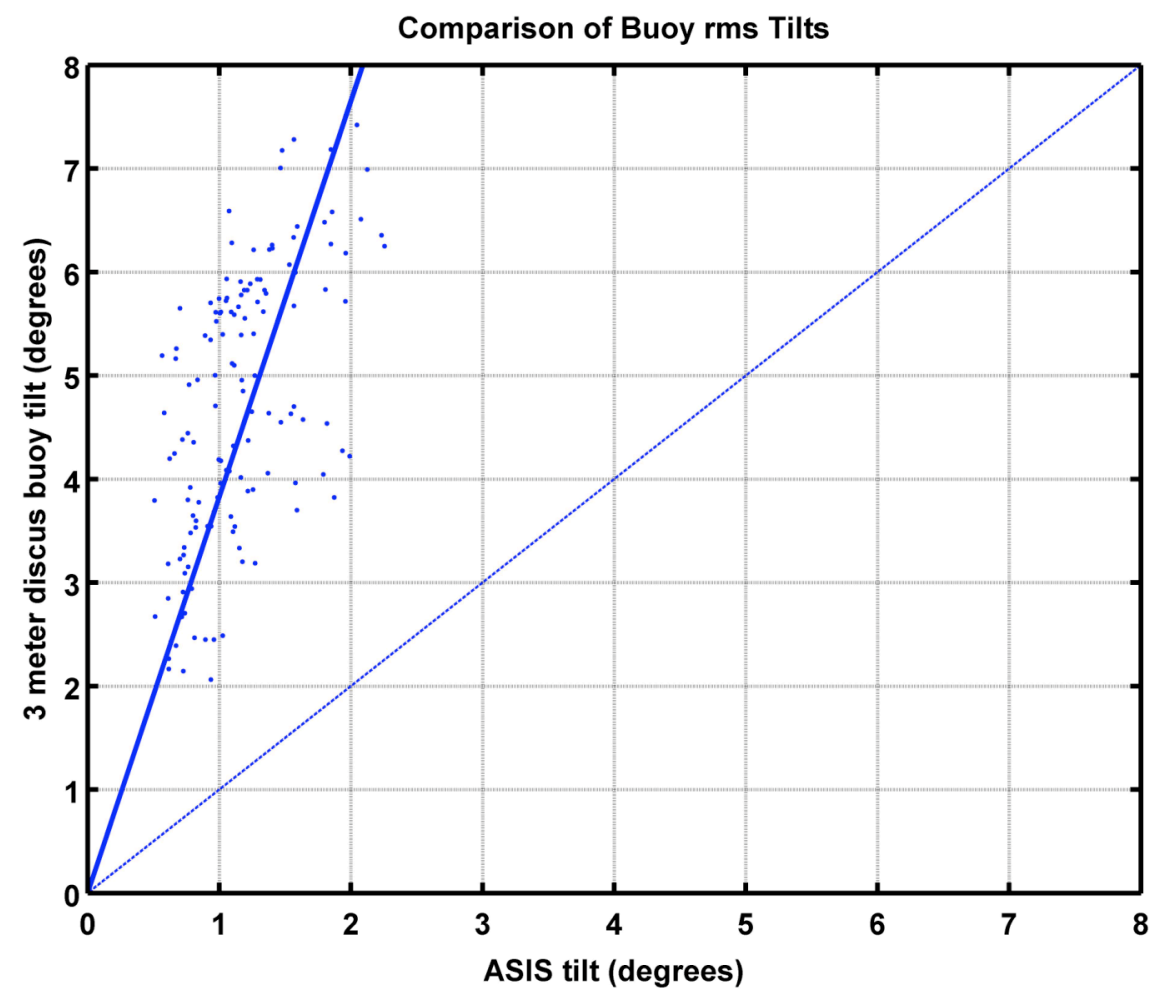

Figure 3.2 A comparison of 3-meter discus buoy predicted rms tilts to ASIS measured rms tilts. The solid line shows a linear least squares fit to the data and has a slope of 3.8.

\subsection{ASIS design characteristics}

In this section, we discuss the general design principles underlying ASIS, and sketch a typical buoy design and instrumentation payload to support sodar measurements. ASIS (Graber et.al., 2000) is a short, highly damped spar buoy (Figure 3.3). Unlike a true spar buoy, ASIS moves with long waves (i.e. periods $>7-8 \mathrm{~s}$ ) but is relatively insensitive to 
shorter wavelengths. Because it has a small response to pitch and roll, it likely is a good platform for air-sea measurements. ASIS has the added capability of being able to be disassembled and packed in a 20 -foot shipping container. It is also advantageous in that it can be deployed from small research vessels.

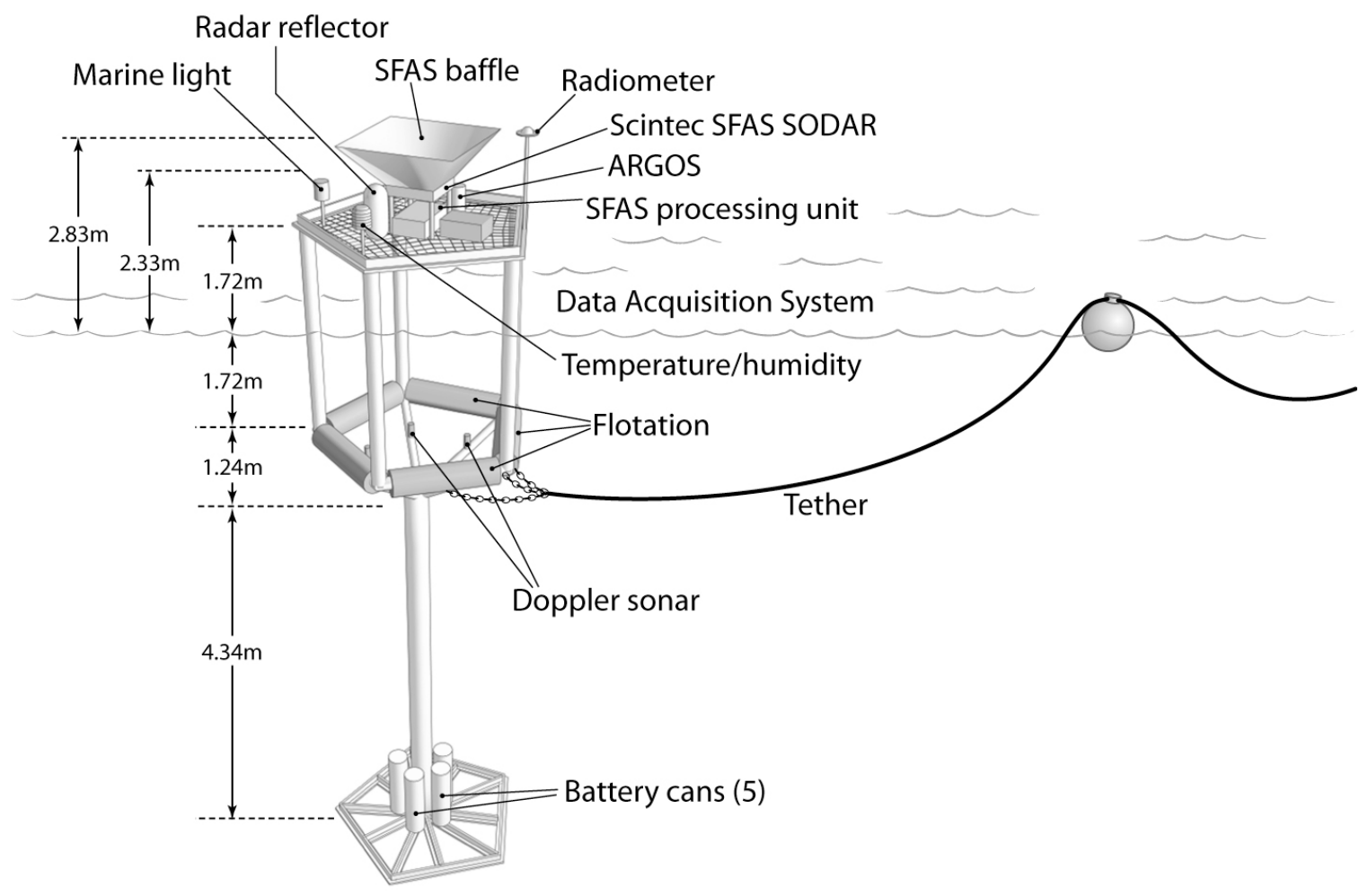

\section{Figure 3.3 Drawing of a typical ASIS buoy.}

ASIS is designed to follow long waves and be stable in short waves. Hence, the dynamic range of perceived wave motion by the buoy is reduced, thus minimizing the risk of overtopping in the presence of long waves with larger amplitudes. Because the buoy has considerable draft, it can be ballasted to be stable in pitch and roll in typical New England sea states. The buoy's buoyancy is primarily provided by the five upright columns and the five floats (horizontal cylinders) that are situated at the base of the columns. The main tube is free-flooding and houses adjustable ballast for trim. ASIS is typically ballasted so that the mean water level is in the middle of the five uprights. The foot of the buoy is made up of expanded aluminum grating to increase drag in heave. 
Battery cans are located at the foot and add a considerable amount of ballast, which helps to keep the buoy stable.

Although ASIS can be used as a freely drifting surface platform, it is more useful in our application as a moored buoy. Since the five uprights do not provide much reserve buoyancy (they total $124 \mathrm{lbs} / \mathrm{ft}$ of immersion), ASIS must be tethered using a floating line to a nearby surface mooring in order to prevent large vertical forces on the buoy. The ASIS buoy, tether, and surface mooring make up the ASIS "system".

The dimensions of a typical ASIS buoy are shown in Table 3.1. The corresponding air weights and buoyancies are shown in Table 3.2. The draft of ASIS can adjusted by adding or removing weights from the main tube.

\begin{tabular}{|c|c|c|c|}
\hline Item & Quantity & Shape & Dimensions (LxD) \\
\hline & & & (inches) \\
\hline Foot & 1 & Pentagon & $4.2 \times 39$ \\
\hline Batteries & 5 & Cylinder & $38 \times 12.7$ \\
\hline Main Tube & 1 & Cylinder & $170 \times 12$ \\
\hline Candelabra tubes & 5 & Cylinder & $53 \times 4$ \\
\hline Floats & 5 & Cylinder & $44 \times 12.7$ \\
\hline Uprights & 5 & Cylinder & $137 \times 8.4$ \\
\hline Top Plate & 1 & Pentagon & $4.2 \times 49$ \\
\hline
\end{tabular}

Table 3.1 Dimensions of ASIS principal structural components.

The dimensions of ASIS can be varied somewhat in order to fine-tune its response to different sea states. The response functions of ASIS generally have the shape of a low pass filter. The drag due to the expanded metal in the foot damps the resonant heave response of the buoy (Graber et. al., 2000). 


\begin{tabular}{|c|c|c|c|c|}
\hline Item & Details & Quantity & Weight in Air & Buoyancy \\
\hline & & & (pounds) & (pounds) \\
\hline \multirow[t]{8}{*}{ ASIS Buoy } & Foot & 1 & 118.9 & 44.8 \\
\hline & Batteries & 5 & 1115 & 887.5 \\
\hline & Main Tube & 1 & 241.5 & 91 \\
\hline & Candelabra tubes & 5 & 69 & 123 \\
\hline & Floats & 5 & 136 & 1027 \\
\hline & Uprights & 5 & 634.5 & 700.5 \\
\hline & Top Plate & 1 & 266.1 & \\
\hline & Ballast & & 114.8 & \\
\hline \multirow[t]{10}{*}{ Instrumentation } & SFAS SODAR & 1 & 46 & \\
\hline & SFAS table & 1 & 36 & \\
\hline & SFAS processing unit & 1 & 38 & \\
\hline & SFAS baffle & 1 & 20 & \\
\hline & Data Acquisition Box & 1 & 21 & \\
\hline & ARGOS & 1 & 5 & \\
\hline & Freewave modem & 1 & 2 & \\
\hline & Marine Light & 1 & 5 & \\
\hline & Radar Reflector & 1 & 5 & \\
\hline & Total Weight & & 2874 & 2874 \\
\hline
\end{tabular}

Table 3.2 Weights and buoyancy of ASIS structural components and sensors.

\subsection{ASIS instrumentation and data acquisition}

In addition to wind profiling, ASIS is capable of making other measurements. These measurements can be divided into three categories: meteorological, buoy motion, and waves.

Meteorological measurements of interest are temperature, relative humidity, solar radiation, barometric pressure and rainfall. Eppley Labs and RM Young manufacture many of the instruments capable of these measurements. A typical list of instrumentation is given in Table 3.3. Data can be digitized and recorded with the buoy's main data acquisition system or a separate data logger such as a Campbell CR-10X logger.

The buoy's motion is measured with an inertial motion-sensing unit (IMU). The IMU consists of three orthogonal accelerometers and three orthogonal rate gyros. A compass must also be included to measure buoy heading. BEI Systron Donner makes a commonly used IMU (see Table 3.3). Since accelerometers cannot distinguish between tilt and lateral acceleration, rate gyros must be used to determine tilt in the band of wave 
frequencies. Rate gyros measure angular velocity about an axis and must be integrated to obtain tilt. At frequencies well below the wave band, lateral accelerations are negligible, so accelerometers can be used to measure tilt. Accelerometer and rate gyro data can be combined using a complementary filter to obtain acceleration and tilt over the full range of frequencies (Brown and Hwang,1997; Graber et. al., 2000).

Waves can be measured from the ASIS buoy using a six-element array of capacitance wave staffs. Wave staffs are positioned on the faces of each of the five uprights as well as at the center of the pentagonal cage. An array of this type can be purchased from RBR Ltd. for $\$ 7350$. However, these waves staffs are not robust enough for long deployments. A new method of making wave measurements from ASIS is currently under development at WHOI and consists of a similar array of upward-looking $2 \mathrm{MHz}$ Doppler sonars. These sonars determine the range to the surface by emitting sound pulses and measuring the time of flight.

\begin{tabular}{|c|c|c|}
\hline Item & Cost (\$) & Manufacturer \\
\hline ASIS buoy & 35,000 & WHOI \\
\hline Surface mooring and tether & 10,000 & WHOI \\
\hline D-cell batteries (60 kW-h) & 4,000 & Matthews Assoc. \\
\hline SFAS SODAR & 65,000 & Scintec \\
\hline Inertial motion unit & 15,000 & BEI Systron Donner \\
\hline Compass & 1,600 & PNI Corporation \\
\hline Data acquisition system & 5,000 & WHOI \\
\hline Radar reflector \& Marine light & 1,000 & Carmanah \\
\hline ARGOS & 1,000 & Seimac Corp. \\
\hline Freewave modem (2) & 2,000 & Freewave Technologies \\
\hline Rain gauge & 600 & RM Young \\
\hline Temperature and $\mathrm{RH}$ probe & 900 & RM Young \\
\hline Precision spectral pyranometer & 2,200 & Eppley Lab \\
\hline Precision infrared radiometer & 3,300 & Eppley Lab \\
\hline Doppler sonar wave gauge (6) & 15,000 & WHOI \\
\hline Acoustic Doppler Current Profiler & 25,000 & RD Instruments \\
\hline Total: & 186,600 & \\
\hline
\end{tabular}

Table 3.3 Typical ASIS sensors and cost. 


\section{Chapter 4}

\section{Analysis of ASIS Platform Dynamics}

\subsection{Overview}

In this chapter we model ASIS platform dynamics and analyze ASIS's measured response to different sea states. We would like to get an idea of how ASIS responds in a variety of conditions, so that we can ultimately predict and understand how best to deploy a sodar. To get a good overview of ASIS response we look at a year's worth of data along the continental shelf from New York and New England. We use the data to perform a statistical analysis of buoy response to waves and wind. With this data, we can also verify our ASIS models that were derived from theory. A better understanding of ASIS platform dynamics provides the knowledge required to successfully deploy a sodar.

\subsection{Wind and wave conditions}

Wind and wave conditions used in this analysis are chosen based on MVCO and National Data Buoy Center (NDBC) data. A map of the NDBC buoys in the Northeast region is shown in Figure 4.1. The buoys selected to provide data for our models are shown in Table 4.1.

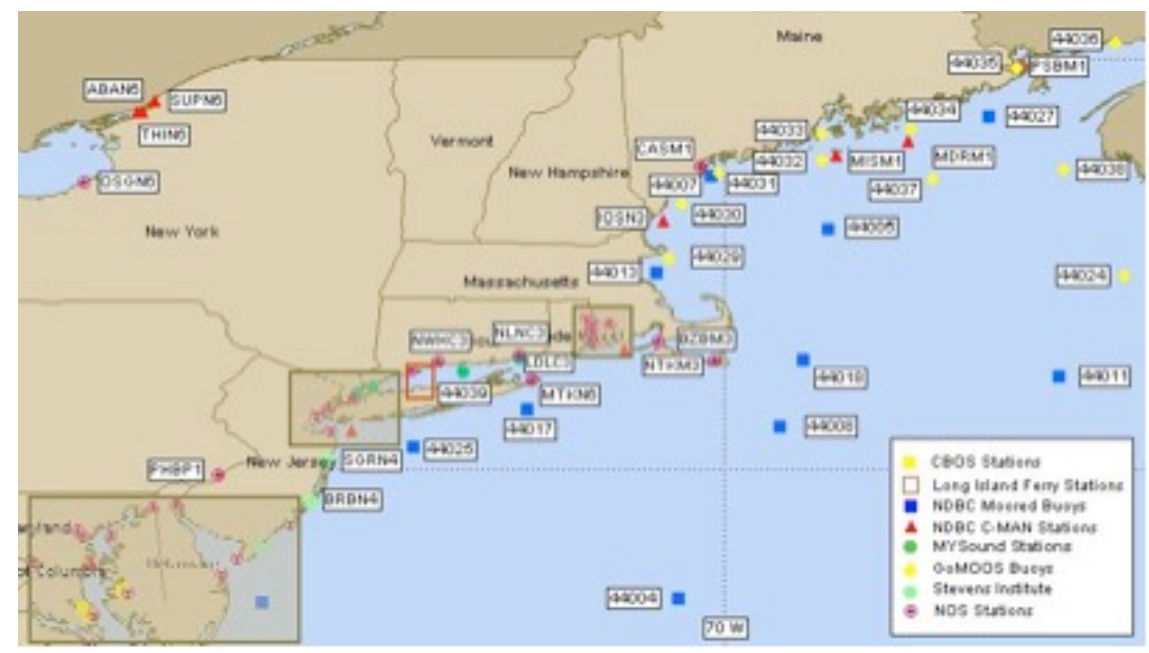

Figure 4.1 NDBC Northeastern buoys. 


\begin{tabular}{|c|c|c|l|}
\hline Buoy & Latitude & Longitude & \multicolumn{1}{c|}{ Location } \\
\hline 44005 & $40^{\circ} 11^{\prime} 12^{\prime \prime} \mathrm{N}$ & $69^{\circ} 09^{\prime} 48^{\prime \prime} \mathrm{W}$ & 78nm East of Portsmouth, ME \\
\hline 44008 & $40^{\circ} 30^{\prime} 00^{\prime \prime} \mathrm{N}$ & $69^{\circ} 25^{\prime} 53^{\prime \prime} \mathrm{W}$ & 54nm Southeast of Nantucket, MA \\
\hline 44017 & $40^{\circ} 41^{\prime} 32^{\prime \prime} \mathrm{N}$ & $72^{\circ} 02^{\prime} 52^{\prime \prime} \mathrm{W}$ & 23nm Southwest of Montauk Point, NY \\
\hline 44018 & $41^{\circ} 15^{\prime} 30^{\prime \prime} \mathrm{N}$ & $69^{\circ} 17^{\prime} 40^{\prime \prime} \mathrm{W}$ & 30nm East of Nantucket, MA \\
\hline 44025 & $40^{\circ} 15^{\prime} 01^{\prime \prime} \mathrm{N}$ & $73^{\circ} 09^{\prime} 59^{\prime \prime} \mathrm{W}$ & 33nm South of Islip, NY \\
\hline
\end{tabular}

Table 4.1 Selected NDBC buoys and their locations.

NDBC buoys 44025 and 44017 are located close to Long Island while buoys 44008 and 44018 are in deeper water southeast of Cape Cod. Buoy 44005 is located near the Gulf of Maine. All buoys recorded wind speed and direction as well as scalar wave properties. However, only buoy 44025 recorded wave direction.

A statistical analysis of the wind and waves at these buoys, and at MVCO, show that they all experienced similar conditions. Figures 4.2 and 4.3 present a typical comparison by showing histograms of wind speed and direction, significant wave height and mean wave periods and direction (where available) for NDBC buoys 44025 (off Long Island) and 44018 (southeast of Cape Cod).

Therefore, in the following discussion, in order to increase the sampling statistics, we will combine the data from the four NDBC buoys listed above. Histograms of the combined data set (from all 4 buoys) are shown in Figure 4.4. Winds are seen to be predominately from the southwest with a mode of $5 \mathrm{~m} / \mathrm{s}$. Waves are predominately from the southeast, with a mode of significant height and peak period of about 1 meter and 7 seconds, respectively. 

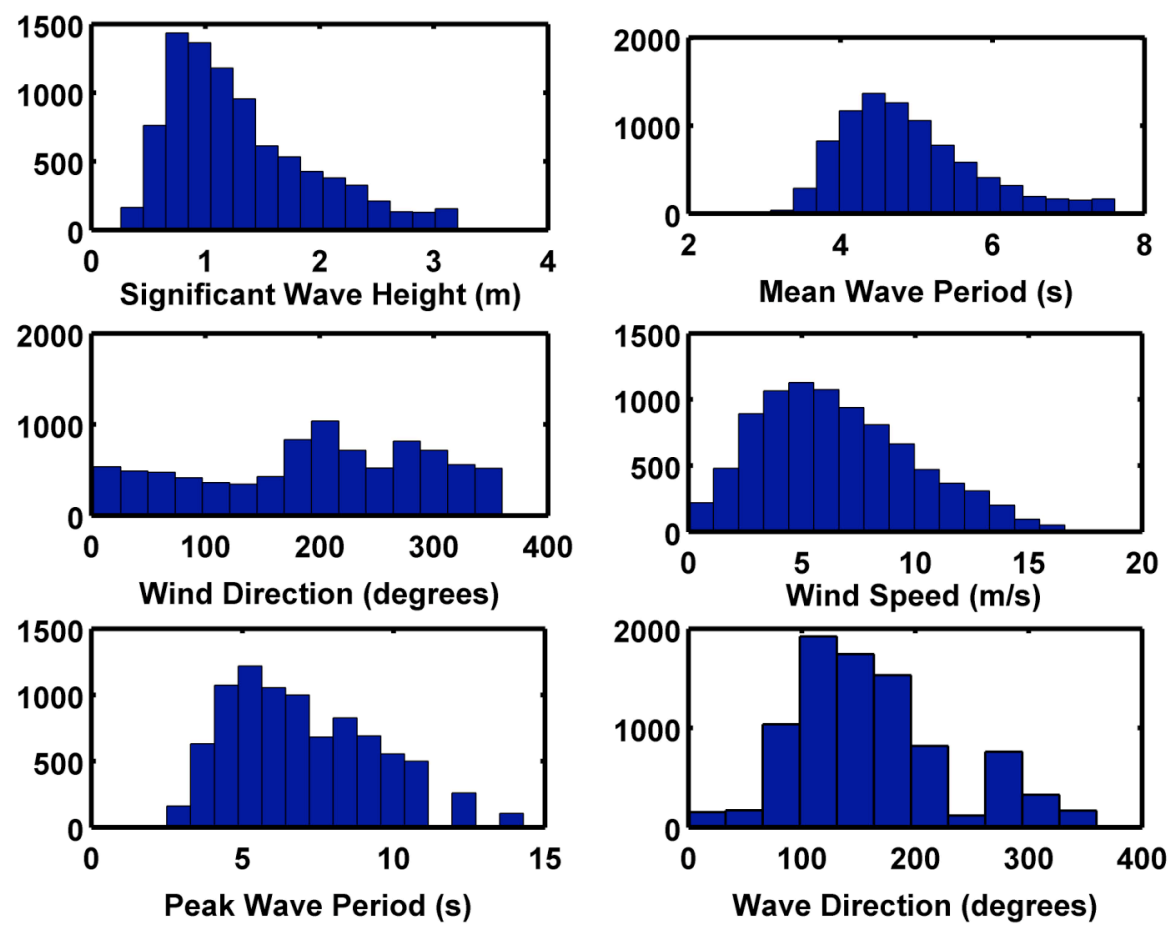

Figure 4.2 Histograms of wind and wave conditions during 2004 by NDBC buoy 44025, located off Long Island, NY. 

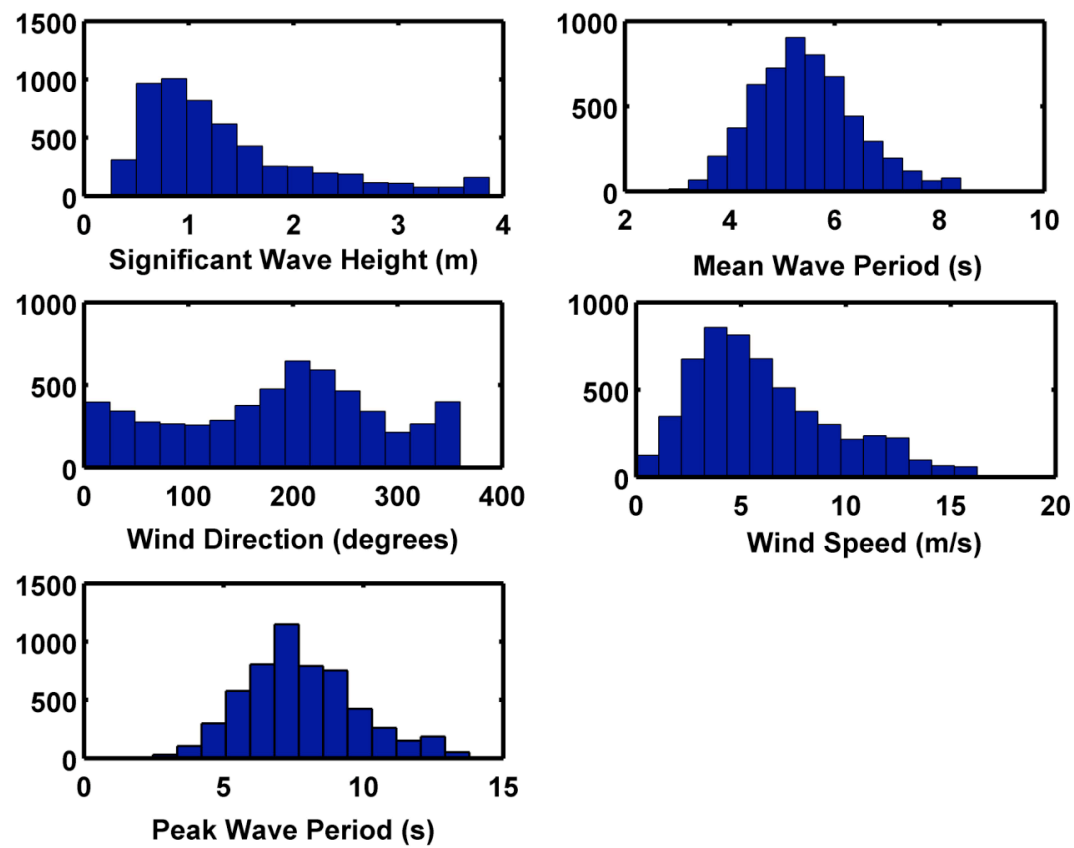

Figure 4.3 Histograms of wind and wave conditions in 2005 by NDBC buoy 44018, located southeast of Cape Cod, MA.
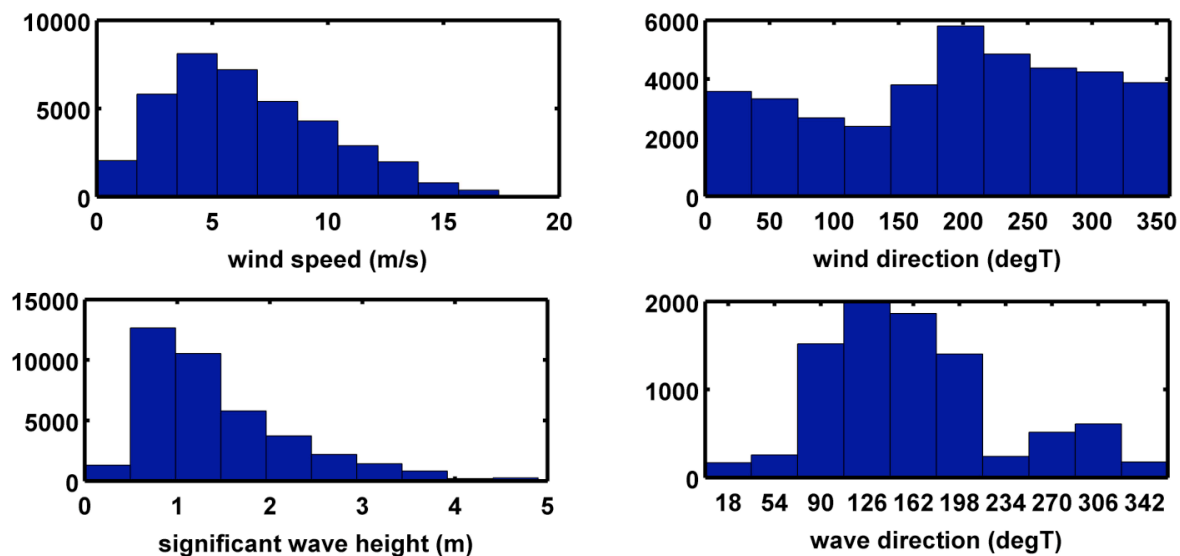
wave direction (degT)
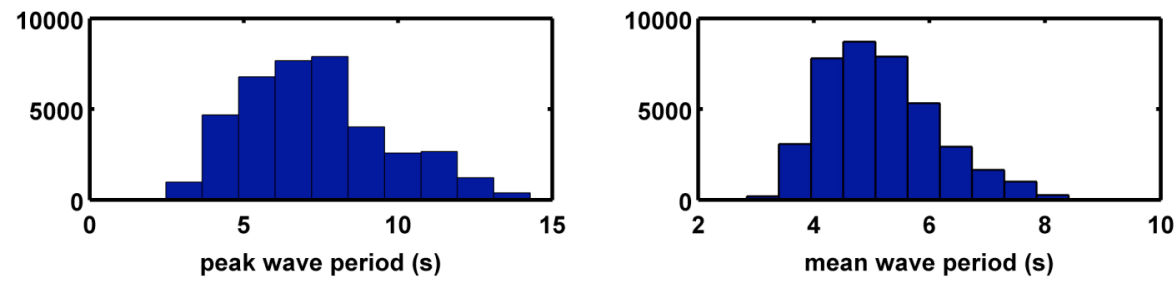

Figure 4.4 Histograms of combined data from all five NDBC buoys. 
From the combined NDBC data, we define three sea state conditions as: "typical", "high" and "extreme". "Typical" conditions are taken to be the mean wind speed of all winds exceeding a threshold of $3.5 \mathrm{~m} / \mathrm{s}$. "High" winds are defined as the mean of the upper $5 \%$ of winds above $3.5 \mathrm{~m} / \mathrm{s}$. Wave periods and heights are taken to be averages over all wind events in each class. Our "extreme" condition will be defined later in terms of a rare storm event. Table 4.2 shows a listing of the characteristics of the "typical" and "high" classes defined from the combined 2004 NDBC data.

\begin{tabular}{|l|c|c|}
\hline Combined NDBC buoy data & "Typical" & "High" \\
\hline Wind speed $(\mathrm{m} / \mathrm{s})$ & 7.6 & 14.8 \\
\hline Dominant wave period $(\mathrm{s})$ & 5.0 & 7.9 \\
\hline Significant wave height $(\mathrm{m})$ & 1.6 & 3.3 \\
\hline Inverse wave age, $U_{10} / C_{p}$ & 1.0 & 1.2 \\
\hline
\end{tabular}

Table 4.2 Combined NDBC buoy data for typical and high classes.

The "inverse wave age" is defined to be the ratio of wind speed at 10 meters to the phase speed of the waves at the spectral peak and characterizes the state of development of the wave field.

\subsection{Measured ASIS response to wave forcing}

In this section we smooth and parameterize measured response functions in heave, pitch, roll and surge from an ASIS buoy. The response functions are described in Graber et. al (2000) and are for an ASIS similar to the buoy design described in this thesis. In fact, Graber et. al. (2000) measured response functions for two different ASIS buoys and showed that the configuration does not significantly effect the result. The empirical transfer functions are shown in Figures 4.5 - 4.8 (E. Terray, pers. comm.). Each figure shows the magnitude (top panel) and phase (bottom panel) of the response function (solid line), the wave forcing spectrum (dashed line - top panel) and the coherence (dashed line - bottom panel) between the measured quantity and the wave forcing. In the case of heave and surge, the forcing function is taken to be the wave height, whereas for buoy tilt it is taken to be the sea surface slope. The response functions shown are the average response over sea states with significant wave heights ranging from 0.5 to 3 meters. 

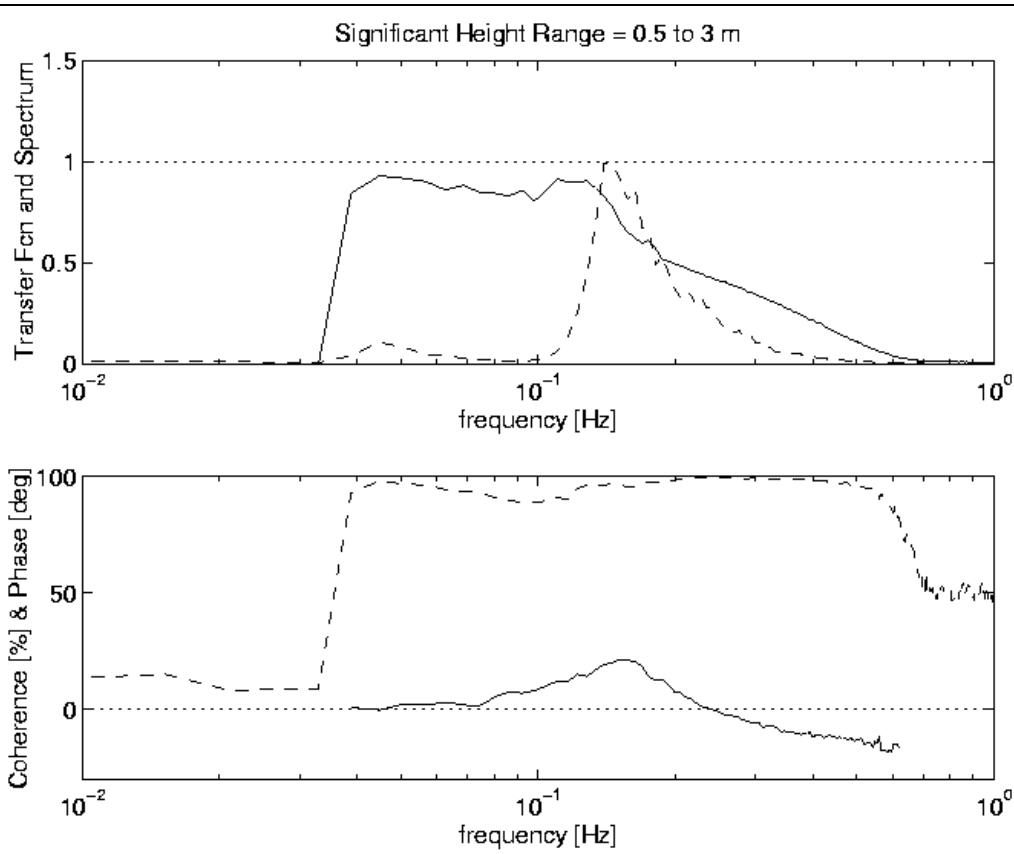

Figure 4.5 ASIS heave average response functions for sea states in the range of $\mathrm{Hs}=\mathbf{0 . 5}-3$ meters.

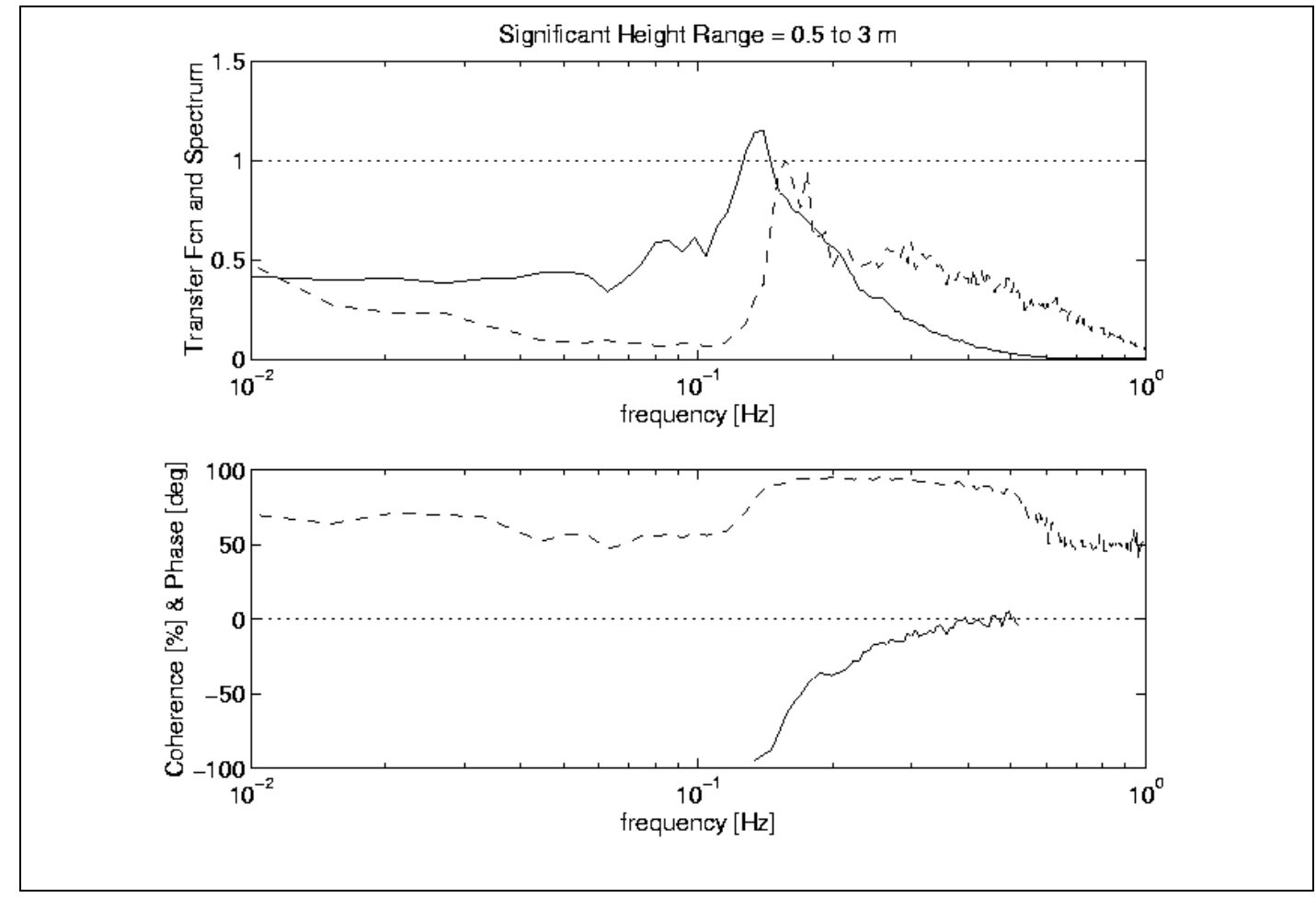

Figure 4.6 ASIS pitch average response functions for sea states in the range of

$$
\text { Hs }=0.5-3 \text { meters. }
$$




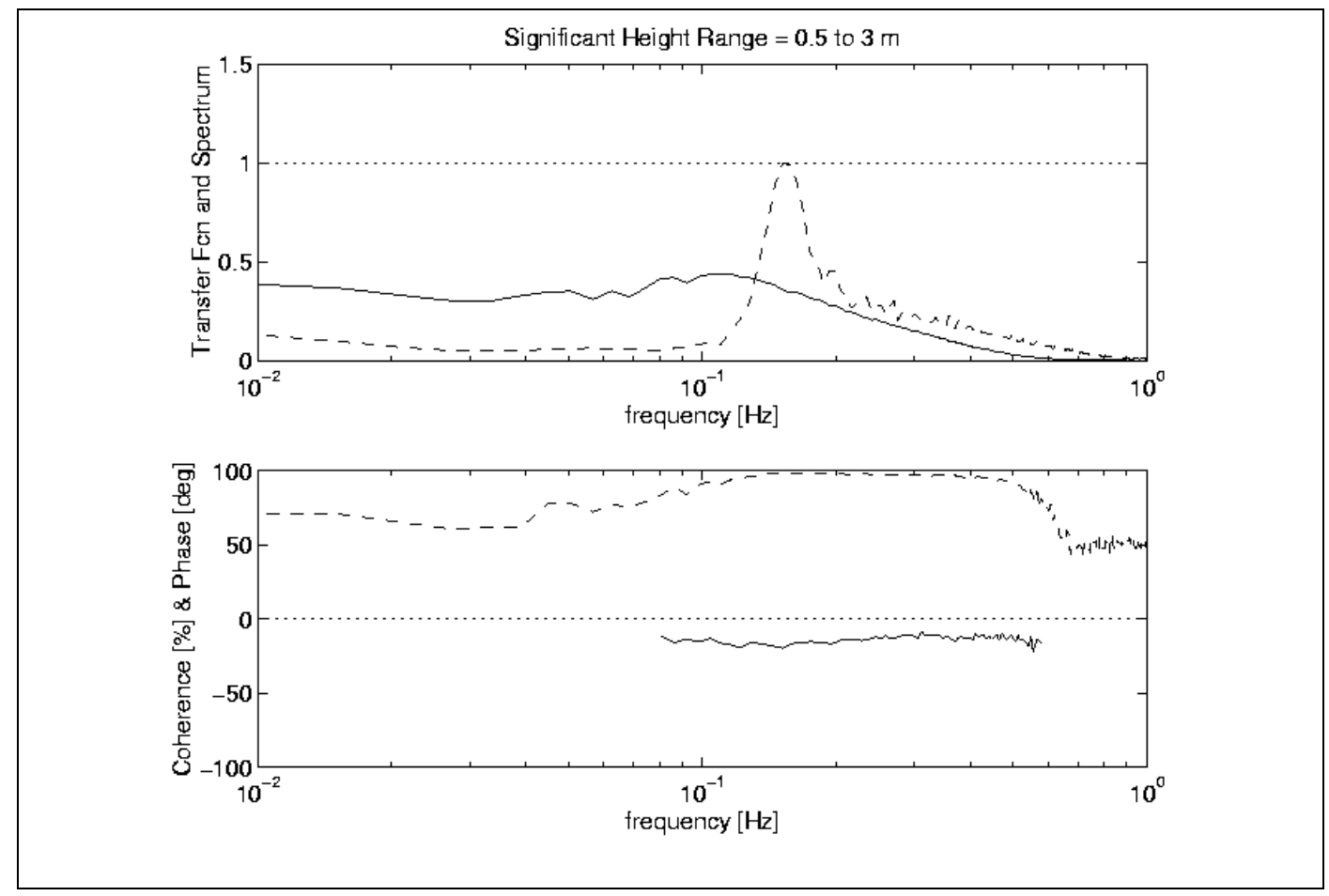

Figure 4.7 ASIS roll average response functions for sea states in the range of Hs $=0.5-3$ meters.

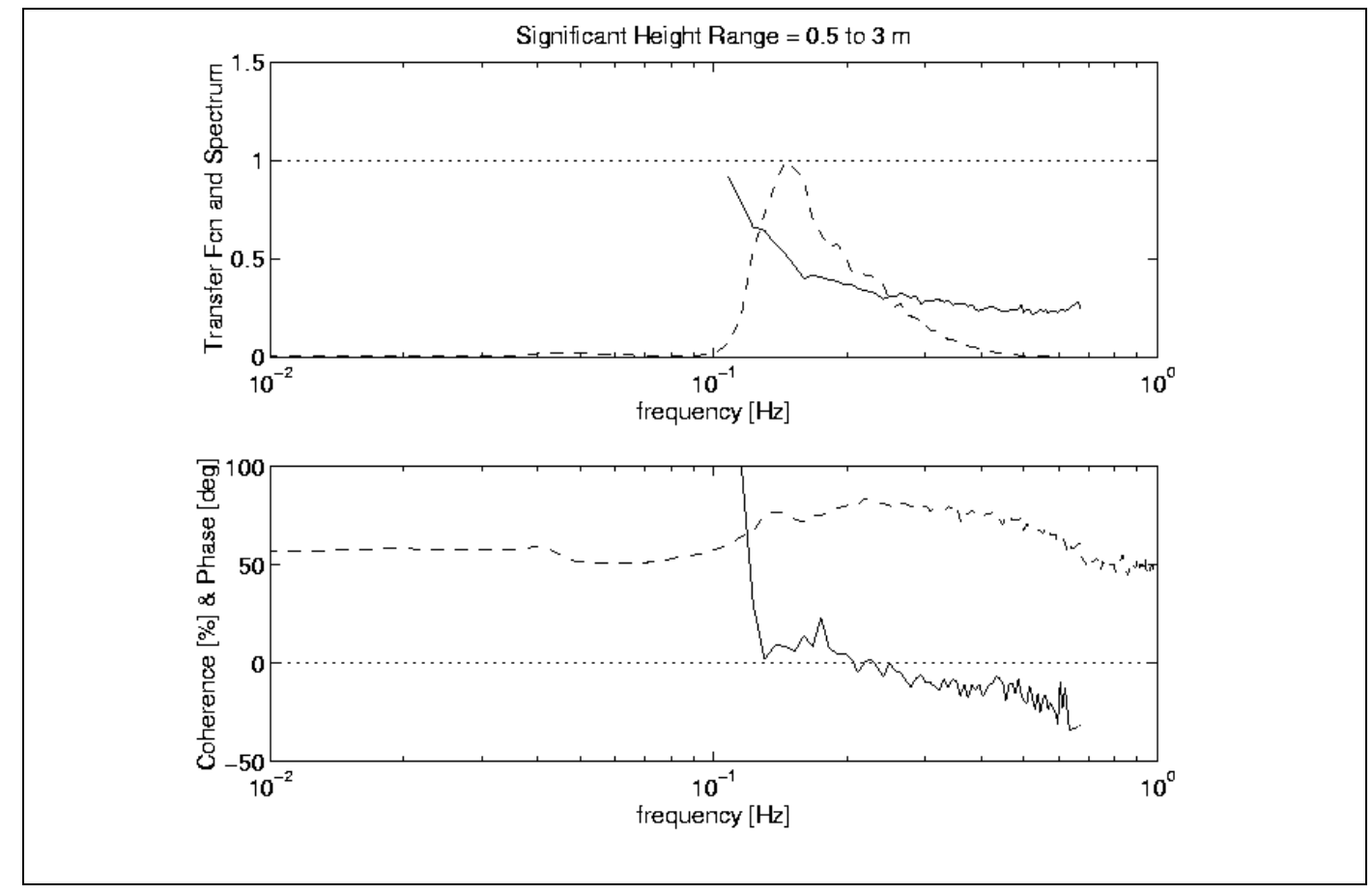

Figure 4.8 ASIS surge average response functions for sea states in the range of $\mathrm{Hs}=$ $0.5-3$ meters. 
We parameterized the empirical ASIS response functions by fitting each with a smoothing spline (see Figure 4.9). For the case of heave, pitch and surge, we continued the transfer functions to low frequency using the theoretical ideal response to long waves. So the phase of the heave response tends to zero and its magnitude is unity as $\mathrm{f} \rightarrow 0$. The phase of both pitch and surge tends to $\pi / 2$ (the difference in sign of the pitch is definitional) and the magnitude of the surge tends to unity. We have taken the magnitude of pitch to be 0.5 at low frequencies, in accord with the observations.

We have included the roll response for completeness. Although we expected the roll response to be closer to pitch than it is (since ASIS is a regular pentagon), it is quite different. The reason for this difference is not known.
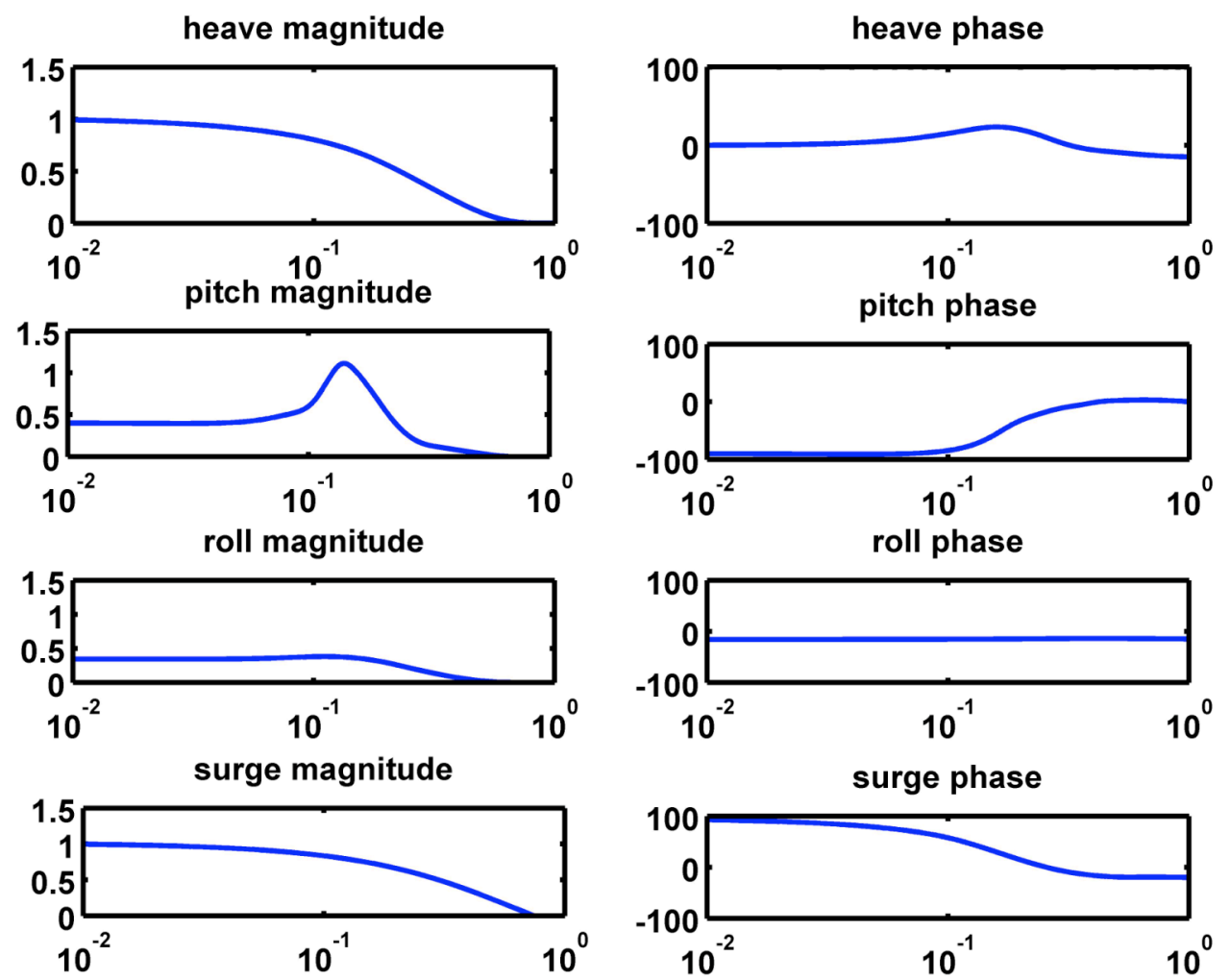

Figure 4.9 Parameterized ASIS response functions. 


\subsection{Comparison of the modeled and measured ASIS tilt response}

In this section we compare the parameterized ASIS response functions with data from our ASIS deployment. Since we did not measure waves from the buoy, the comparison must be statistical. We use wave height spectra measured at the MVCO 12-m node to estimate wave slope spectra for waves having frequencies below $0.5 \mathrm{~Hz}$. We then combined these with the parameterized response functions to predict the buoy rms tilt. Lastly, we compared the predicted buoy tilt to the rms tilt measured by the rate gyros on our ASIS, during the same time period.

Ideally, we want to compare the individual vector components of measured tilt with predicted tilt (ie. compare pitch to pitch and roll to roll). However, to do this we must know the heading of ASIS. Unfortunately, due to a compass malfunction, heading data is not available from this portion of the deployment, and so comparing the vector components of tilt is not possible. To get an upper bound on the predicted pitch of ASIS, we multiply the wave slope spectra by the magnitude of the pitch response function. Similarly, to estimate an upper bound on the predicted buoy roll, we multiply the wave slope spectra by the magnitude of the roll response function. During this period the wave direction was approximately constant from the south. We argue that because a tethered ASIS buoy makes a circle two times a day due to the tides, it therefore sees roughly equal forcing in pitch and roll. Hence the mean of our two computations should provide a reasonable estimate of tilt. Figures 4.10 and 4.11, respectively show the correlation of predicted tilt in pitch to measured tilt and the correlation of predicted tilt in roll to measured tilt. 


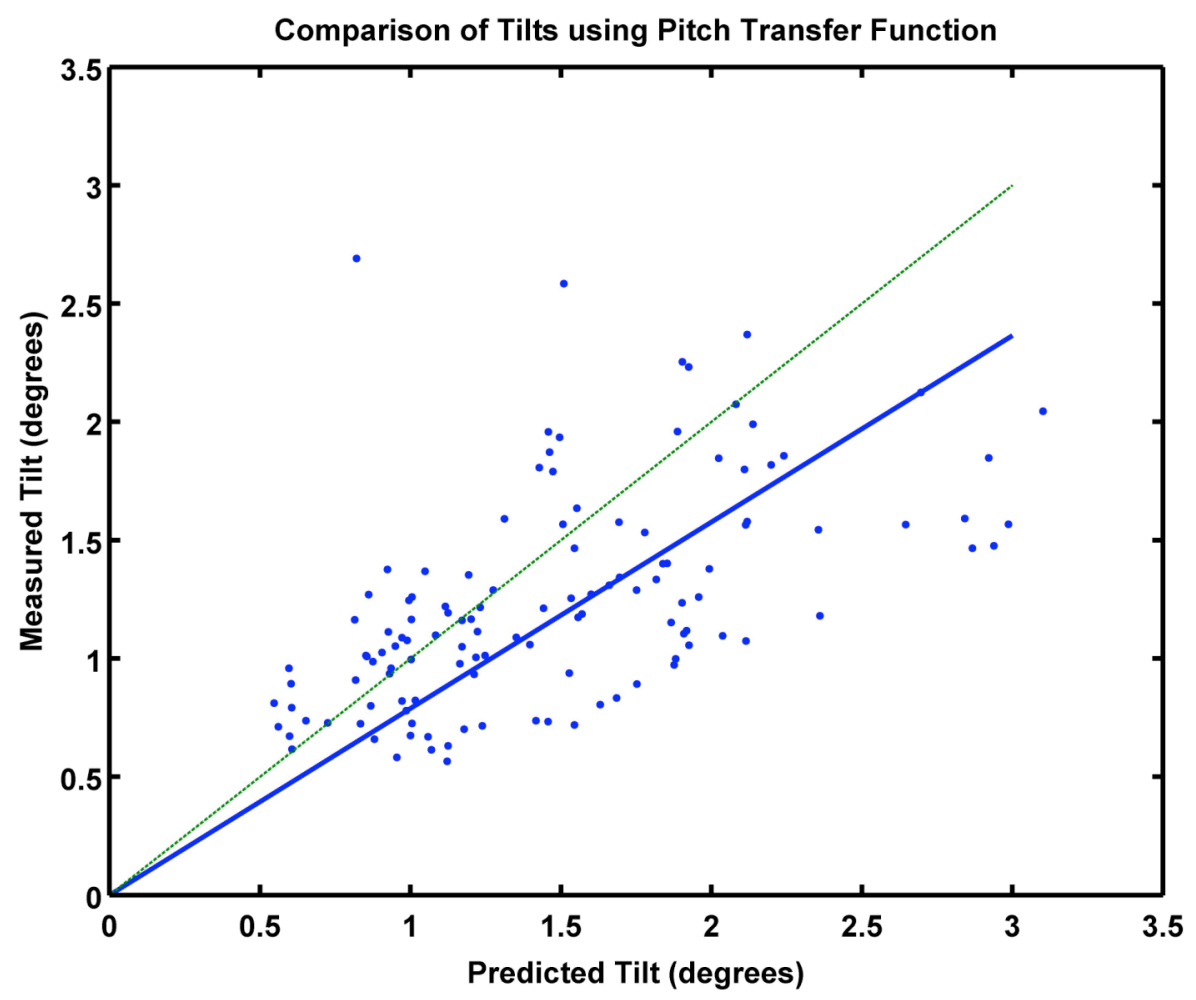

Figure 4.10 A lower bound on the correlation of predicted tilt to measured tilt. The solid line is a linear fit to the data points and has a slope of 0.79 . The dashed line is 1:1. These data have a correlation coefficient of 0.56 . 


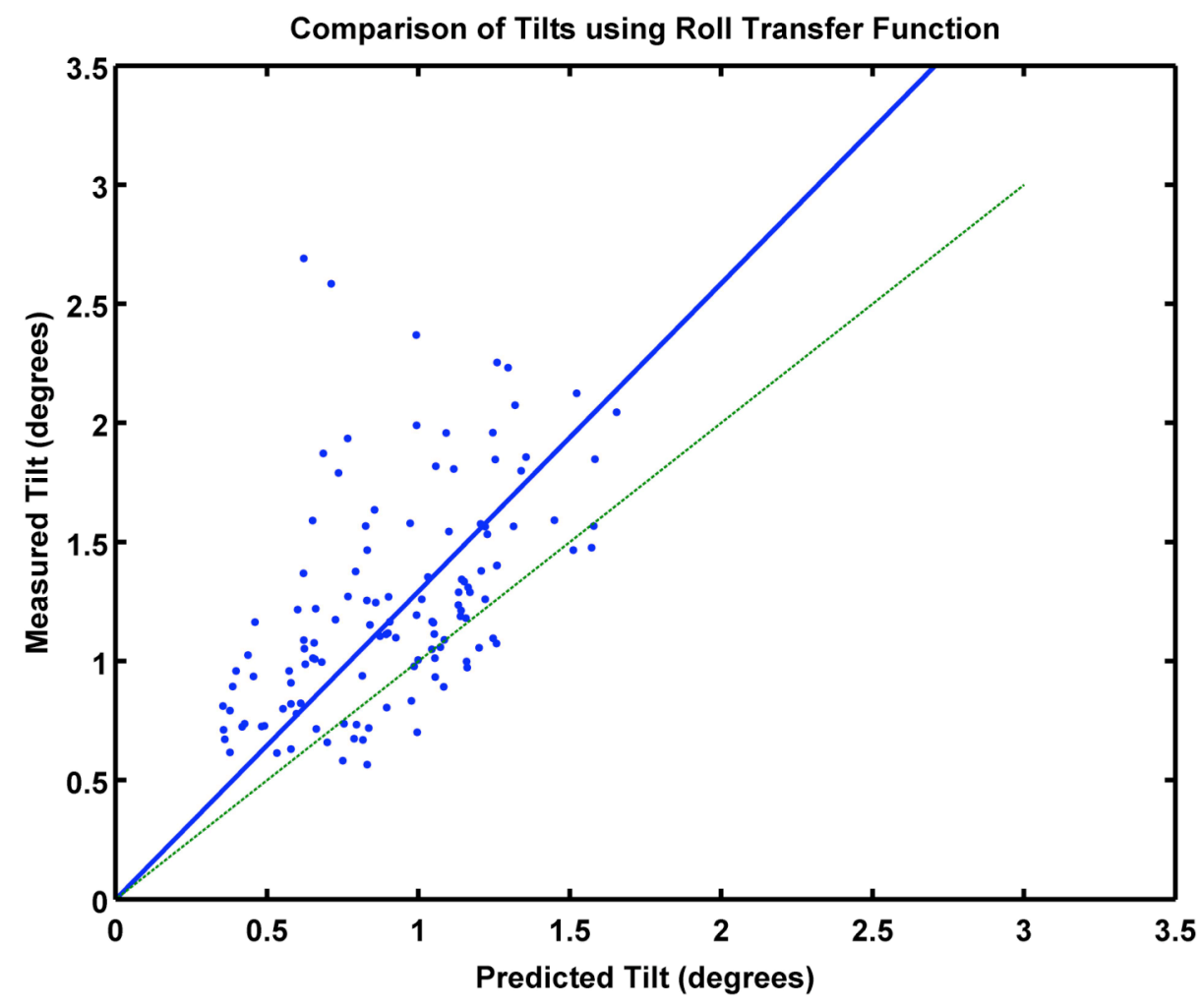

Figure 4.11 An upper bound on the correlation of predicted tilt to measured tilt. The solid line is a linear fit to the data points and has a slope of 1.3. The dashed line denotes 1:1. These data have a correlation coefficient of 0.52 .

The mean slope of the fits in Figures 4.10 and 4.11 is 1.04, suggesting that the ASIS tilt response function gives a reasonably good prediction of the buoy rms tilt.

\subsection{Modeled ASIS spectral response to waves}

In this section we use a model of the wave height spectrum due to Donelan et. al. (1985), coupled with the ASIS transfer functions given in Figure 4.9 to estimate the response of ASIS in various sea states. The latter are based on the characterization of "typical" and "high" sea states derived from NDBC buoy observations on the New England continental shelf (see Table 4.2). We have augmented these with an "extreme" condition modeled after a storm event recorded in late 2005 at the MVCO. A summary of the parameters of these three conditions is shown in Table 4.3. 


\begin{tabular}{|c|c|c|c|}
\hline Simulation parameters & "Typical" & "High" & "Extreme" \\
\hline U (m/s) & 7 & 15 & 25 \\
\hline Tp (s) & 5 & 8 & 10 \\
\hline Computed quantities & & & \\
\hline U / Cp & 0.9 & 1.2 & 1.6 \\
\hline Hs (m) & 1.2 & 3.6 & 6.3 \\
\hline
\end{tabular}

Table 4.3 Wind and wave parameters used to simulate ASIS response.

Note that we have slightly changed the parameters defining "typical" and "high" conditions for better consistency with the Donelan-Hamilton-Hui (DHH) model. We used our "typical" and "high" conditions to define parameterized fetch-limited DHH wave height spectra (Donelan et. al., 1985) which we later use for wave forcing in the model. The DHH frequency-direction spectrum

$$
F(\omega, \theta)=\frac{1}{2} \Phi(\omega) \beta \sec h^{2}\{\beta[\theta-\bar{\theta}(\omega)]\}
$$

where $\bar{\theta}$ is the mean wave direction and $\omega$ is the angular frequency $(\mathrm{rad} / \mathrm{s})$, and $\beta(\omega)$ parameterizes the directional spread and is given by

$$
\begin{array}{ll}
\beta=2.61\left(\omega / \omega_{p}\right)^{1.3} ; & 0.56<\omega / \omega_{p}<0.95 \\
\beta=2.28\left(\omega / \omega_{p}\right)^{-1.3} ; & 0.95<\omega / \omega_{p}<1.6 \\
\beta=1.24 ; & \text { otherwise. }
\end{array}
$$

The non-directional frequency spectrum is

$$
\begin{array}{ll}
\Phi(\omega)=\alpha g^{2} \omega^{-5}\left(\omega / \omega_{p}\right) \exp \left\{-\left(\frac{\omega_{p}}{\omega}\right)^{4}\right\} \gamma^{\Gamma} \\
\alpha=0.006\left(U_{c} / c_{p}\right)^{0.55} ; \quad 0.83<U_{c} / c_{p}<5 . \\
\gamma=1.7 ; & 0.83<U_{c} / c_{p}<1 ; \\
\gamma=1.7+6.0 \lg \left(U_{c} / c_{p}\right) ; & 1 \leq U_{c} / c_{p}<5
\end{array}
$$


where $\Gamma=\exp \left\{-\left(\omega-\omega_{p}\right)^{2} / 2 \sigma^{2} \omega_{p}^{2}\right\}$,

$$
\sigma=0.08\left[1+4 /\left(U_{c} / c_{p}\right)^{3}\right] ; \quad 0.83<U_{c} / c_{p}<5
$$

and $U_{c}$ is the average speed of $10 \mathrm{~m}$ wind in the direction of the waves at the peak of the spectrum, and $c_{p}$ is the phase speed at the peak frequency. The parameter $U_{c} / c_{p}$, known as the "inverse wave age", is a measure of wave development. The non-directional DHH spectra used to simulate our three classes of wind and wave conditions are shown in Figure 4.12.
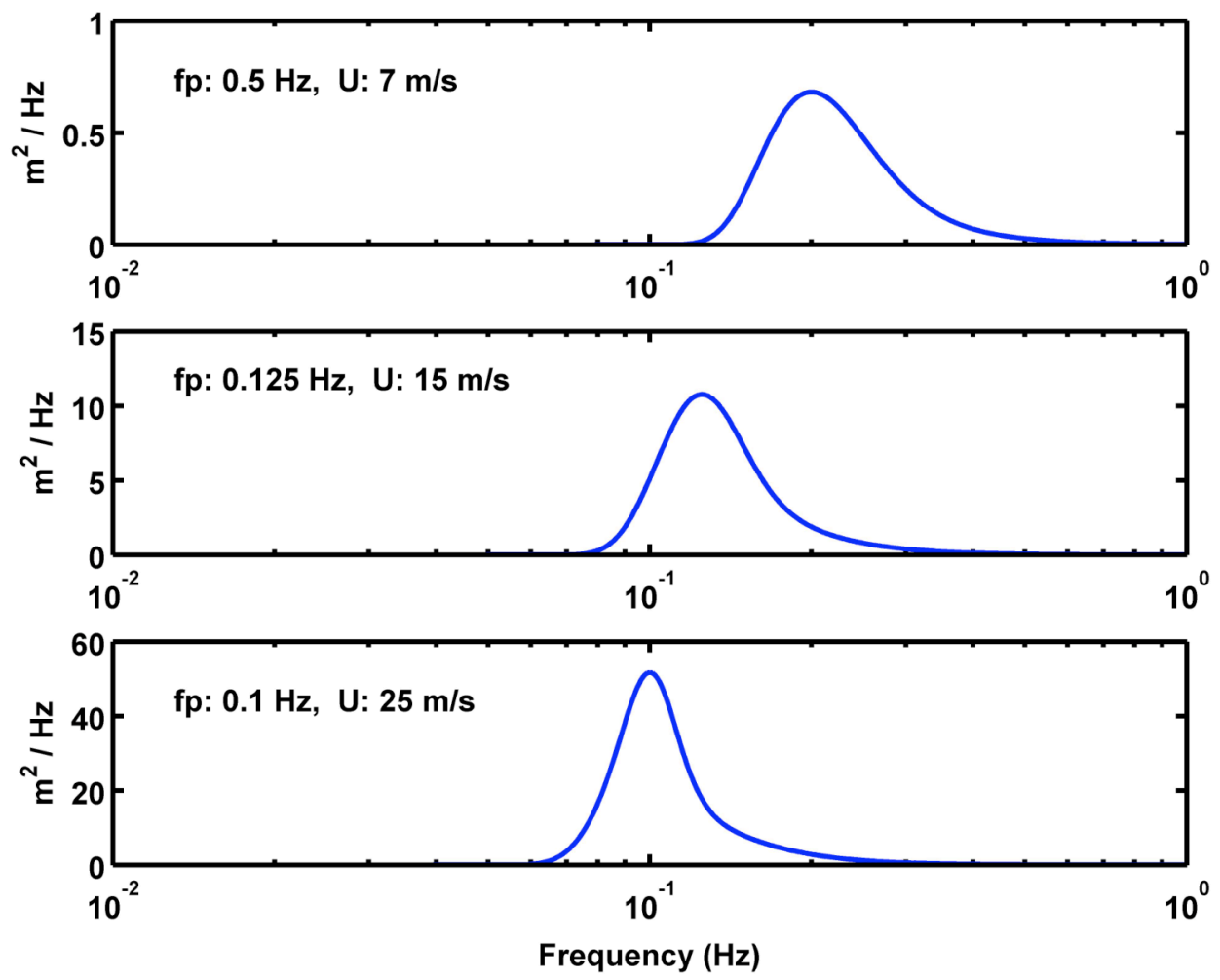

Figure 4.12 Wave forcing as defined by DHH spectra for "typical", "high" and "extreme" cases.

The spectral response of ASIS to the wave forcing defined by the DHH spectra is shown in Figure 4.13. The rms responses of ASIS are listed in Table 4.4. Here, the significant 
response amplitude is defined as the average of the response to the one-third highest waves.
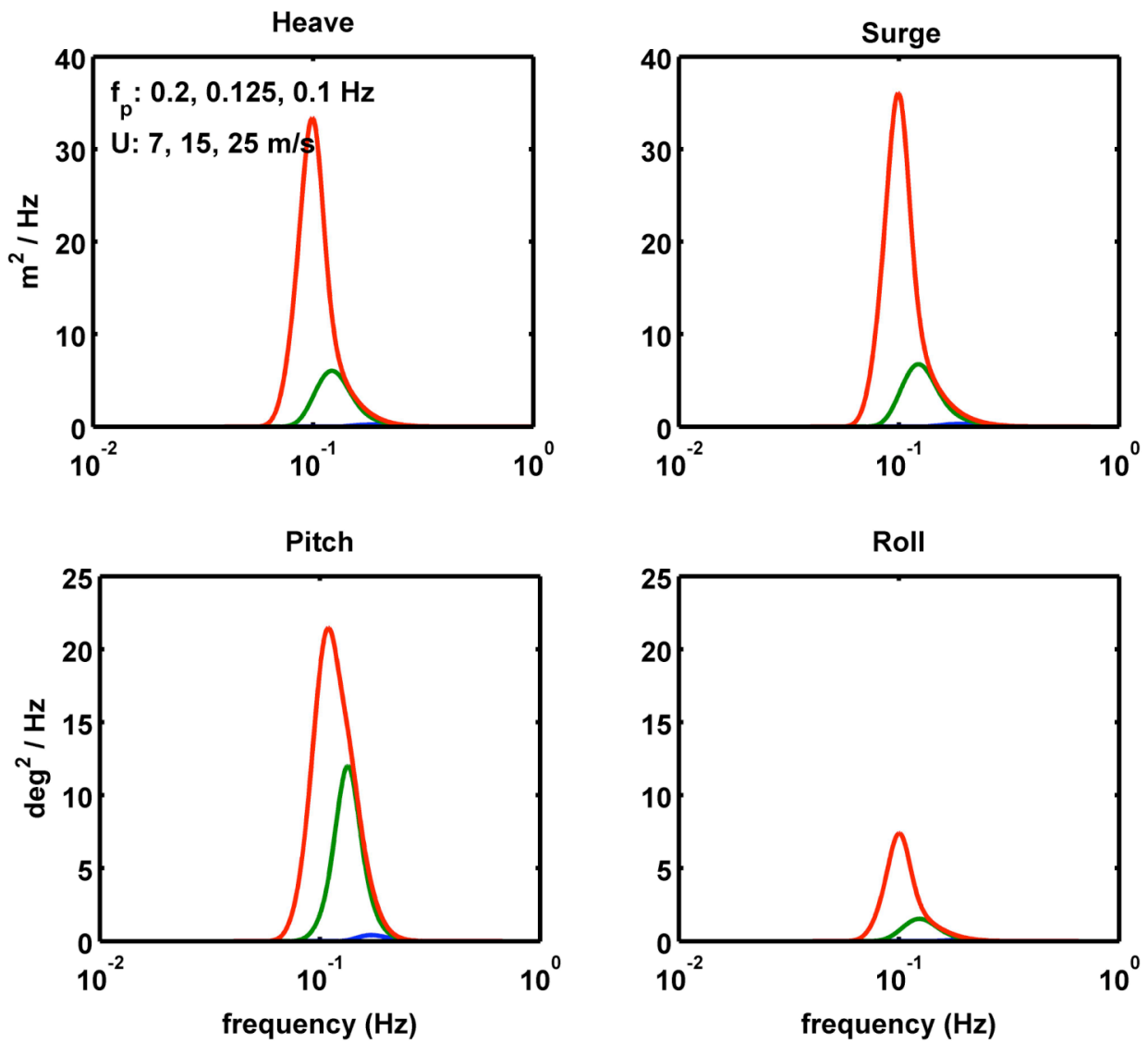

Figure 4.13 ASIS spectral responses due to DHH wave forcing for the three cases (blue has values of $f_{P}=0.2 \mathrm{~Hz}, \mathbf{U}=7 \mathrm{~m} / \mathrm{s}$; green has values of $f_{P}=0.125 \mathrm{~Hz}, \mathbf{U}=15$ $\mathrm{m} / \mathrm{s}$; and red has values of $f_{P}=0.1 \mathrm{~Hz}, \mathrm{U}=25 \mathrm{~m} / \mathrm{s}$ ).

\begin{tabular}{|c|c|c|c|}
\hline Significant Response Amplitude & "Typical" & 'High" & "Extreme" \\
\hline Wave height (m) & 1.2 & 3.6 & 6.3 \\
\hline ASIS Heave (m) & 0.59 & 2.5 & 4.7 \\
\hline ASIS Pitch (deg) & 0.65 & 3.1 & 4.6 \\
\hline ASIS Roll (deg) & 0.30 & 1.3 & 2.3 \\
\hline ASIS Surge (m) & 0.72 & 2.7 & 5.0 \\
\hline
\end{tabular}

Table 4.4 ASIS rms responses to wave forcing for "typical", "high" and "extreme" cases. 


\subsection{Modeled ASIS temporal response to waves}

It is evident from Figure 4.13 and Table 4.4 that ASIS responds more to the low frequency, energetic, waves commonly seen in high sea states. However, to understand how well ASIS follows the surface it is necessary to study the time-history of the buoy response. To do this, we use the DHH spectra defined in Figure 4.12 to simulate a random time series with Gaussian statistics. This should produce a good estimate of the sea surface for the "typical" and "high" sea states. In the "extreme" case, non-linearities (bound harmonics) can increase in skewness of the sea surface. Although in deep water the non-linearities do not affect the wave velocity, the waves we synthesize in the "extreme" case may slightly under-estimate the forcing in heave.

Figure 4.14 plots the time series of wave height in blue and ASIS heave response in red for the three defined classes of sea state. As expected, ASIS does not follow the shorter waves characteristic of "typical" conditions as well as it follows the longer period waves that dominate the "high" and "extreme" sea states. 
fp: $0.5 \mathrm{~Hz}, \mathrm{U}: 7 \mathrm{~m} / \mathrm{s}$; rel error: 0.61

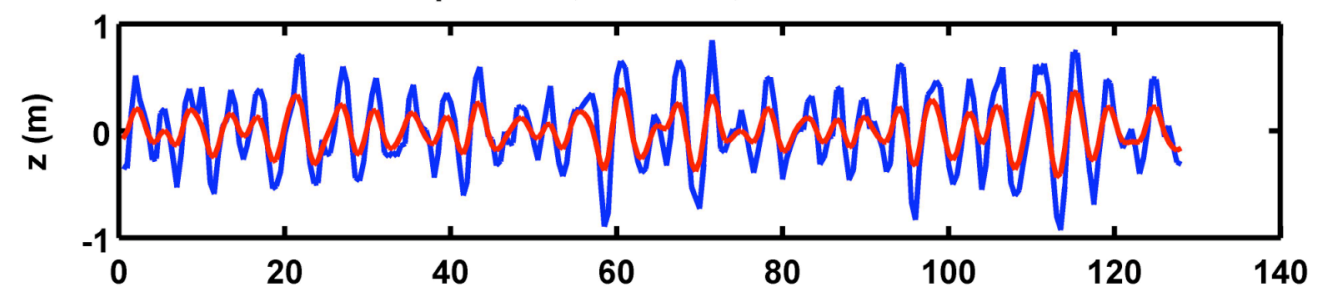

fp: $0.125 \mathrm{~Hz}, \mathrm{U}: 15 \mathrm{~m} / \mathrm{s}$; rel error: 0.45

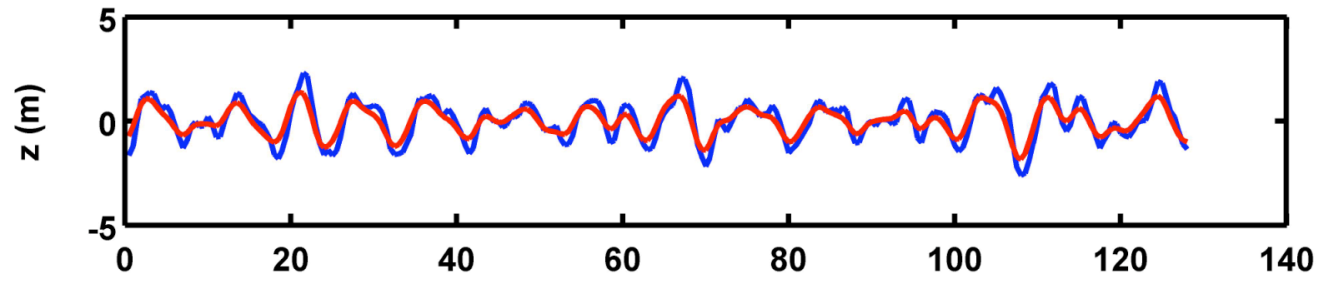

fp: $0.1 \mathrm{~Hz}, \mathrm{U}: 25 \mathrm{~m} / \mathrm{s}$; rel error: 0.36

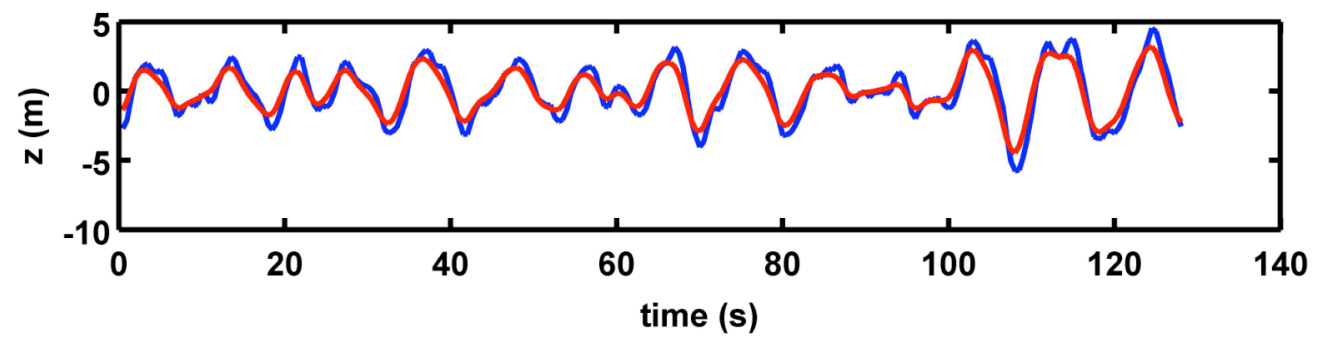

Figure 4.14 ASIS response to time series of sea surface height for the three cases.

\subsection{Wave overtopping probabilities}

Our simulated time series can also be used to study wave overtopping. We simulate about 35.6 hours of wave forcing at a $2 \mathrm{~Hz}$ sample rate for the cases defined in Table 4.3. In the conceptual ASIS design discussed in section 3.3, the sodar antenna box is located $2.3 \mathrm{~m}$ above the mean waterline (MWL) of ASIS. We consider a wave exceeding this height to be overtopping. Our simulation produced no overtopping in the "typical" and "high" classes. However, in the "extreme" class, we predicted that eight out of about 12,800 simulated waves would overtop the sodar antenna box. Seven out of the eight waves were overtopping for a period of 0.5 seconds and one was overtopping for a period of 1 second. A plot of overtopping waves for our simulated time series is shown in Figure 4.15. The lower panel shows surface displacement relative to the mean sea surface, with overtopping waves denoted as red stars. The upper panel shows surface displacement relative to the MWL of ASIS. Again, overtopping waves are denoted with 
red stars. A dotted line marks the height of the sodar above MWL (2.3 m) in both panels. It is noticeable that overtopping waves were not necessarily the highest waves in the time series. This shows that the phase of the ASIS response plays a central role in determining the percentage of waves that will overtop.
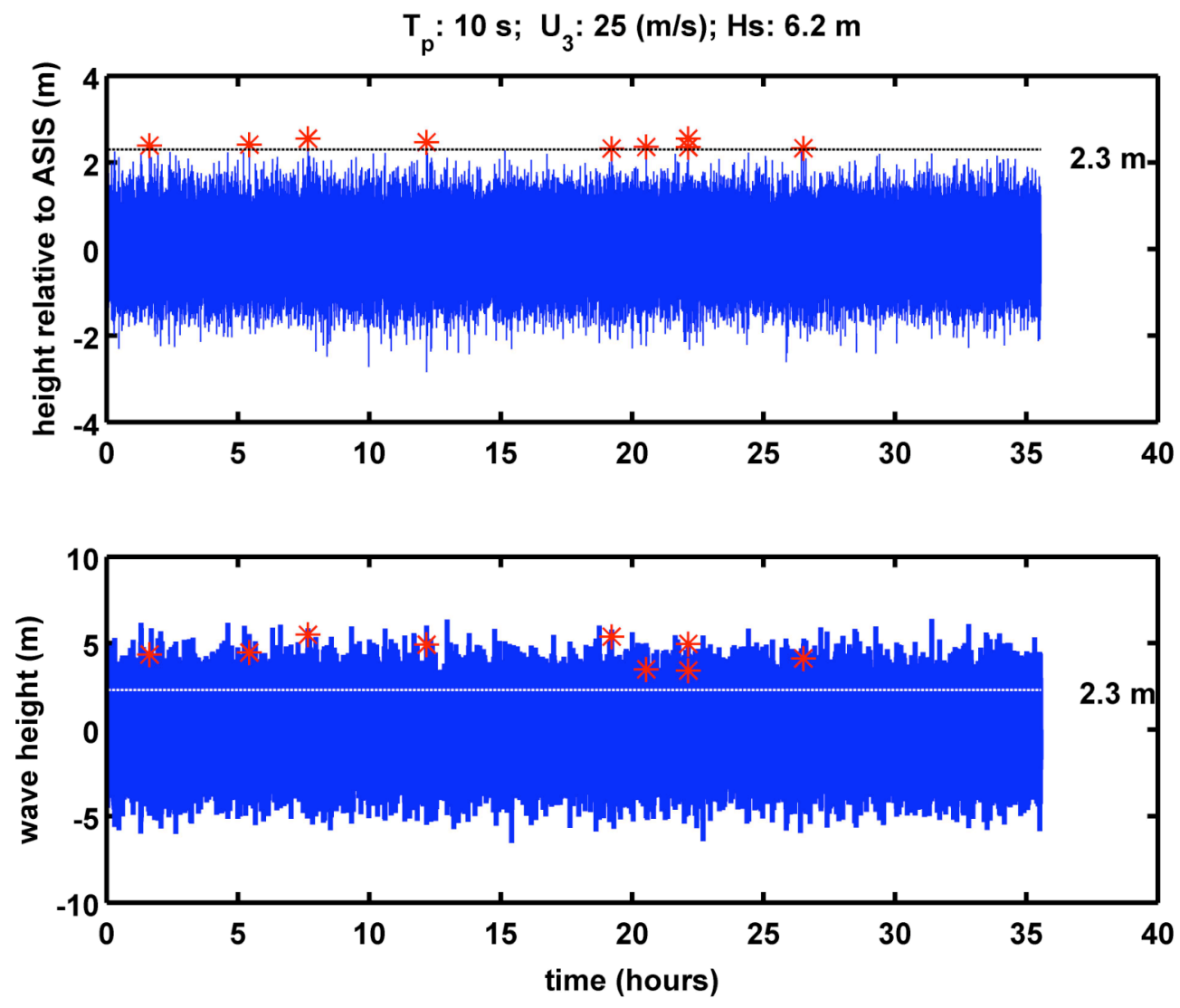

Figure 4.15 The height of waves relative to MWL of ASIS (top panel) and the corresponding height of waves relative to the mean sea surface (bottom panel). The height of the sodar above MWL $(2.3 \mathrm{~m})$ is drawn in with a dotted line in both panels.

\subsection{ASIS tilt response at sub-waveband frequencies}

In this section, we compute ASIS tilts at frequencies below the wave band to get an understanding of the buoy's low frequency response. At low frequencies, lateral accelerations are negligible, so the accelerometer output at these frequencies measures the actual tilt. 
A model was developed in order to predict ASIS buoy tilt at low frequency in response to the drag induced by winds and currents (see Appendix C). In order to validate it, we compared the predicted tilt to 20 -minute averages of buoy tilt as measured by the accelerometer and compass tilt sensor during our deployment (Figure 4.16). Tilts are generally seen to be small, less than $1.5^{\circ}$, and agreement between the model prediction and the accelerometer-based estimate of tilt is within $0.5^{\circ}$. Differences between the model and measured tilts may be due to the fact that the wind and current data used to force the buoy in the model were taken from the MVCO 12-m node and the shore tower, whereas the ASIS buoy was deployed $\sim 3 \mathrm{~km}$ further offshore $(0.5 \mathrm{~km}$ south of the MVCO tower).

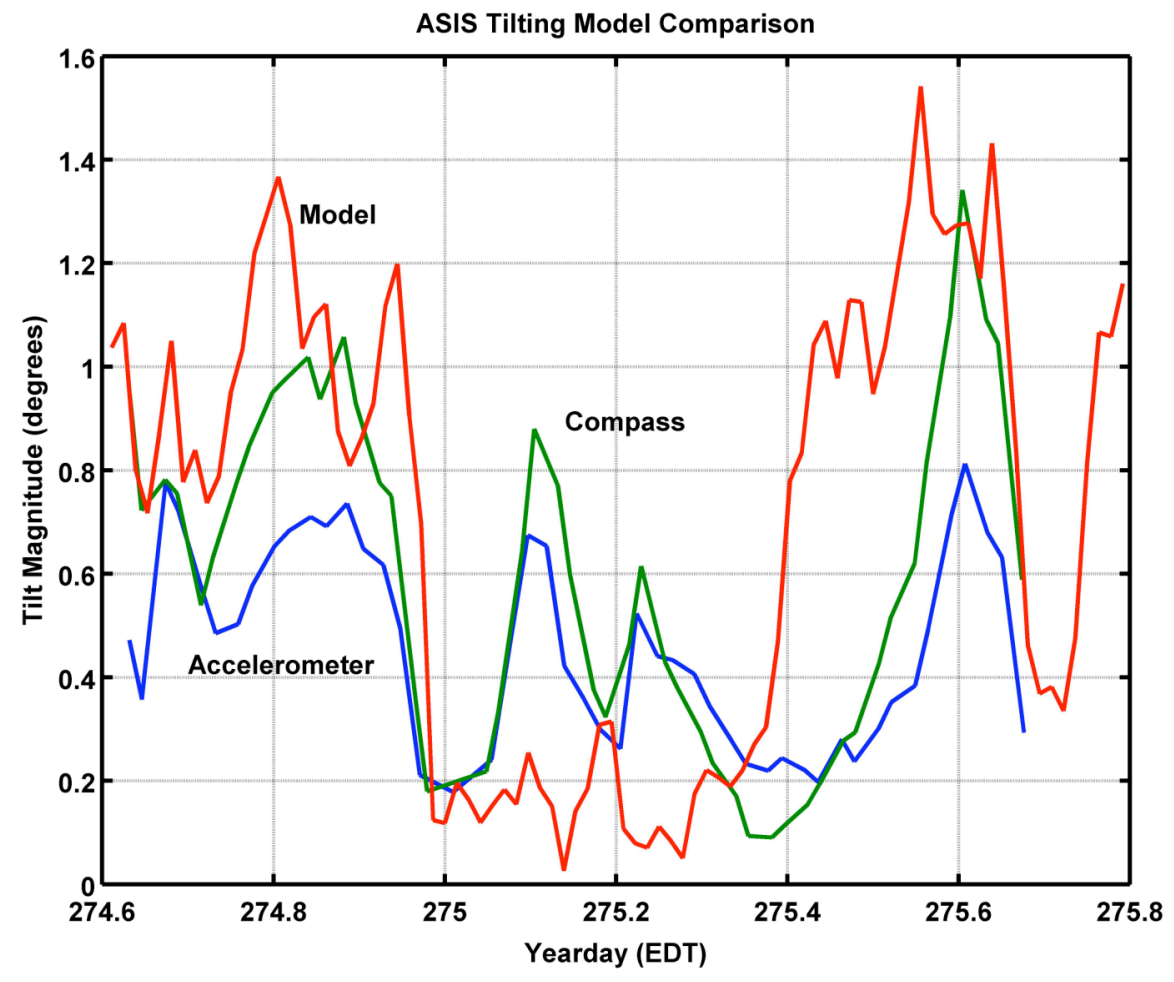

Figure 4.16 ASIS sub-waveband tilt model compared with data from the accelerometers and the compass tilt sensor. 


\subsection{Wave band tilts}

In this section we use data to examine ASIS tilts in the wave band for different sea states. It is important to understand the distributions of measured ASIS tilts in response to waves. The tilts in the wave band are computed by high-pass filtering and integrating the rate gyro data. In this section, we compute heave, pitch and roll for three sea states characterized by the amount of $r m s$ angular rate: low $r m s \Omega\left(1.05^{\circ} / \mathrm{s}\right)$, mid $r m s \Omega$ $(1.72 \% \mathrm{~s})$, and high $r m s \Omega\left(2.35^{\circ} / \mathrm{s}\right)$. The data used in this section are taken from our ASIS deployment (described in Appendix B).

Figures 4.17, 4.18, and 4.19 show histograms of measured ASIS tilts in pitch, roll and yaw for the three sea states defined above. As expected, the distributions of tilt are approximately Gaussian, with the majority of tilts being under $2^{\circ}$. A summary of the standard deviations of tilt for the three sea states is given in Table 4.5

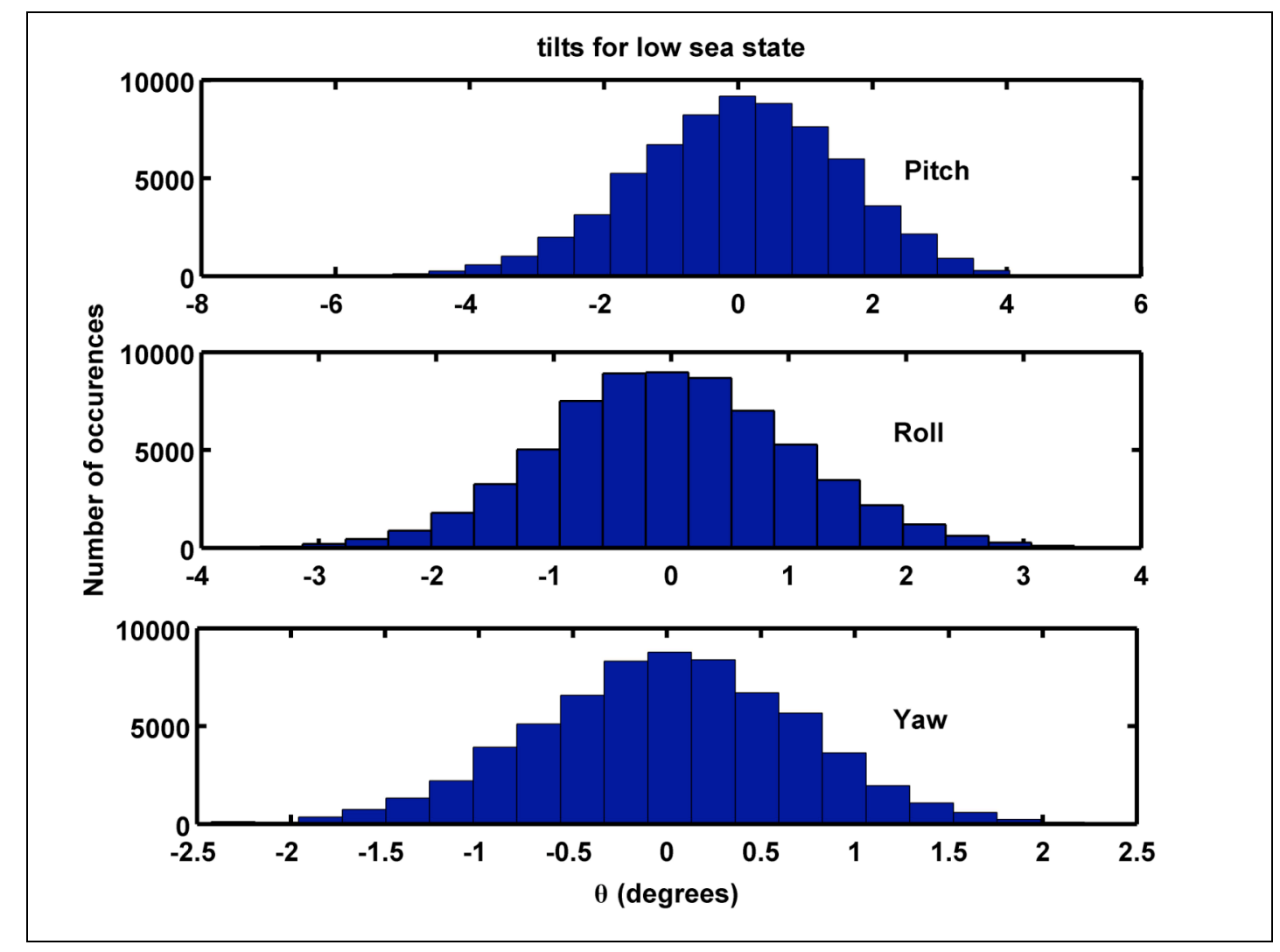

Figure 4.17 Histograms of pitch, roll and yaw in the waveband for the low sea state case. 


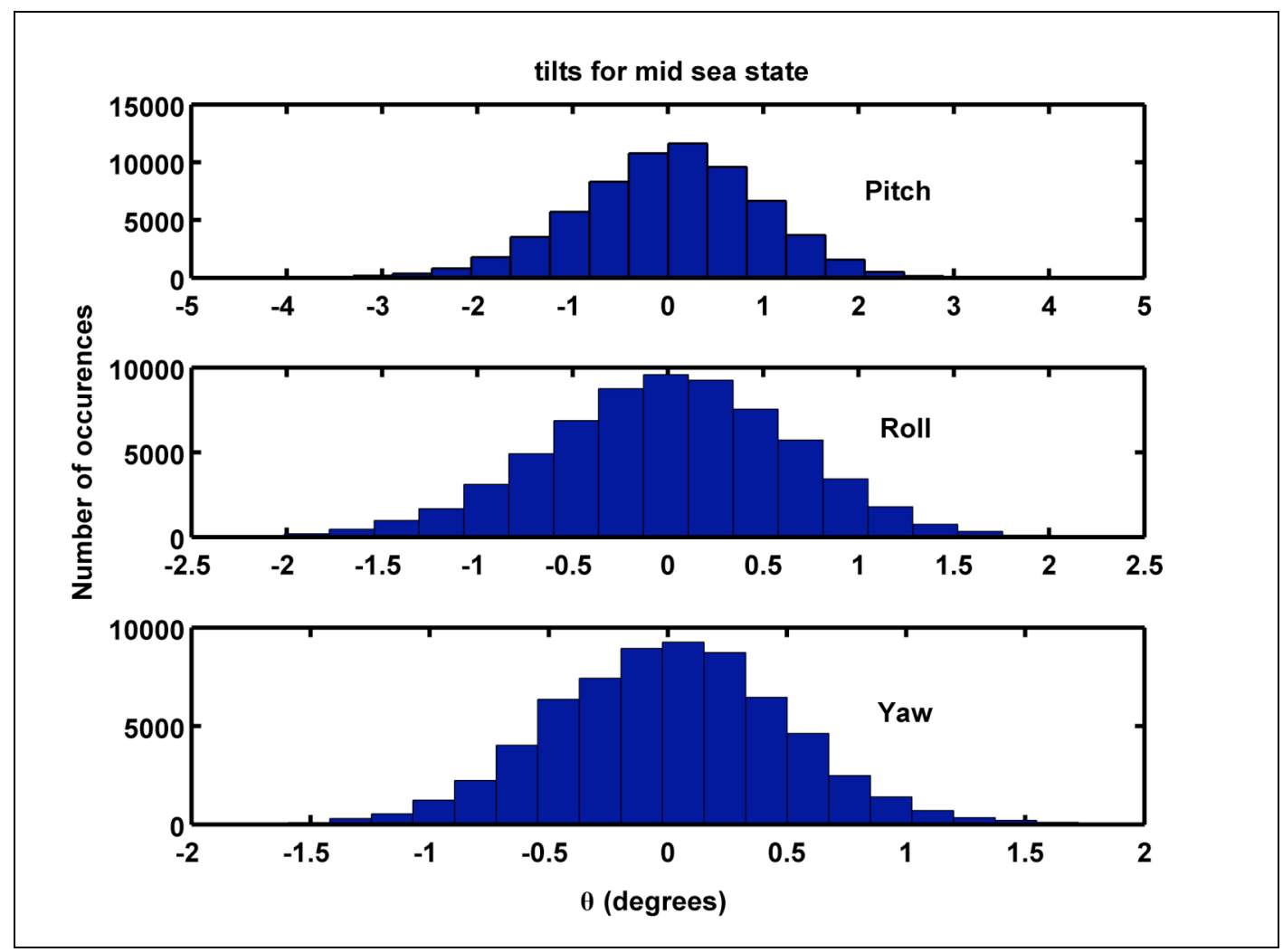

Figure 4.18 Histograms of pitch, roll and yaw in the waveband for the mid sea state case. 


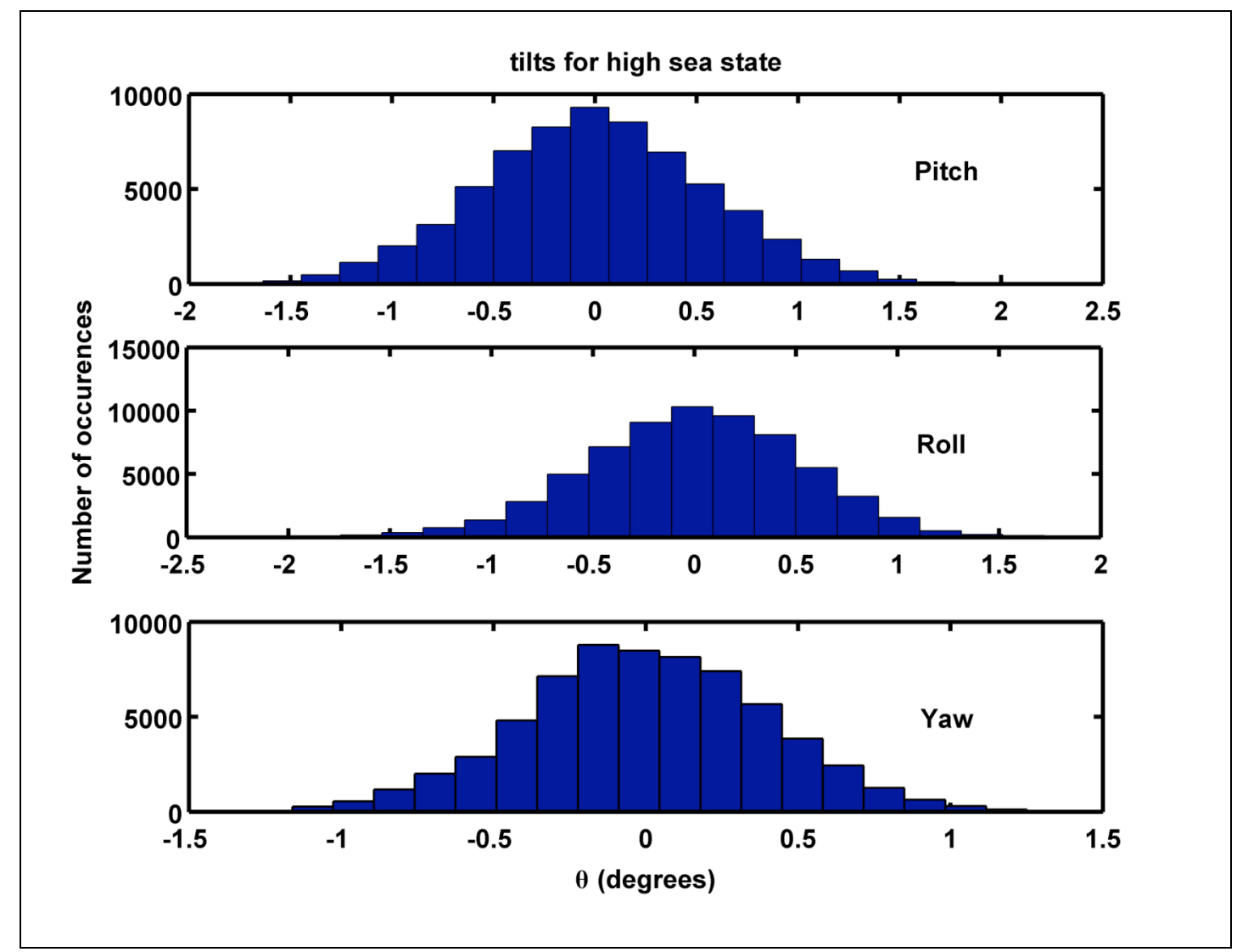

Figure 4.19 Histograms of pitch, roll, and yaw in the waveband for the high sea state case.

\begin{tabular}{|c|c|c|c|}
\hline \multicolumn{5}{|c|}{ Standard deviations of tilt (degrees) } \\
\hline Tilt direction & Low sea state & Mid sea state & High sea state \\
\hline Pitch & 0.56 & 0.97 & 1.53 \\
\hline Roll & 0.53 & 0.64 & 1.05 \\
\hline Yaw & 0.40 & 0.49 & 0.70 \\
\hline
\end{tabular}

Table 4.5 Summary of standard deviations of tilt for the three sea states defined in section 4.9.

\subsection{ASIS drift response to combined wind and wave forcing}

In this section, we consider the response of the ASIS "system" (consisting of buoy, tether, and surface mooring) to combined wind and wave forcing. One tool with which to accomplish this is the ASIS drift model (described in Appendix E). Unfortunately, our deployed ASIS only carried an ARGOS position locater that could not resolve the motion 
of the buoy over its $50 \mathrm{~m}$ radius watch circle. However, this model can be used to predict the heading of tethered and un-tethered ASIS buoys for given winds and currents. The model also predicts when the tether becomes slack. It is important to know what conditions will cause this to happen because a slack tether can lead to chaffing and a premature separation of the buoy from its mooring (this will be discussed in more detail later in this section).

Although we only obtained about 28 hours of heading data from our deployment of ASIS, due to the malfunction of our PNI compass, we can compare these data to our model as a check on the model accuracy. The wind and current velocities used in the model were taken from the MVCO (12-m node and shore tower). The measured ASIS heading compared to the ASIS prediction for 01-02 October 2005 is shown in Figure 4.20. The data is averaged to one minute and heading is in degrees true in the direction the tether is streaming from. The vectoral components of the wind and currents as well as the tension in the tether for this time period are shown in Figures 4.21 and 4.22, respectively. In this case, the maximum tension in the tether was approximately $67 \mathrm{lbs}$. It is evident that the model shows agreement with the data during periods when the tension in the tether is relatively large, and when the direction of winds and currents align. The agreement is worse during periods when winds and currents are misaligned. This may be due to rotation of the buoy about the tether attachment point caused by the misalignment of winds and currents.

One way to improve the model in the future would be to equip ASIS with an anemometer, a downward-looking ADCP, and a GPS. These would provide measurements of winds, currents, buoy velocity over the bottom and buoy location, thus improving knowledge of the wind and current forcing as well as providing higher resolution measurements of buoy position and motion. 


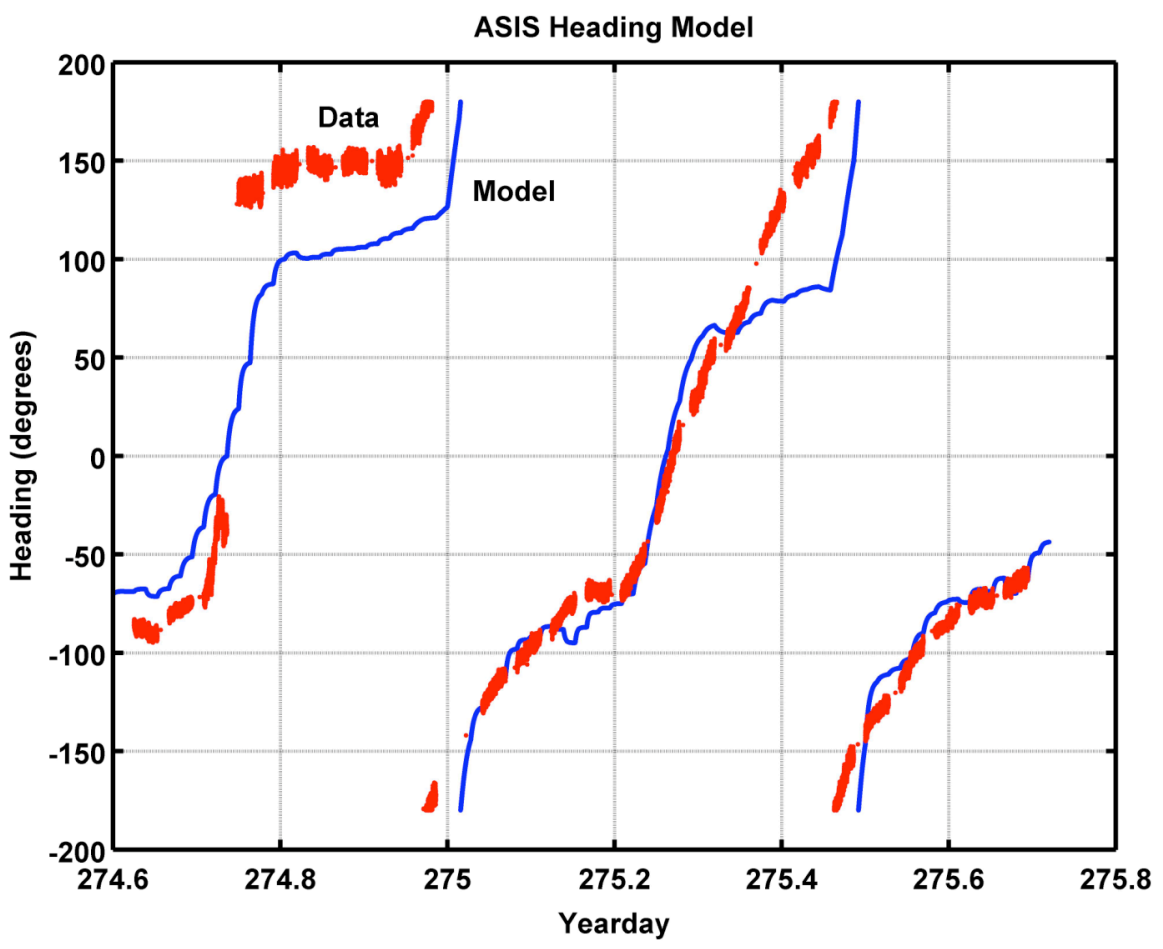

Figure 4.20 ASIS Drift Model comparison to data for 01-02 October 2005 (YD 274275). 

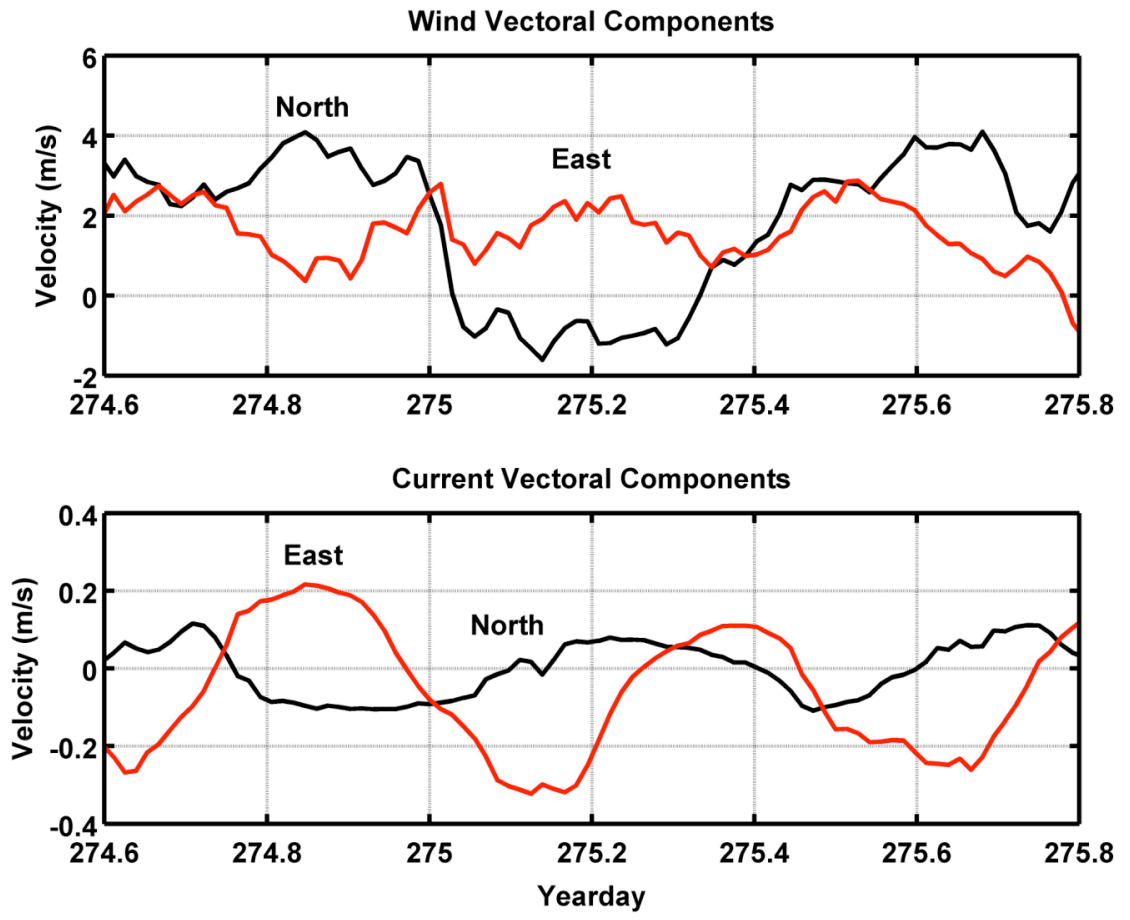

Figure 4.21 Wind and current vectors.

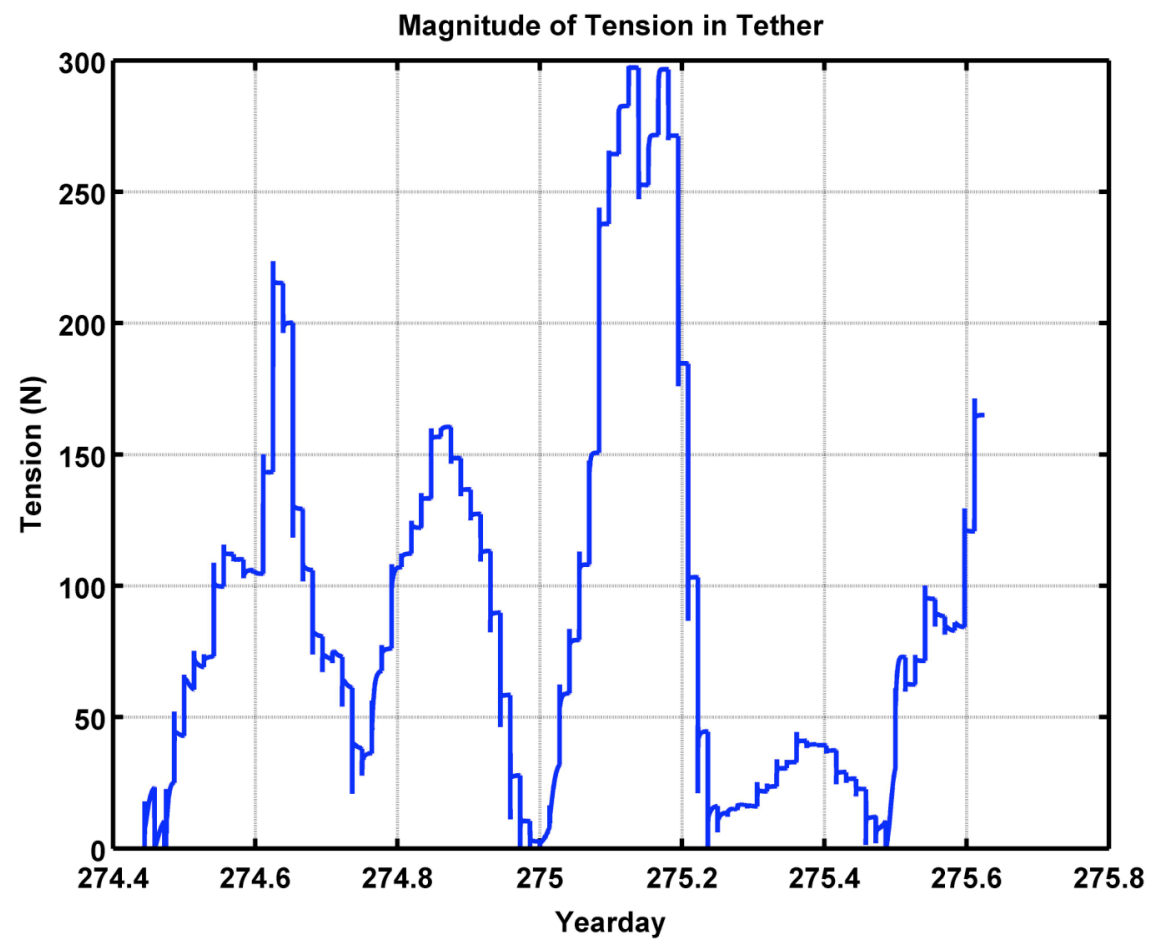

Figure 4.22 Magnitude of tension in the tether. 
Since the ASIS drift model shows reasonably good agreement with the heading data, we use it further to predict the ASIS response to combined wind and wave forcing. We are interested answering two questions: [1] How fast does ASIS reach an equilibrium speed after a change in forcing? and [2] Under what conditions does ASIS's tether become slack?

\section{[1] ASIS response to changes in current}

Our drift model is time-dependent, so we can use it to compute the time it takes ASIS to respond to changes in the current. It takes a free-drifting ASIS 62.5 seconds to reach a velocity that is $97 \%$ of a current velocity of $0.5 \mathrm{~m} / \mathrm{s}$ (current velocity is changed instantaneously at $\mathrm{t}=0$ seconds from $0 \mathrm{~m} / \mathrm{s}$ to $0.5 \mathrm{~m} / \mathrm{s}$ ). A plot of ASIS's velocity in time is given in Figure 4.23. A non-dimensional time scale, $\tau$, for changes in velocity can be defined as

$$
\tau=\frac{(\alpha U)}{M_{v}} t
$$

where $t$ is time (s), $\alpha$ is the effective drag coefficient $\left(\frac{N}{(m / s)^{2}}\right), \mathrm{U}$ is current velocity $(\mathrm{m} / \mathrm{s})$, and $M_{v}$ is buoy virtual mass $(\mathrm{kg})$. A plot of $\left(V_{f}-V\right) / V_{f}$, versus $\tau$, where $\mathrm{V}$ and $V_{f}$ are the buoy's instantaneous and final velocity, is shown in Figure 4.24 for $\frac{\alpha}{M_{v}}=1$ and $U=0.1,0.5$ and $1 \mathrm{~m} / \mathrm{s}$ ). It is evident that ASIS accelerates rapidly at first before relaxing to its final velocity. The figure shows that ASIS's response to currents collapses under this scaling. Since the buoy's response time is small compared to a tidal cycle, a quasi-static model is appropriate for predicting response to changes in current.

It is important to note that this scaling is expected to break down when the wind forcing becomes comparable to that due to currents. Such dynamical environments are discussed in the next section. 


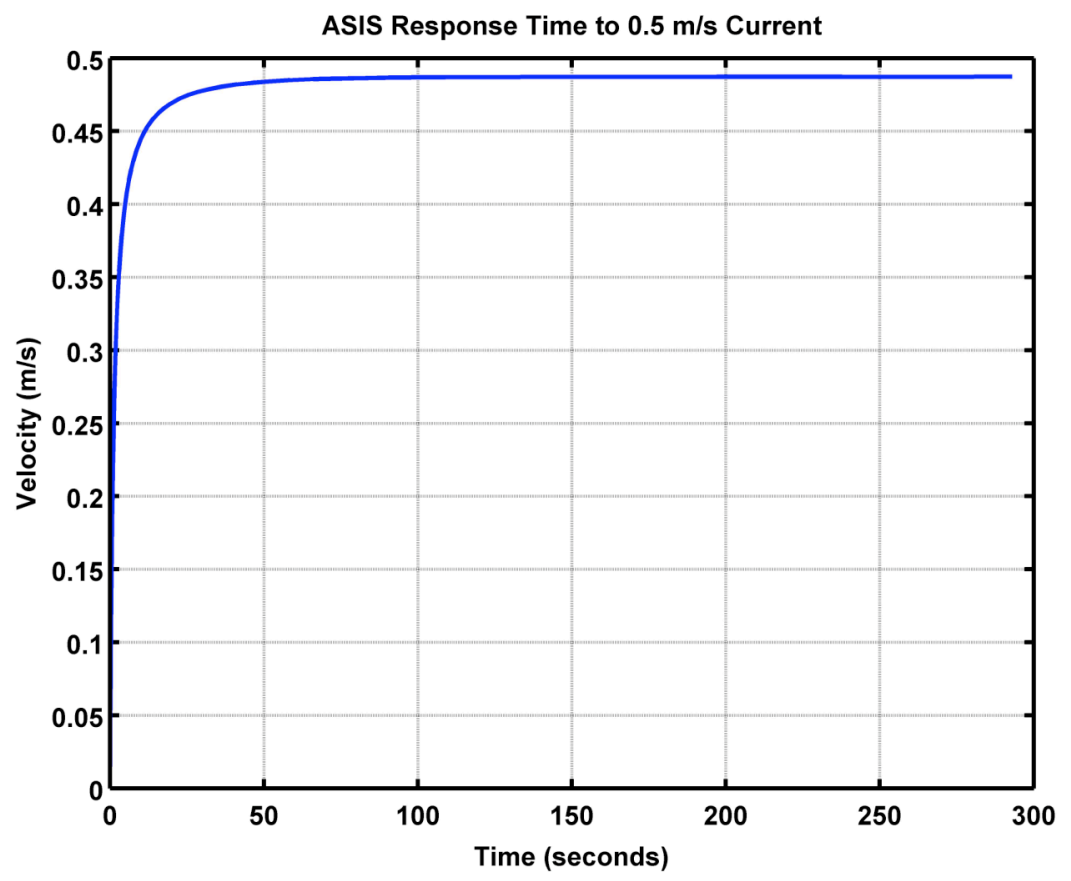

Figure 4.23 ASIS response to a $0.5 \mathrm{~m} / \mathrm{s}$ current and no winds.

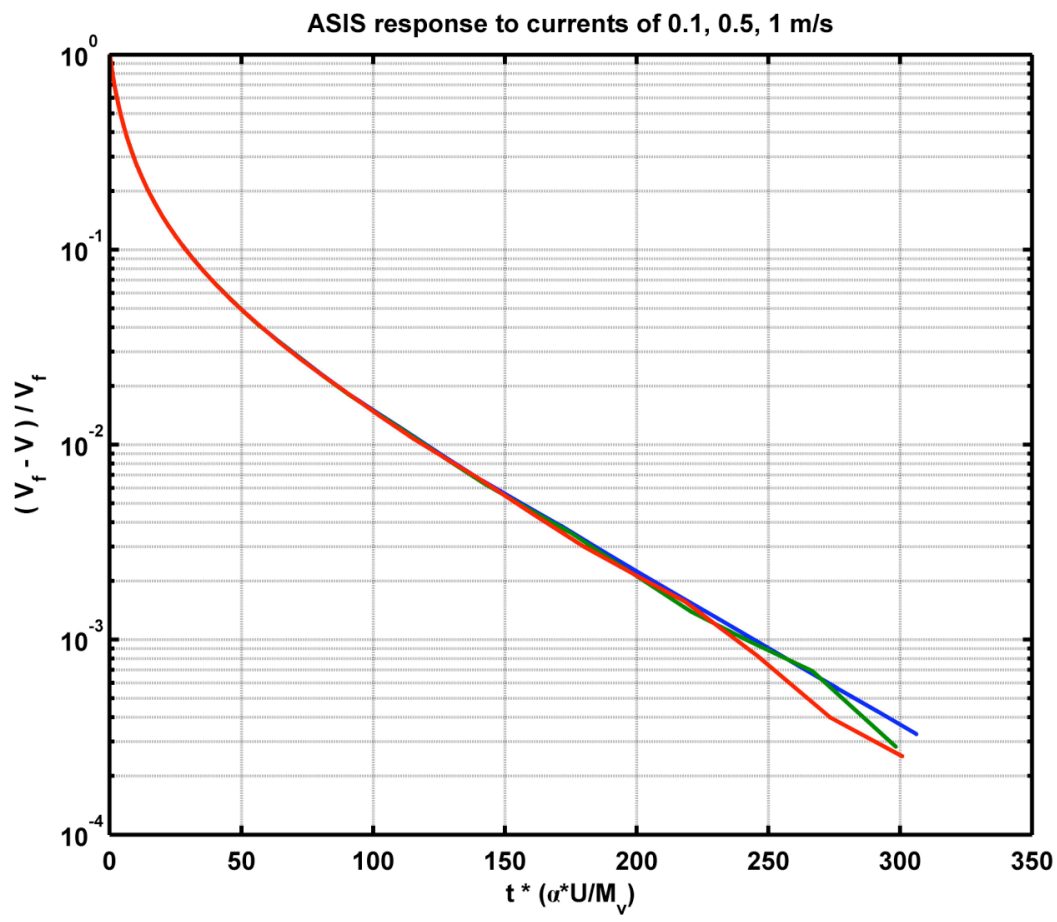

Figure 4.24 ASIS response to currents of $0.1,0.5$ and $1 \mathrm{~m} / \mathrm{s}$ for no winds. 


\section{[2] ASIS tether tension}

The tethered ASIS buoy can experience fouling of its mooring line when the tether tension changes from taut to slack and vice versa. This is a potential problem because fouling and chaffing can cause ASIS to go adrift. Therefore, it is useful to compute tether tension over typical a given tidal cycle for different wind conditions. We base our simulation on currents measured at the MVCO over a tidal cycle on 1 January 2005. We take the wind direction to be from the south, which is typical for the New England shelf, and consider wind speeds of $1,5,10$ and $15 \mathrm{~m} / \mathrm{s}$. The phase and amplitude of the measured tidal velocity (20 minute averages) for 1 January 2005 are shown in Figure 4.25. Phases are measured relative to the wind vector using the usual mathematical convention (i.e. positive counterclockwise). The tidal velocity rotates clockwise with a peak velocity of $0.25-0.3 \mathrm{~m} / \mathrm{s}$ and an average magnitude of $0.14 \mathrm{~m} / \mathrm{s}$.
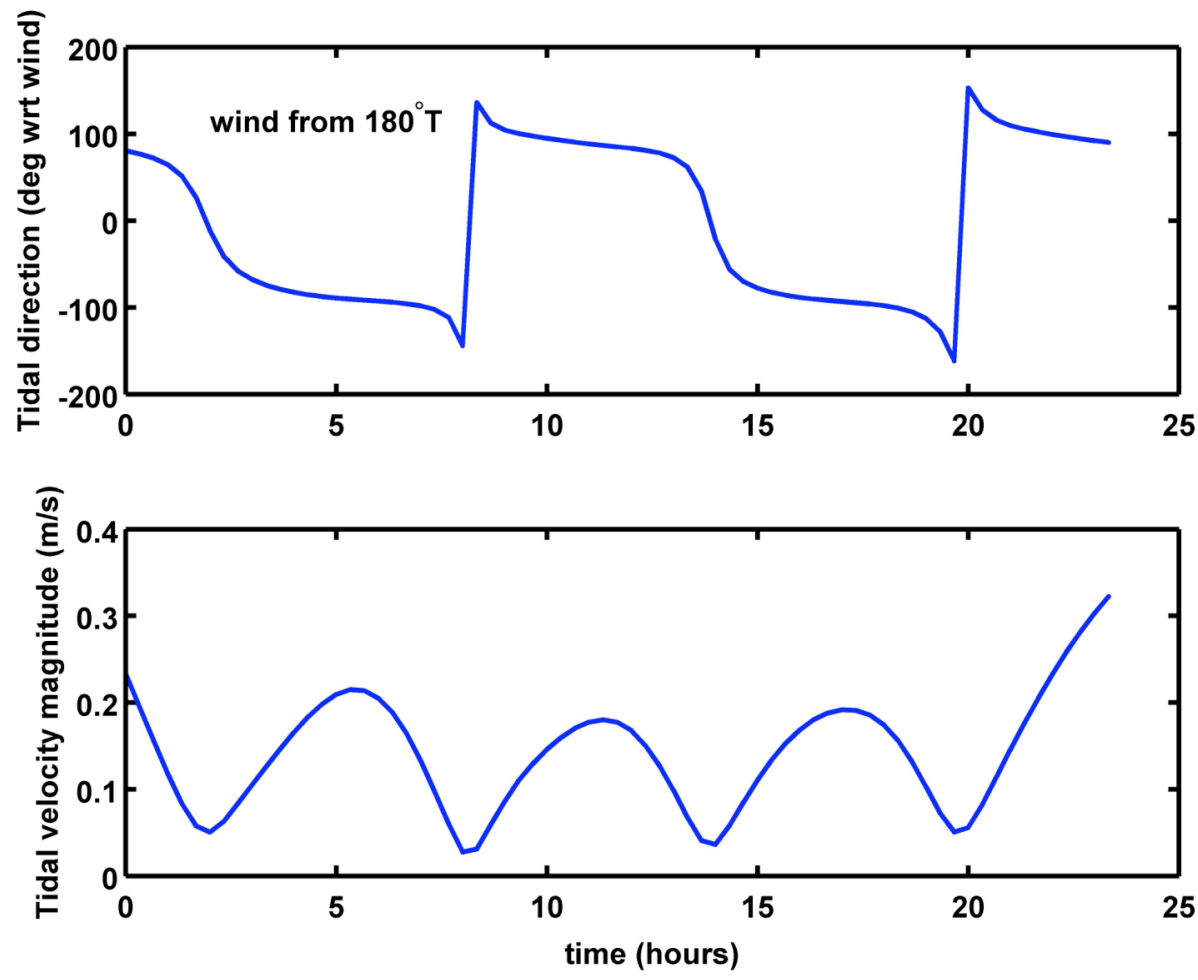

Figure 4.25 Amplitude and phase of MVCO tidal current for 1 January 2005. Phase is referenced to the direction of the wind vector (north, in this case).

The steady state response of ASIS to the tide shown in the figure above and assumed southerly winds of $1,5,10$, and $15 \mathrm{~m} / \mathrm{s}$ is shown in Figure 4.26 . At a wind speed of $1 \mathrm{~m} / \mathrm{s}$ 
the direction of the tether mimics the tidal velocity. As wind speed increases, the degree to which the tether follows decreases. At a wind speed of $15 \mathrm{~m} / \mathrm{s}$, the tether moves little in response to the tidal velocity. At this wind speed the tension in the tether is within $2 \%$ of the drag due to the wind alone. At winds speeds below $5 \mathrm{~m} / \mathrm{s}$ the drag due to current contributes $50-100 \%$ of the tension. However, tension is never that large. Even in the high wind speed case, the mean tension in the tether is roughly $100 \mathrm{lbs}$. A summary of the tether tension for different wind speeds is given in Table 4.6.
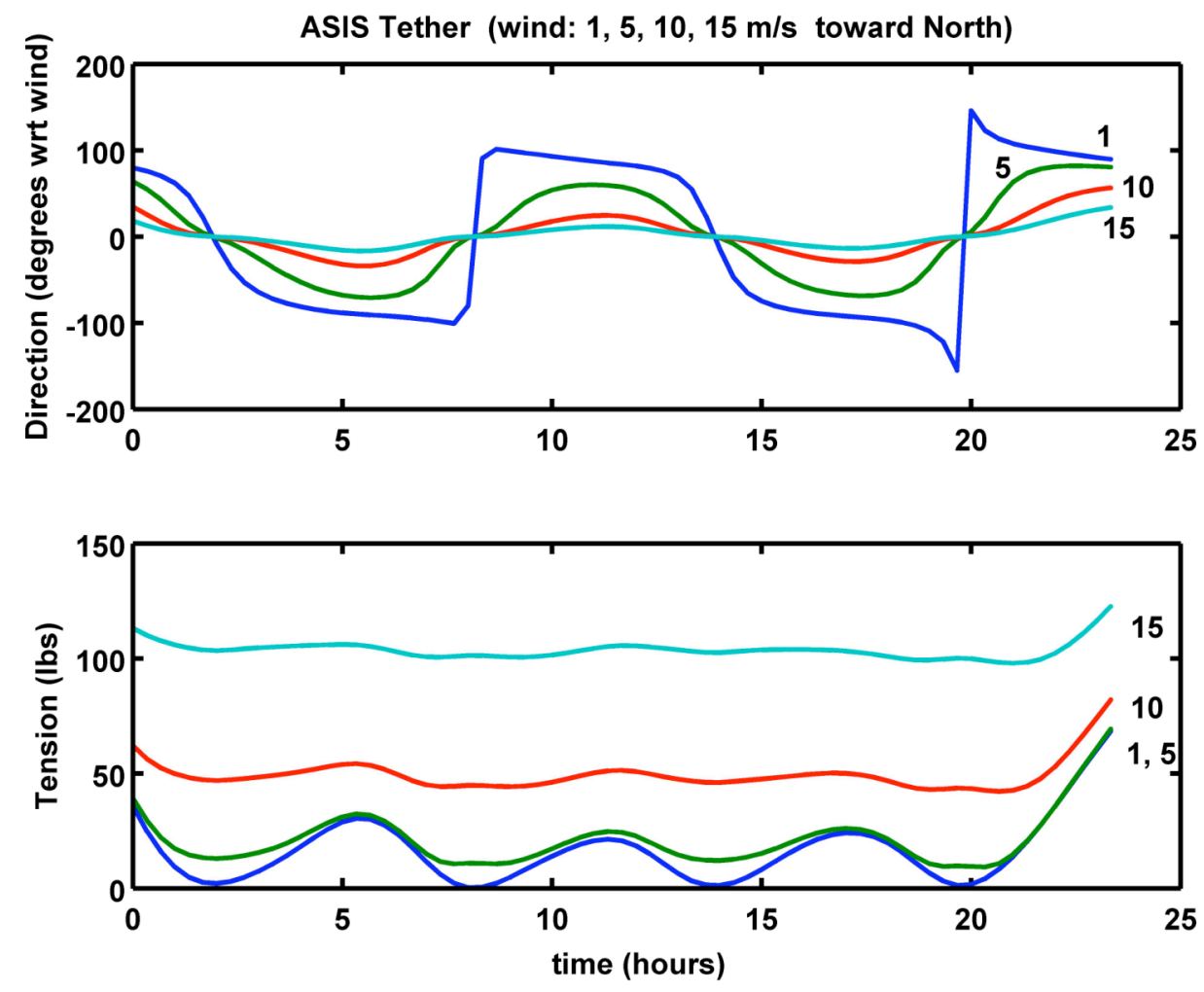

Figure 4.26 Direction of ASIS tether (top panel) and tether tension (bottom panel) for a given tidal cycle and winds of $1,5,10,15 \mathrm{~m} / \mathrm{s}$.

\begin{tabular}{|c|c|c|c|c|}
\hline Wind speed (m/s) & 1 & 5 & 10 & 15 \\
\hline Wind drag (lbs) & 0.452 & 11.3 & 45.2 & 102 \\
\hline Mean tension (lbs) & 16.1 & 21.0 & 49.3 & 104 \\
\hline \% error & 97 & 46 & 8.3 & 1.9 \\
\hline
\end{tabular}

Table 4.6 Tether tension for different wind speeds. 


\section{Chapter 5}

\section{Sodar Performance on ASIS and Conclusions}

\subsection{Overview}

As discussed in section 2.4, sodar performance mainly depends on reduction in the signal-to-noise ratio (SNR) due to the motion-induced decrease in scattering volume overlap between the transmit and receive beams. Since typical SNR values over the ocean are not well known, we focus here on the ratio $S N R / S N R_{0}$, where SNR is the received SNR, and $S N R_{0}$ is the SNR that would have been received in the absence of motion. In this chapter, we compute the probability of the reduction in SNR due to: [1] heave (z), [2] surge (x), and [3] pitch ( $\theta)$, using wave measurements taken during 2005 at the MVCO. We choose to study these three motions because they are the generic building blocks of more complex motions.

\subsection{SNR probability}

To analyze the performance of the sodar on a buoy, we need the probability that the SNR ratio exceeds a value, $\rho$, where $0 \leq \rho \leq 1$, for a given sea state. We compute this conditional probability separately for vertical and horizontal translations (i.e. "heave" and "surge") and tilt (i.e. "pitch") as a function of sea state. Averaging this conditional probability over the probability of the occurrence of various sea states gives the data availability for a specified reduction in SNR.

To illustrate how the conditional probability is determined, we consider the case of a $\mathrm{z}$ translation ("heave"). The same argument applies without change to the other motions. We are interested in the probability density function (pdf) of a change in buoy height, $z$, during a time $\Delta \mathrm{t}$. In appendix $\mathrm{G}$, we argue that the pdf is Gaussian, and hence can be written as

$$
p_{z}(z)=\frac{1}{\sigma \sqrt{2 \pi}} e^{-z^{2} / 2 \sigma^{2}}
$$


where the width, $\sigma$, depends on the sea state and the time difference $\Delta \mathrm{t}$. The probability that $S N R / S N R_{0}>\rho$ is

$$
P_{z}(\rho \mid \sigma) \equiv P_{z}\left(\operatorname{SNR} / S N R_{0}>\rho\right)=\int_{0}^{z(\rho)} p_{z}(z) d z / \int_{0}^{\Delta R} p_{z}(z) d z=\operatorname{erf}\left(\frac{z(\rho)}{\sigma_{z} \sqrt{2}}\right) / \operatorname{erf}\left(\frac{\Delta R}{\sigma_{z} \sqrt{2}}\right)
$$

where $\operatorname{erf}(y)$ is the "error function" (note that is has the property $\operatorname{erf}(y \rightarrow \infty) \rightarrow 1$ and $\operatorname{erf}(y \rightarrow 0) \rightarrow 0)$, and we have taken into account the fact that $\rho=0$ for $z$ outside the interval $|z| \leq \Delta R$ (here $\Delta R$ is the radial extent of the range cell). The SNR ratio, $\rho$, can be determined as a function of $\mathrm{z}$ from an analysis of buoy motion. Inverting this to get $z(\rho)$ permits evaluation of the probability $\mathrm{P}(\rho \mid \sigma)$. The form of the probability in (5.2) applies as well to surge and tilt (i.e. motion in $\mathrm{x}$ and $\theta$ ). However, what must be determined in each case are the functions $z(\rho), x(\rho)$ and $\theta(\rho)$, and the widths $\sigma_{z}, \sigma_{x}$ and $\sigma_{\theta}$. We address this in the next two sections.

\subsection{Overlap percentages}

Although a general motion of the sodar platform results from the combination of motion in all six degrees-of-freedom, it is useful for conceptual purposes to consider how the three basic motions - heave, surge and pitch - separately affect the transmit/receive (T/R) volume overlap for a vertically-oriented beam. The estimates from the analysis of this simple geometry provide order-of-magnitude bounds appropriate for both slanted beams and more complex motions. We discuss these three motions in turn below. In all cases we assume that the SNR ratio is equal to the fractional change, $\Delta V / V$, in the $\mathrm{T} / \mathrm{R}$ volume overlap, and hence use these two terms interchangeably.

\section{[1] Heave}

If the sodar heaves an amount $\mathrm{z}$ during the round-trip travel time $(\Delta t=2 R / c$, where $c$ is the sound speed) to a sample volume at range $R$, then the fractional T/R volume overlap (shown in Figure 5.1) is approximately given by

$$
\rho(z) \sim 1-\frac{|z|}{\Delta R}
$$


where $\Delta R$ is the radial extent of the sample volume. This expression ignores the curvature of the sample volume, and models it as a rectangular disk of area $A$ and height $\Delta R$. For the narrow beams under consideration here $\left(6 \mathrm{~dB}\right.$ fwhm $\left.\sim 10^{\circ}\right)$ this is a reasonable approximation. Equation 5.3 is inverted to get $\mathrm{z}(\rho)$

$$
\frac{|z(\rho)|}{\sigma \sqrt{2}}=\frac{\Delta R}{\sigma \sqrt{2}}(1-\rho) .
$$

Substituting this expression into (5.2) gives the probability $\mathrm{P}(\rho \mid \sigma)$.

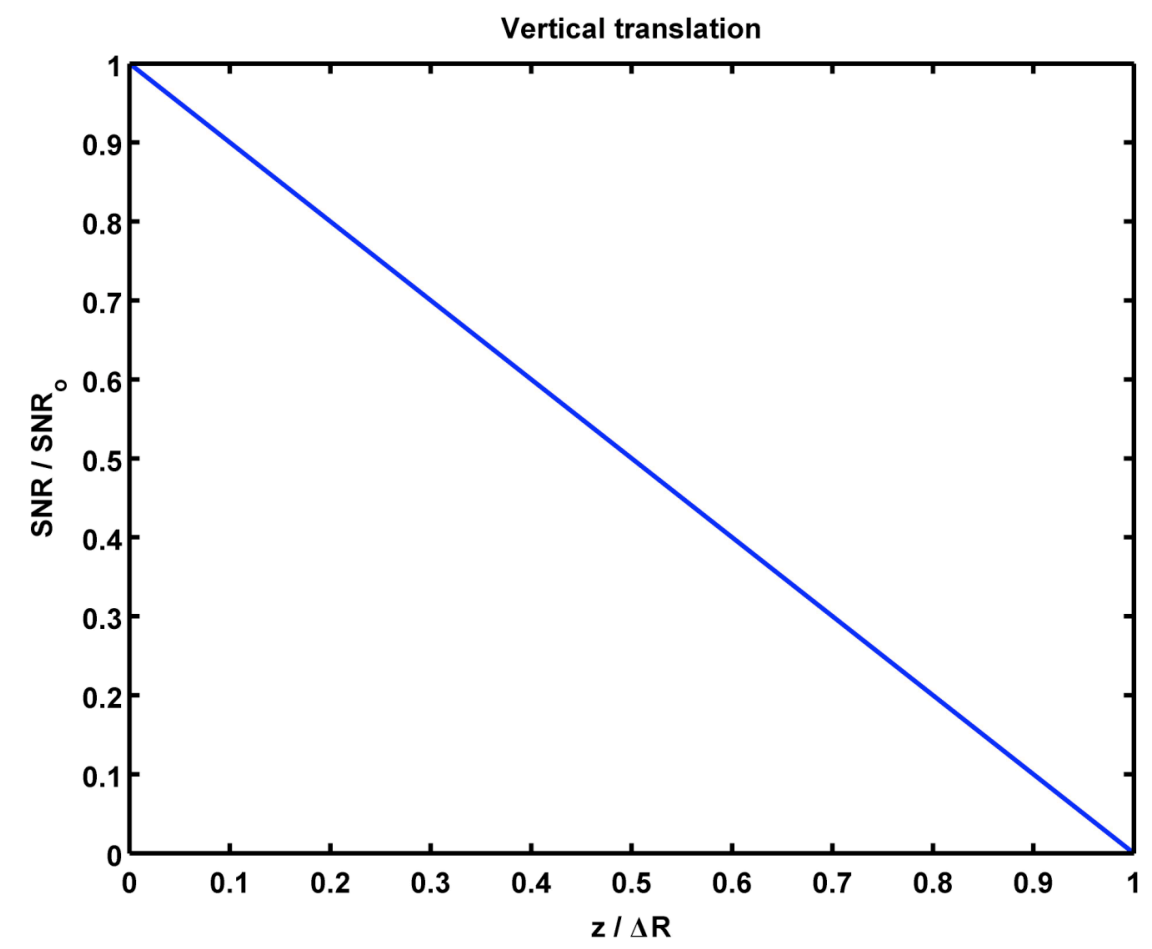

Figure 5.1 Heave volume fractional overlap (or, equivalently, SNR ratio).

\section{[2] Surge}

We compute the fractional volume overlap due to surge by carrying out a Monte Carlo integration of the overlap of the main lobe of two translated beam patterns. In order to suppress the effect of sidelobes, we have set the intensity distributions outside the main 
peak to zero (i.e. for values $|\theta| \geq 5.5^{\circ}$ ). Since the diameter, $d X$, of a range cell increases with range, the fractional overlap in surge depends on the radial distance to the range cell. The results of this calculation are shown in Figure 5.2 for a number of ranges.

The curves in Figure 5.2 collapse approximately to a single curve when plotted versus the "fractional surge", $X / d X$ (Figure 5.3).

The dashed line in Figure 5.3 shows the model

$$
\frac{|X|}{d X}=1-\rho
$$

Although this model slightly over-predicts our Monte Carlo results, the latter are an under-prediction of the true overlap due to the sharp cutoff imposed to restrict the calculation to the main lobe. As a result, we will use (5.5) in our calculation of the probability $P_{x}\left(\rho \mid \sigma_{x}\right)$. 


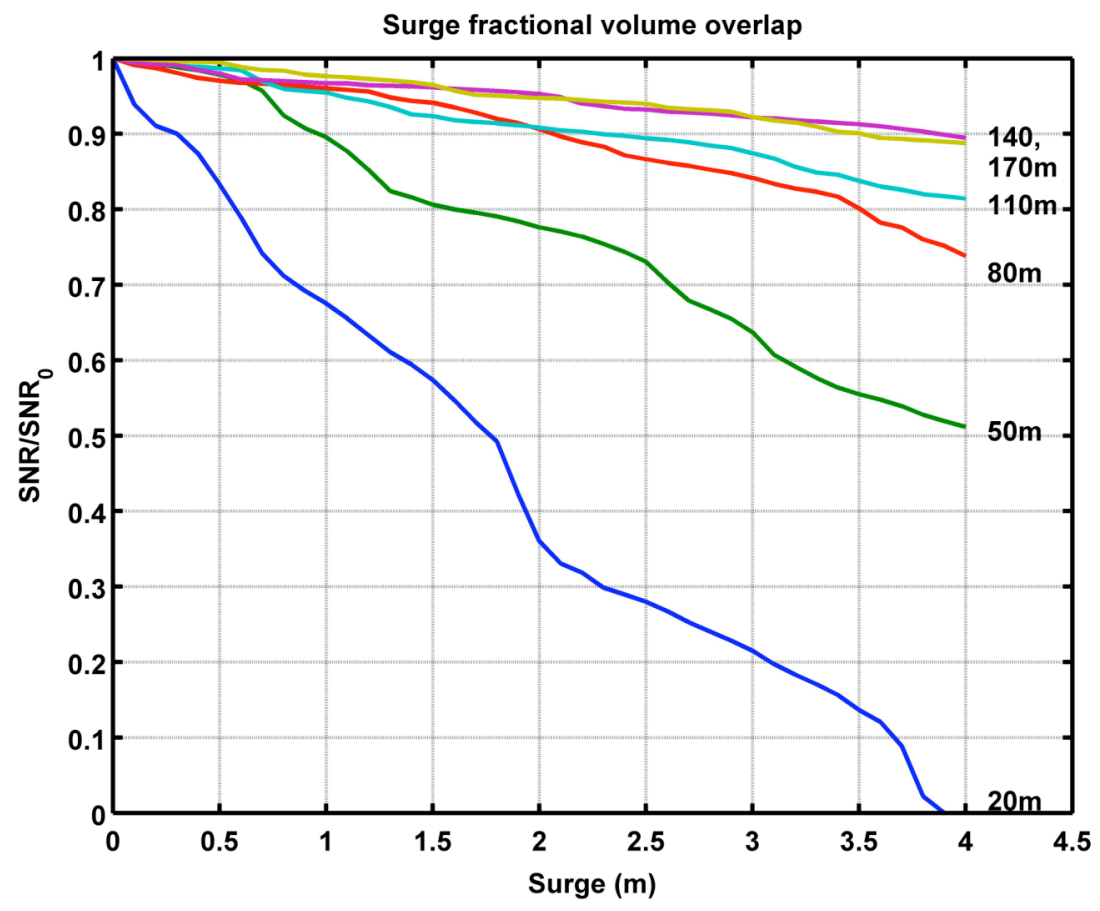

Figure 5.2 Surge fractional volume overlap for different range cells (labeled next to each curve).

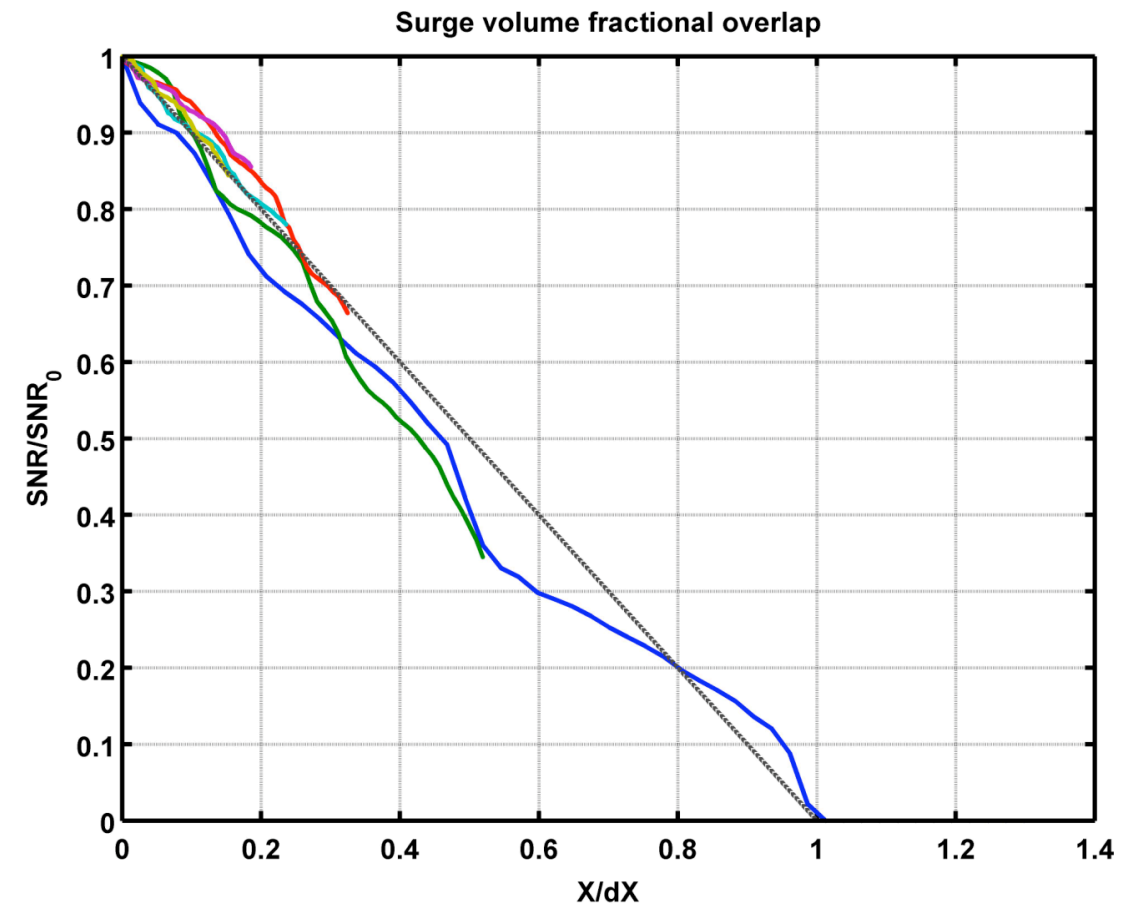

Figure 5.3 Surge volume fractional overlap versus $\mathrm{X} / d \mathrm{X}$. 


\section{[3] Pitch}

Similarly to surge, the degradation in SNR due to pitch is computed using a numerical Monte Carlo integration. Two beam patterns are created, one tilted with respect to the other. A uniform distribution of random points are selected in the range $0 \leq \phi \leq 2 \pi$, $0 \leq \theta \leq \pi / 2$, where $\theta$ and $\phi$ are the spherical polar and azimuthal angles, respectively. The result for tilts in a plane containing the sidelobes is shown in Figure 5.4.

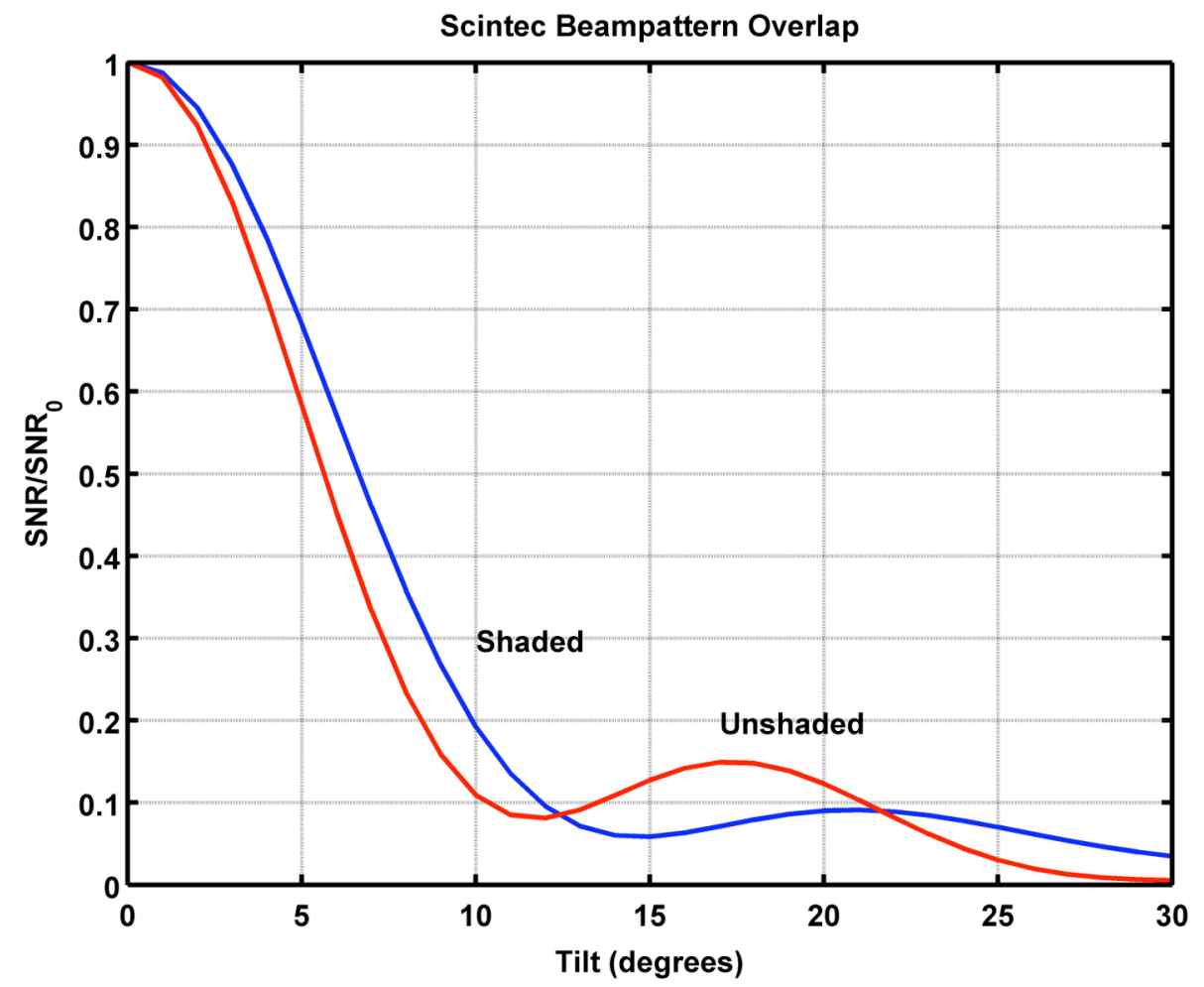

Figure 5.4 Scintec SFAS vertical beam pattern degradation in SNR due to pitch for the shaded and unshaded case.

Because this leads to a multi-valued function $\theta(\rho)$, we make the same simplification used in the case of surge by restricting $0 \leq \theta \leq \theta_{c}$. As before, we choose $\theta_{c}=5.5^{\circ}$ in order to include only the Monte Carlo main lobe of the unshaded T/R intensities. Figure 2.6 shows that the main lobe is azimuthally symmetric, so that this restricted calculation applies to a tilt in any direction. However, due to the sidelobe structure, we expect that it slightly under-predicts the volume overlap for tilts in the planes containing the sidelobes. The results are shown in Figure 5.5. 


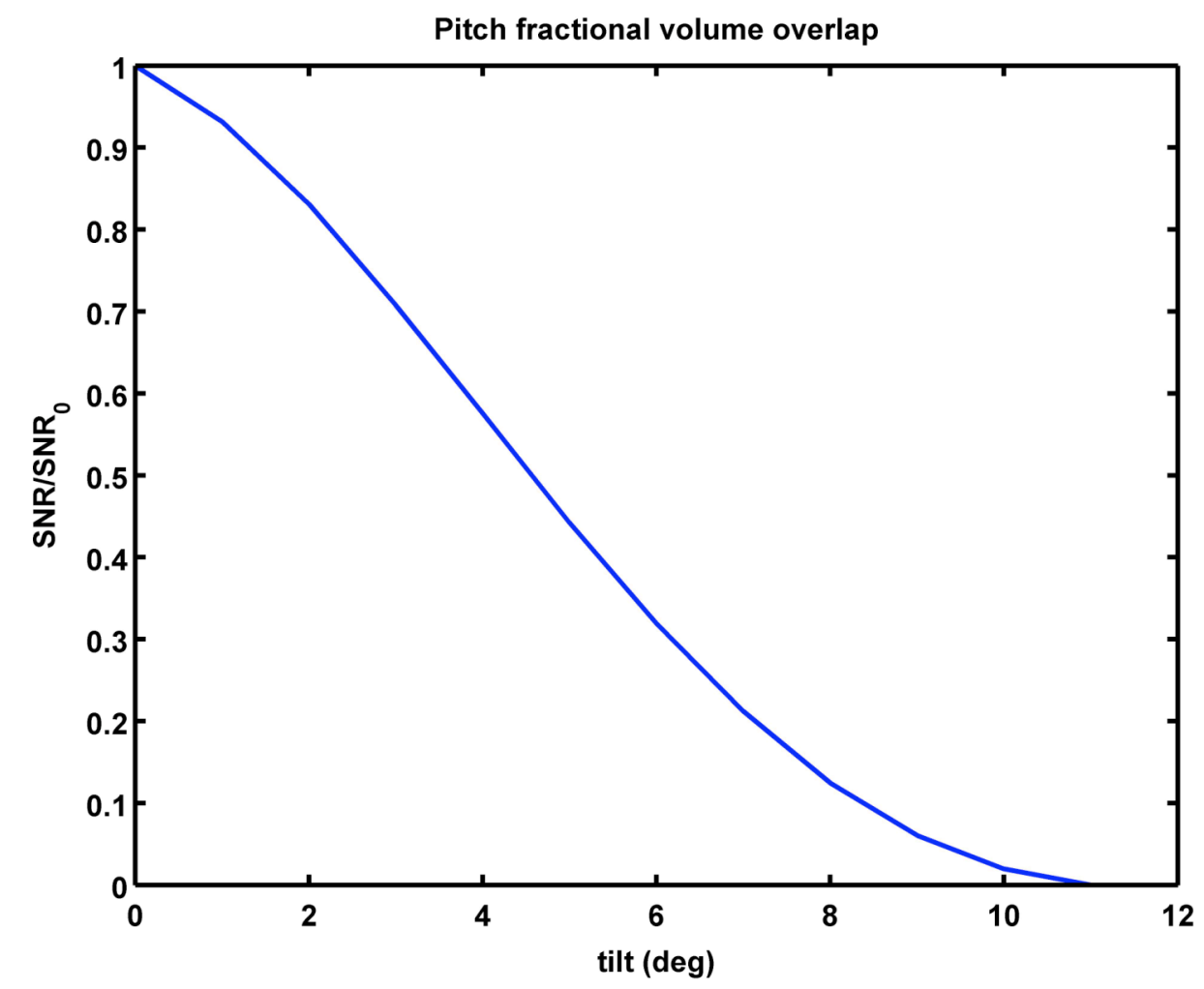

Figure 5.5 Pitch volume fractional overlap using only the main lobe of the unshaded beam pattern.

\subsection{Calculation of the pdf widths $\sigma$}

In this section we compute the standard deviations, $\sigma$, of the pdfs of for heave, surge and pitch increments for 3-meter discus and ASIS buoys as a function of sea state and time lag $\Delta$ t. We make use of observations taken during 2005 of the wave height spectrum measured using the ADCP at the $12 \mathrm{~m} \mathrm{MVCO}$ node.

A power spectrum was estimated for 22,689 20-minute bursts. These were averaged into $20 \times 21$ bins in mean frequency and significant wave height. Each averaged spectrum was used to synthesize a random time series of wave height, $h(t)$. Defining 20 uniformlyspaced time lags, $\Delta \mathrm{t}$, from $50 \mathrm{~ms}$ to $1 \mathrm{~s}$ (corresponding to sodar ranges of $8-170 \mathrm{~m}$ ), we computed time series of heave increments $d h=h(t+\Delta t)-h(t)$ for each sea state. The pdf of each $d h$ has the form of a zero-mean Gaussian distribution, where $\sigma$ specifies the 
width. The overall the behavior of $\sigma$ is found to be very close to linear in $\Delta t$ (details are given in Appendix G).

Spectra of wave slope were obtained from the height spectra by multiplying the spectral value in each frequency band by $k^{2}$, where $k(f)$ is the wavenumber corresponding to frequency $f$. For computations involving the ASIS, the spectra were further multiplied by the appropriate ASIS transfer function (described in section 4.3). In computations pertaining to the 3-meter discus buoy, we assumed that the 3-meter discus buoy follows the sea surface in heave, surge and pitch for all waves with frequencies below $0.5 \mathrm{~Hz}$, and therefore used a response function of unity over the entire measured spectrum.

Figures 5.6-5.11 show the computed $\sigma$ 's for heave, surge and pitch increments over $\Delta \mathrm{t}=1$ second for the 3-meter discus and ASIS buoys. The $\sigma$ 's for shorter time intervals would be proportionately smaller. Sea states are assumed to be characterized by the significant wave height, $H_{S}$, and the mean frequency, $F_{P}$ (computed as the normalized first moment of the wave height spectrum). 


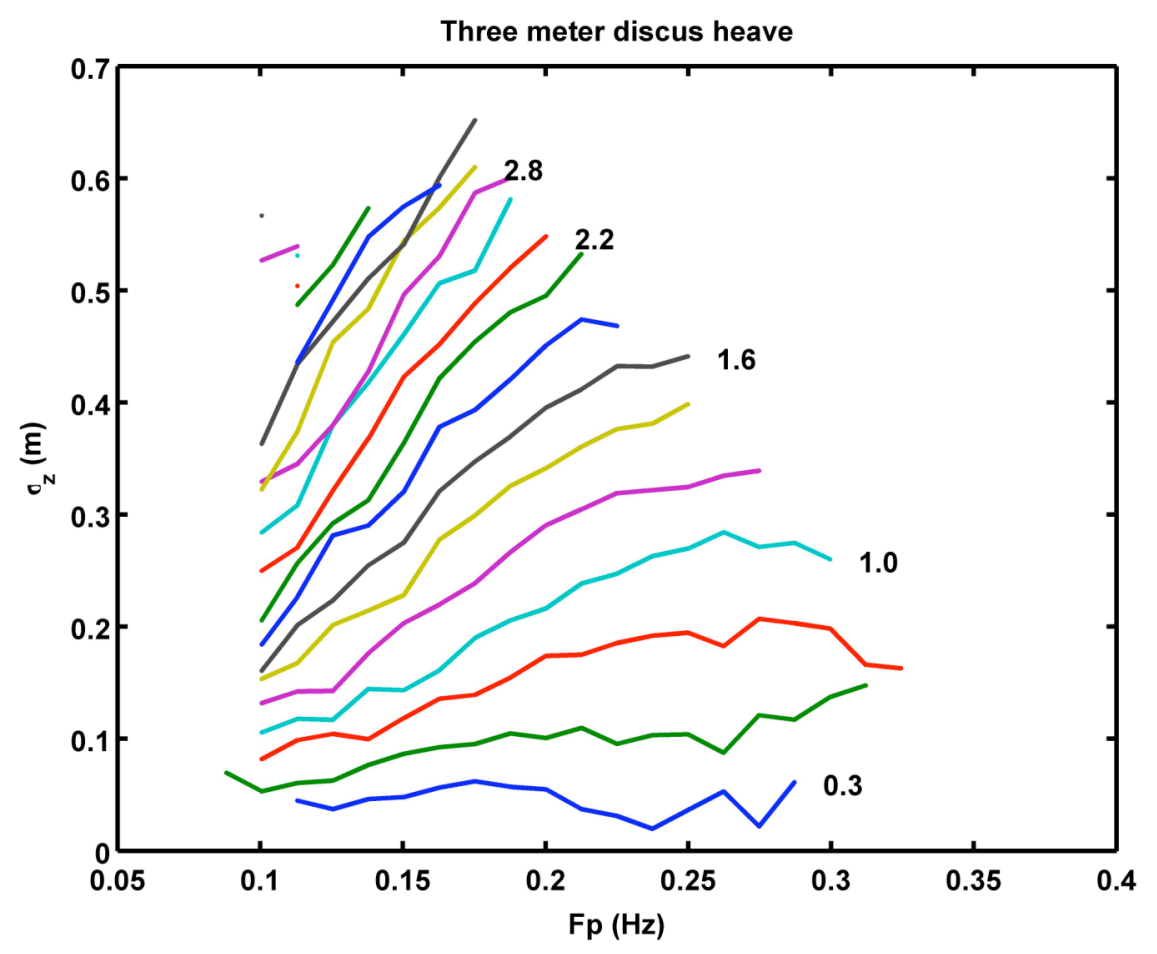

Figure 5.6 The standard deviation, $\sigma_{\mathrm{z}}$, of the increment in heave over 1 second for a 3-meter discus buoy. Each curve shows the dependence of $\sigma_{z}$ on the mean wave frequency for a fixed interval of significant height. The latter (in meters) appears to the right of the curves. 


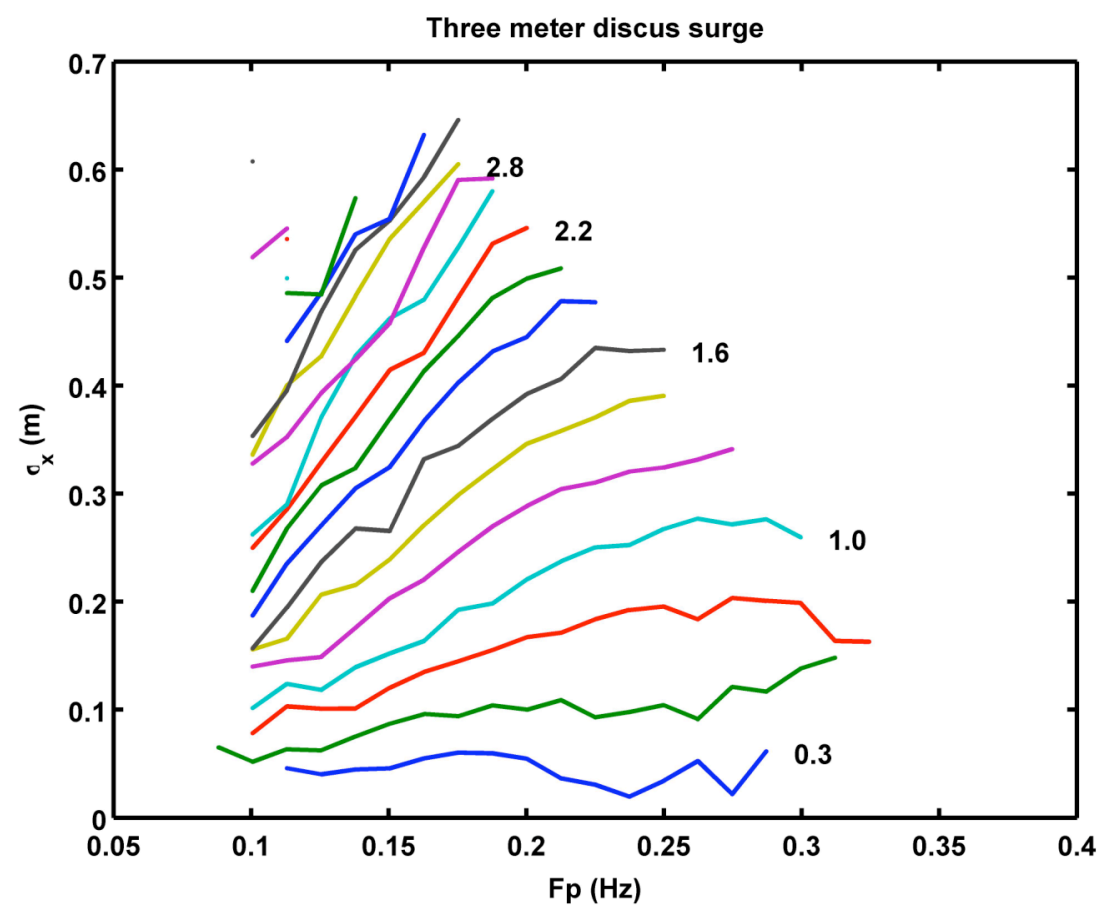

Figure 5.7 The standard deviation, $\sigma_{\mathrm{x}}$, of the surge increment over $\Delta t=1$ second for a 3-meter discus buoy.

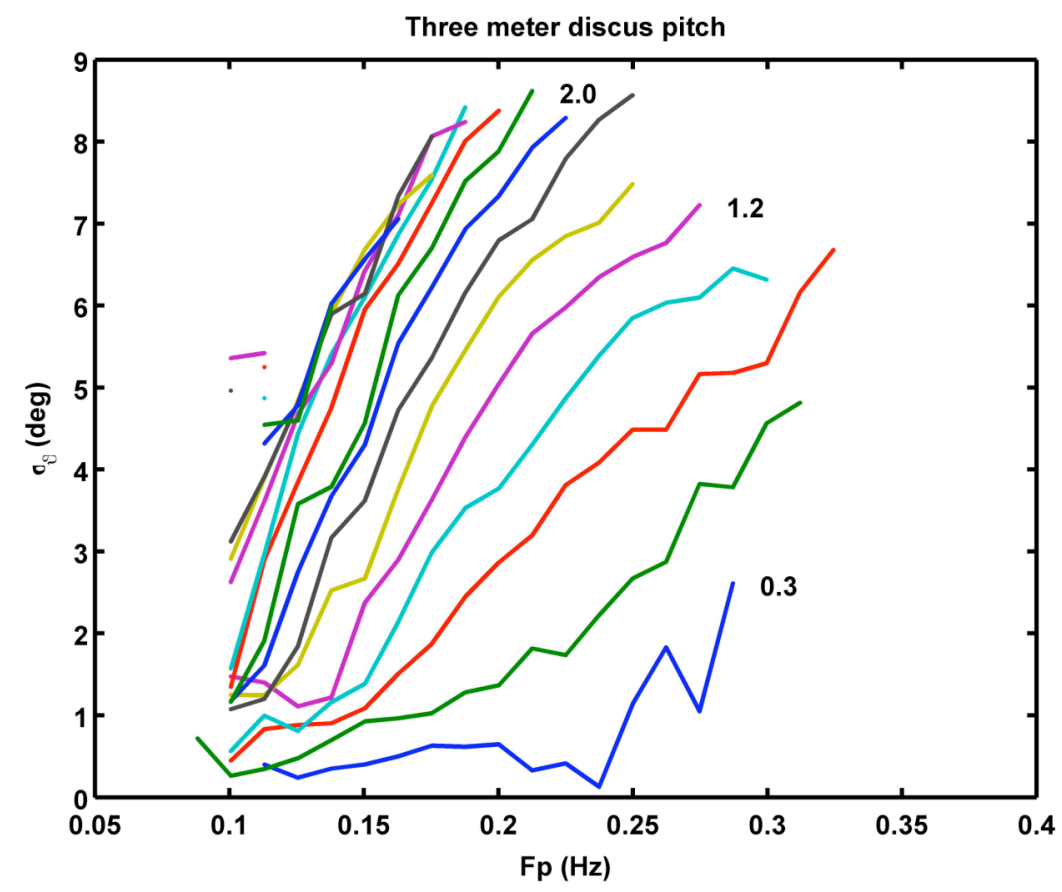

Figure 5.8 The standard deviation, $\sigma_{\theta}$, of the pitch increment over $\Delta t=1$ second for a 3-meter discus buoy. 


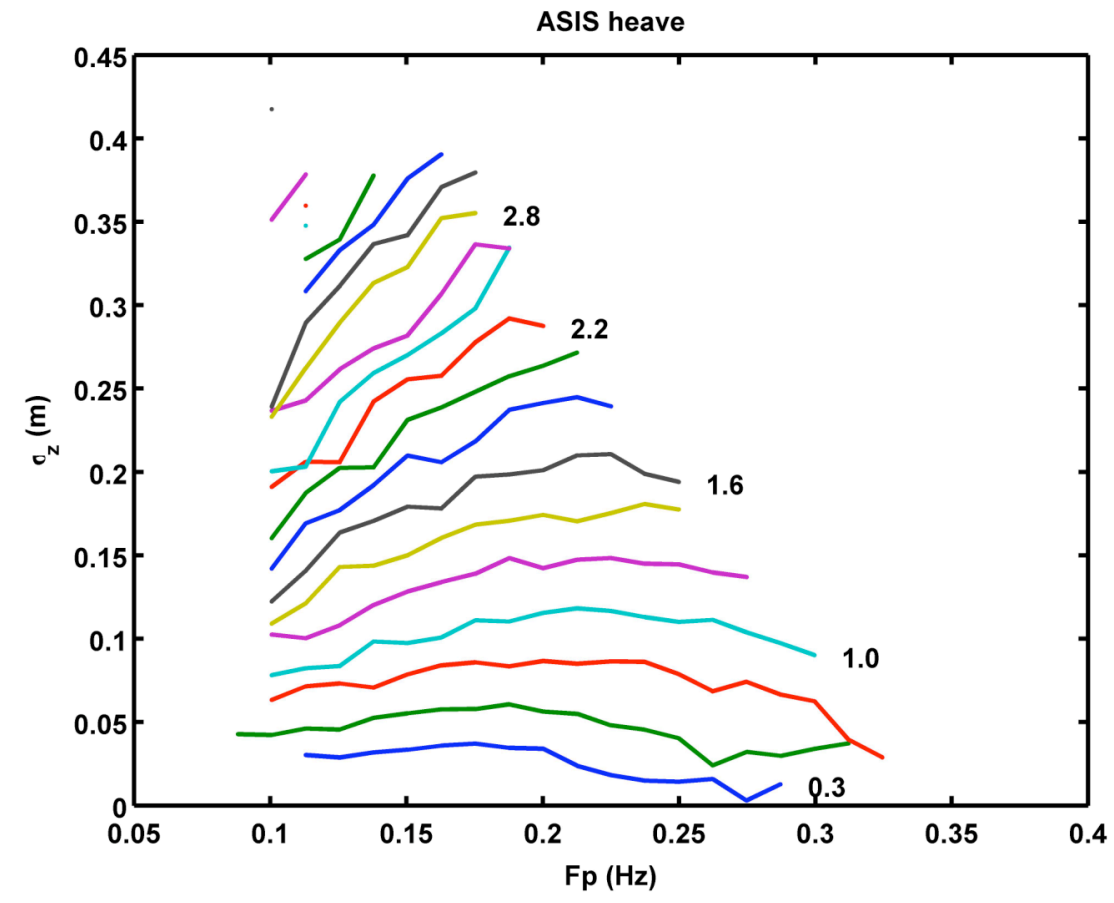

Figure 5.9 The standard deviation, $\sigma_{z}$, of the heave increment over $\Delta t=1$ second for the ASIS buoy.

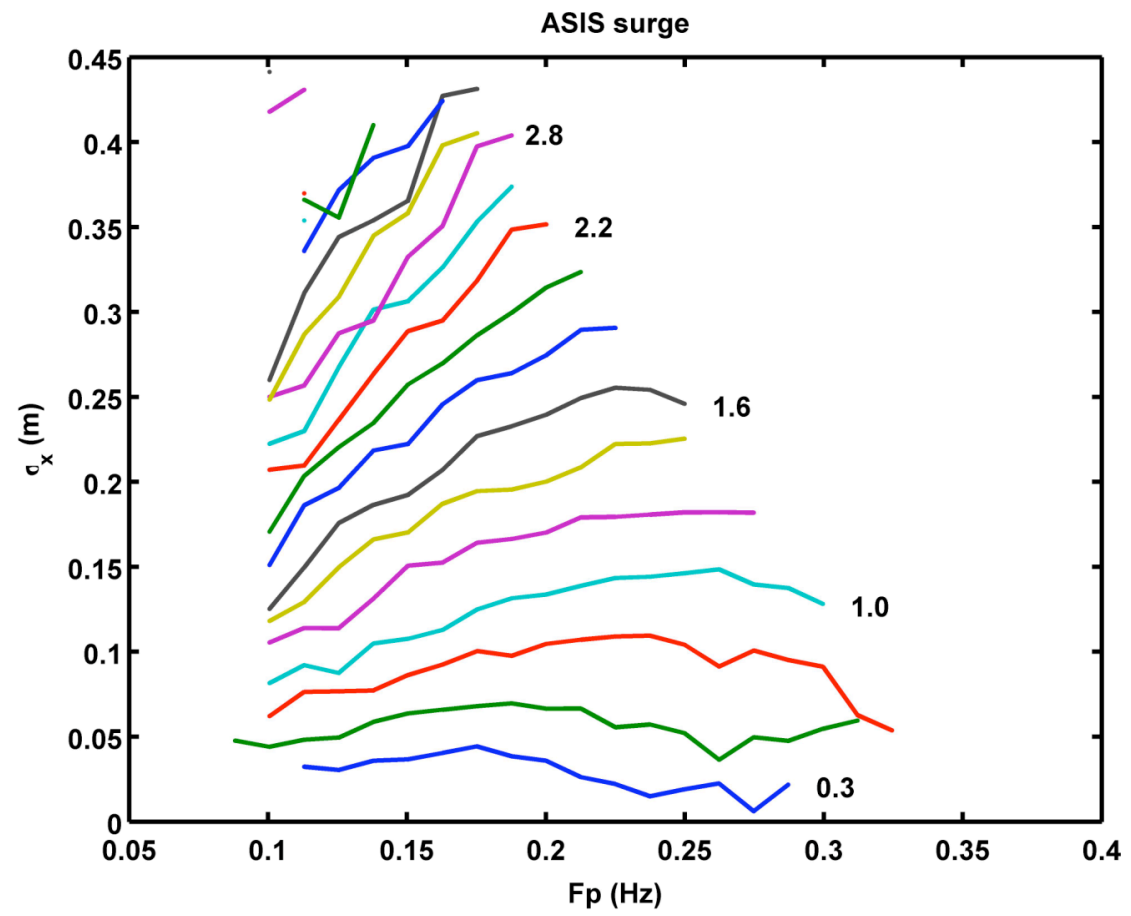

Figure 5.10 The standard deviation, $\sigma_{x}$, of the surge increment over $\Delta t=1$ second for the ASIS buoy. 


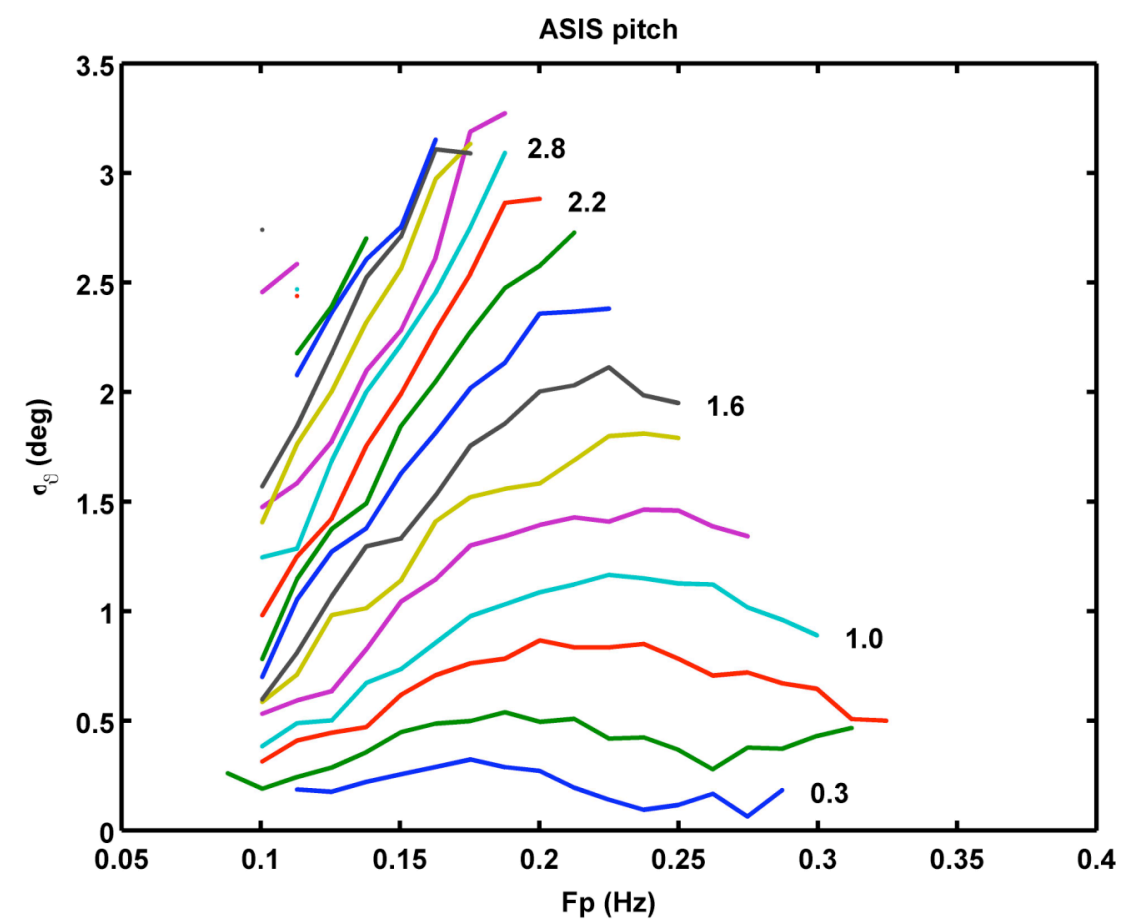

Figure 5.11 The standard deviation, $\sigma_{\theta}$, of the pitch increment over $\Delta \mathrm{t}=1$ second for an ASIS buoy.

\subsection{Conditional exceedance probability}

The expected data availability from the sodar in a particular sea state depends on the probability that the received SNR is not too severely reduced. Hence we compute the probability for $S N R / S N R_{0}$ to exceed a particular value $\rho$. To do this we use equation 5.2 along with $z(\rho), x(\rho), \theta(\rho)$ (see section 5.3) to obtain curves of the conditional probability $P(\rho \mid \sigma)$. Plots of these curves for vertical translation and tilt are shown in Figures 5.12 and 5.13, respectively. Figure 5.14 shows a similar plot for the surge calculation, but in this case the curves represent $\sigma / d r$, where $d r$ is the diameter of the main lobe of the sodar beam pattern. Minimum and maximum values of $\sigma_{x}, \sigma_{z}$, and $\sigma_{\theta}$ for both the 3-meter discus and ASIS buoys are given in Table 5.1. 


\begin{tabular}{|c|c|c|c|c|}
\hline & $\min \sigma(\Delta t=1 s)$ & $\max \sigma(\Delta t=1 s)$ & min rms & $\max r m s$ \\
\hline 3-m discus $z$ (m) & 0.0197 & 0.652 & 0.0106 & 1.10 \\
\hline $\mathbf{x}(\mathrm{m})$ & 0.0195 & 0.646 & 0.0106 & 1.10 \\
\hline$\theta$ (deg) & 0.128 & 8.62 & 0.151 & 5.94 \\
\hline ASIS z (m) & 0.00301 & 0.418 & 0.00146 & 0.887 \\
\hline $\mathbf{x}(\mathbf{m})$ & 0.00621 & 0.441 & 0.00302 & 0.922 \\
\hline$\theta$ (deg) & 0.0629 & 3.27 & 0.0306 & 3.67 \\
\hline
\end{tabular}

Table 5.1 Minimum and maximum $\sigma$ values for the 3-meter discus and ASIS buoys.

The values of $\sigma$ in Table 5.1 are all computed for $\Delta \mathrm{t}=1$ second and therefore represent upper-bounds for all sodar range cells below $170 \mathrm{~m}$. Note that the rms motion and $\sigma$ values for ASIS are generally smaller than those for the 3-meter discus.

The largest $\sigma_{z}$ and $\sigma_{x}$ values in 2005 for ASIS were 0.42 and $0.44 \mathrm{~m}$, respectively, corresponding to a significant wave height of roughly $4 \mathrm{~m}$. From Figures 5.12 and 5.14 we find that for $S N R / S N R_{0}>0.8$ the effect of heave and surge are likely to be negligible for both buoy's - even in high sea states maximum values of $\sigma_{\theta}$ for both buoys are considerably larger (3.2 and 8.6 for ASIS and the discus, respectively). Then, for example, if we require $S N R / S N R_{0}>0.7$, the data availability for the discus is around $35 \%$, where it is $70 \%$ for ASIS. We conclude that ASIS performs much better in high sea states than the 3-meter discus buoy. 


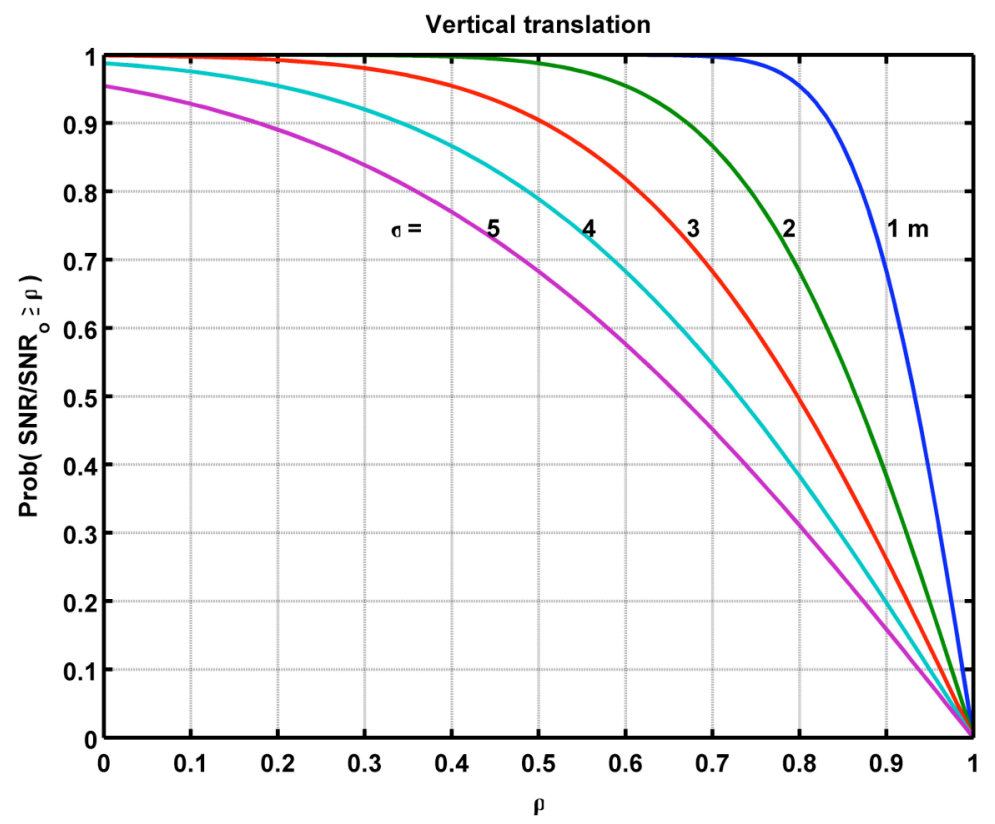

Figure 5.12 The probability that $\mathrm{SNR} / \mathrm{SNR}_{0} \geq \rho$ as a function of $\sigma$ for vertical translation. $\sigma$ 's are given for a range cell size $R=5 \mathbf{~ m}$.

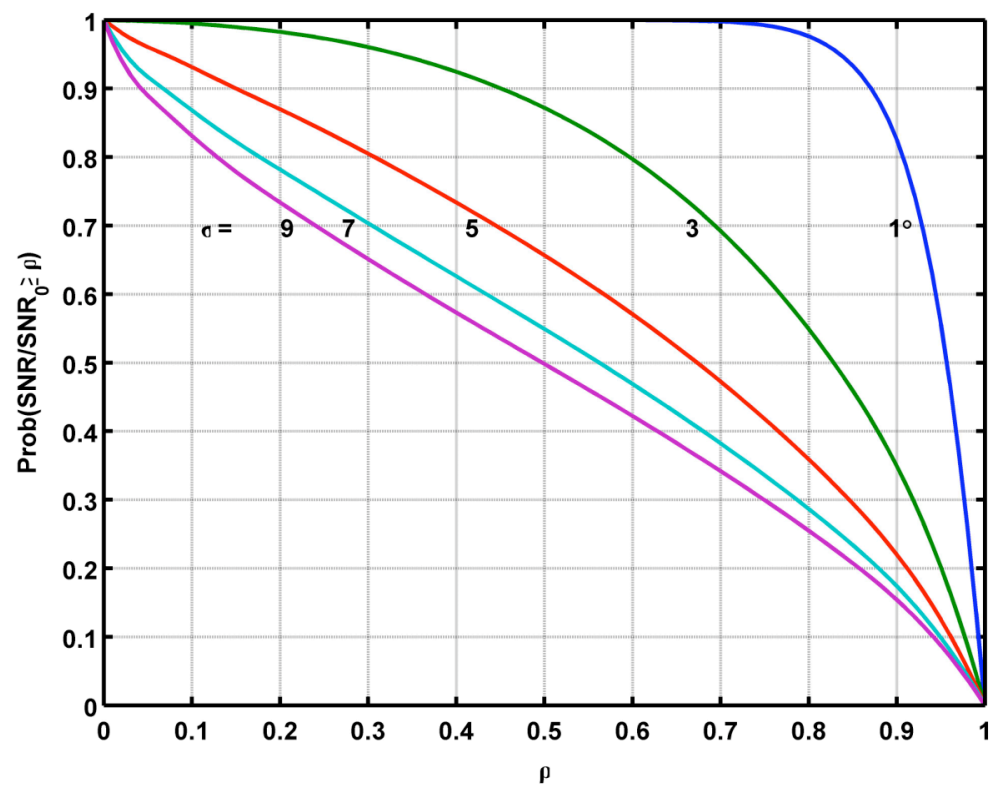

Figure 5.13 The probability that $\mathrm{SNR} / \mathrm{SNR}_{0} \geq \rho$ as a function of $\sigma$ for tilt. 


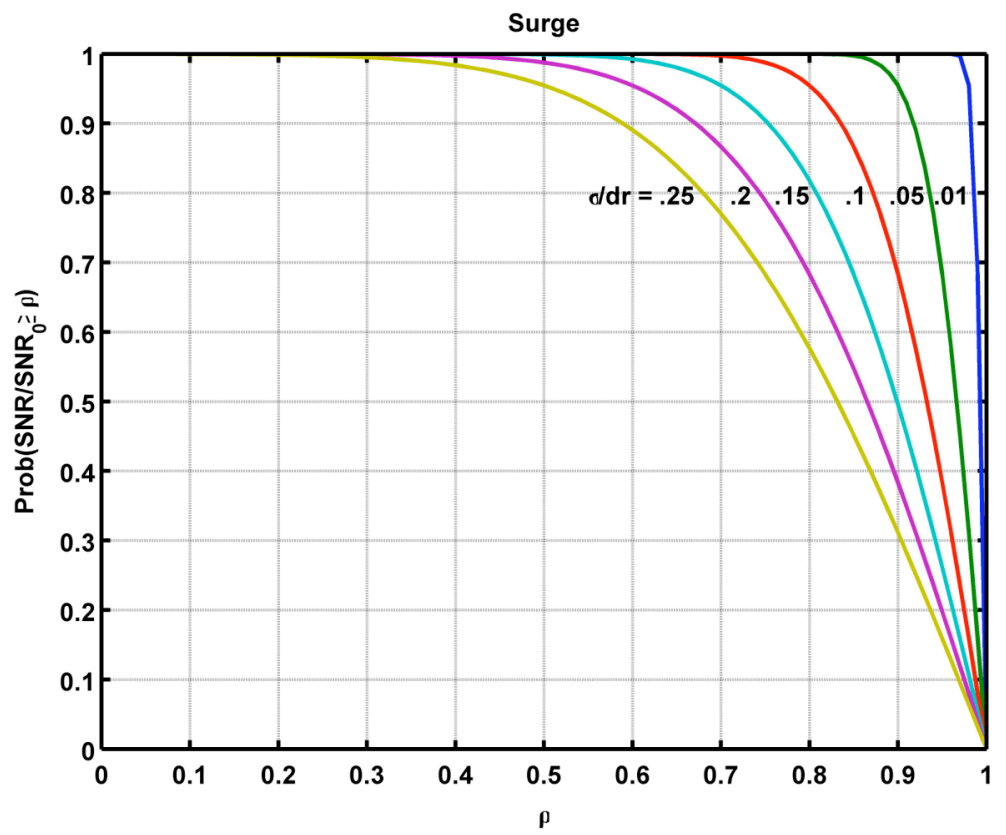

Figure 5.14 The probability that $\mathrm{SNR} / \mathrm{SNR}_{0} \geq \rho$ as a function of $\sigma / d r$ for surge. $d r$ is the diameter of the range cell.

\subsection{Yearly average data availability due to motion}

In this section, we compute the expected data availability over a year for a sodar mounted on both a 3-meter discus and ASIS that is moving in heave, surge and pitch. We do this by averaging the conditional probabilities $P(\rho \mid \sigma)$ for heave, pitch and surge over the probability for the observed occurrence of various sea states. Then

$$
\bar{P}(\rho \mid \Delta t)=\sum_{F_{p}, H_{S}} P_{S}\left(F_{P}, H_{S}\right) P(\rho \mid \sigma)
$$

where $\sigma=\sigma\left(F_{P}, H_{S}, \Delta t\right)$ and $P_{S}$ is the probability of a sea state lying in the bin centered on $\left(F_{P}, H_{S}\right)$. We compute $\sigma$ for a lag of $\Delta \mathrm{t}=1$ second since this is an upper bound on $\sigma$, and hence gives a lower bound on the percentage of data availability. Note, however, that whereas $P(\rho \mid \sigma)$ depends on $\Delta \mathrm{t}$ in the case of pitch and heave, for surge it is independent of $\Delta \mathrm{t}$ (or, equivalently, range) because the increase in $\sigma$ with range is compensated by a corresponding increase in the diameter of the range cell. This follows because (see section 5.3) 


$$
\frac{X(\rho)}{\sigma_{x} \sqrt{2}} \approx \frac{d r}{\sigma_{x} \sqrt{2}}(1-\rho)
$$

where $d r$ is the diameter of the range cell at distance $R$. But $d r \sim R$ and $\sigma_{x} \sim \Delta t \sim R$, so that their ratio is independent of $\Delta \mathrm{t}$.

Plots of the yearly averaged data availability for the three buoy motions seen by the 3meter discus and ASIS buoys are shown in Figures 5.15 - 5.17.

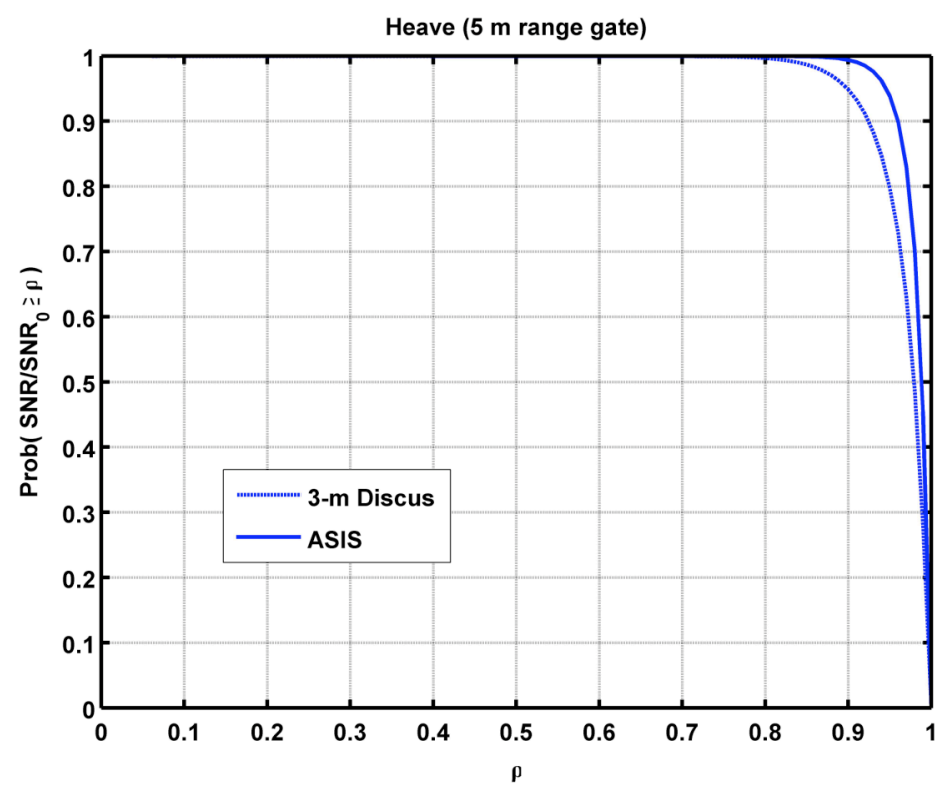

Figure 5.15 Yearly average data availability in heave for the 3-meter discus and ASIS buoys. Curves are for the range $R=170 \mathrm{~m}$ (i.e. $\Delta \mathrm{t}=1 \mathrm{~s}$ ) and hence give a lower-bound on the percentage of data availability. 


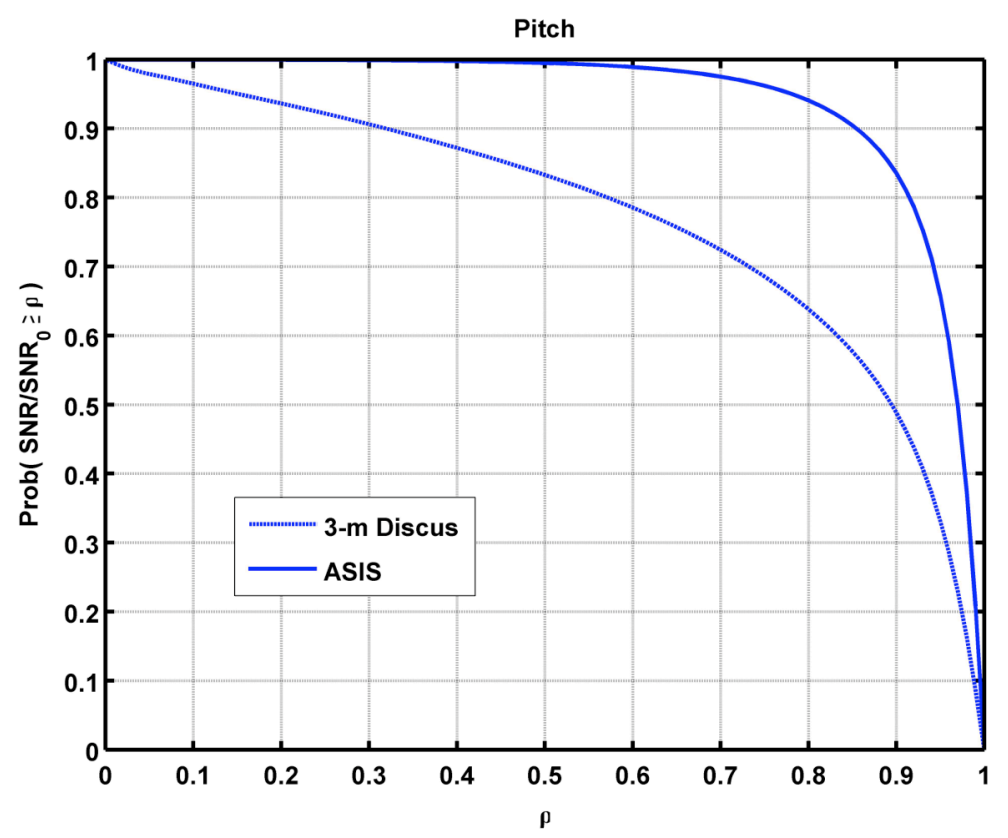

Figure 5.16 Yearly average probability for data availability in pitch for the 3-meter discus and ASIS buoys. Curves are for the range $R=170 \mathrm{~m}$.

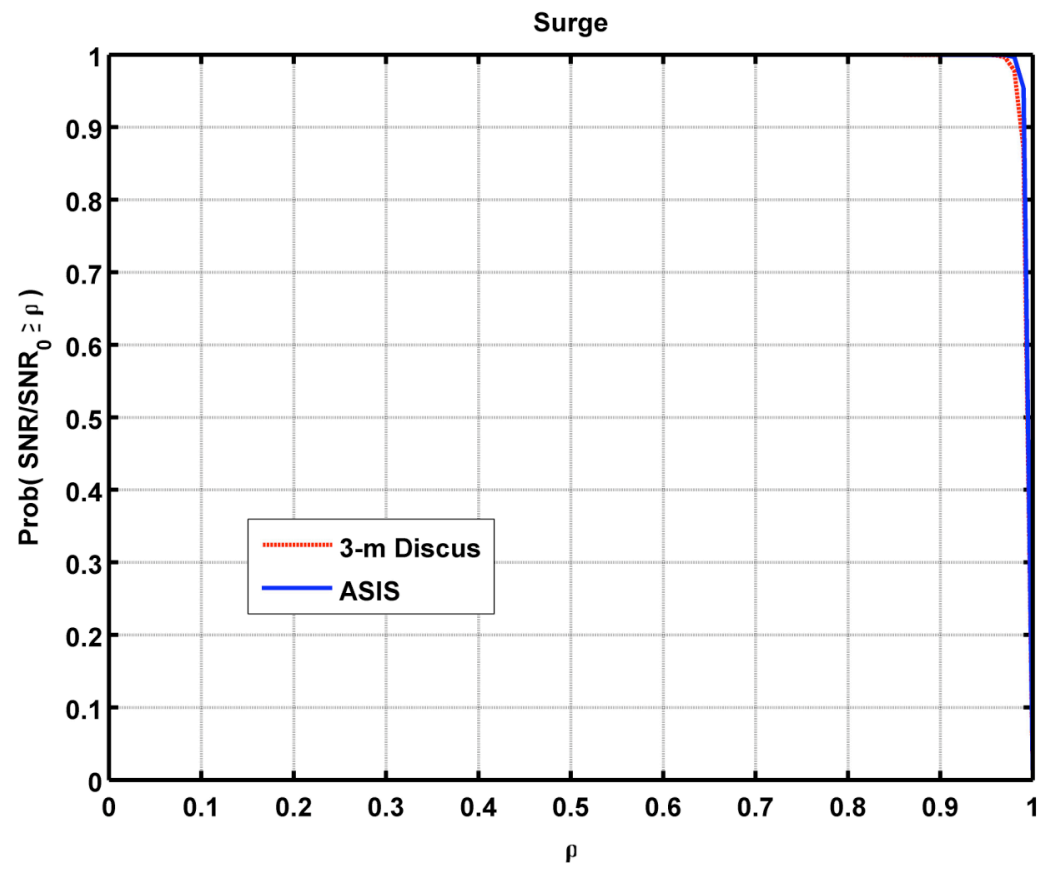

Figure 5.17 Yearly data availability in surge for the 3-meter discus and ASIS buoys. Curves are independent of range. 
Figures 5.18 and 5.19 summarize yearly average data availability in heave, pitch and surge for the ASIS and 3-meter discus buoys, respectively.

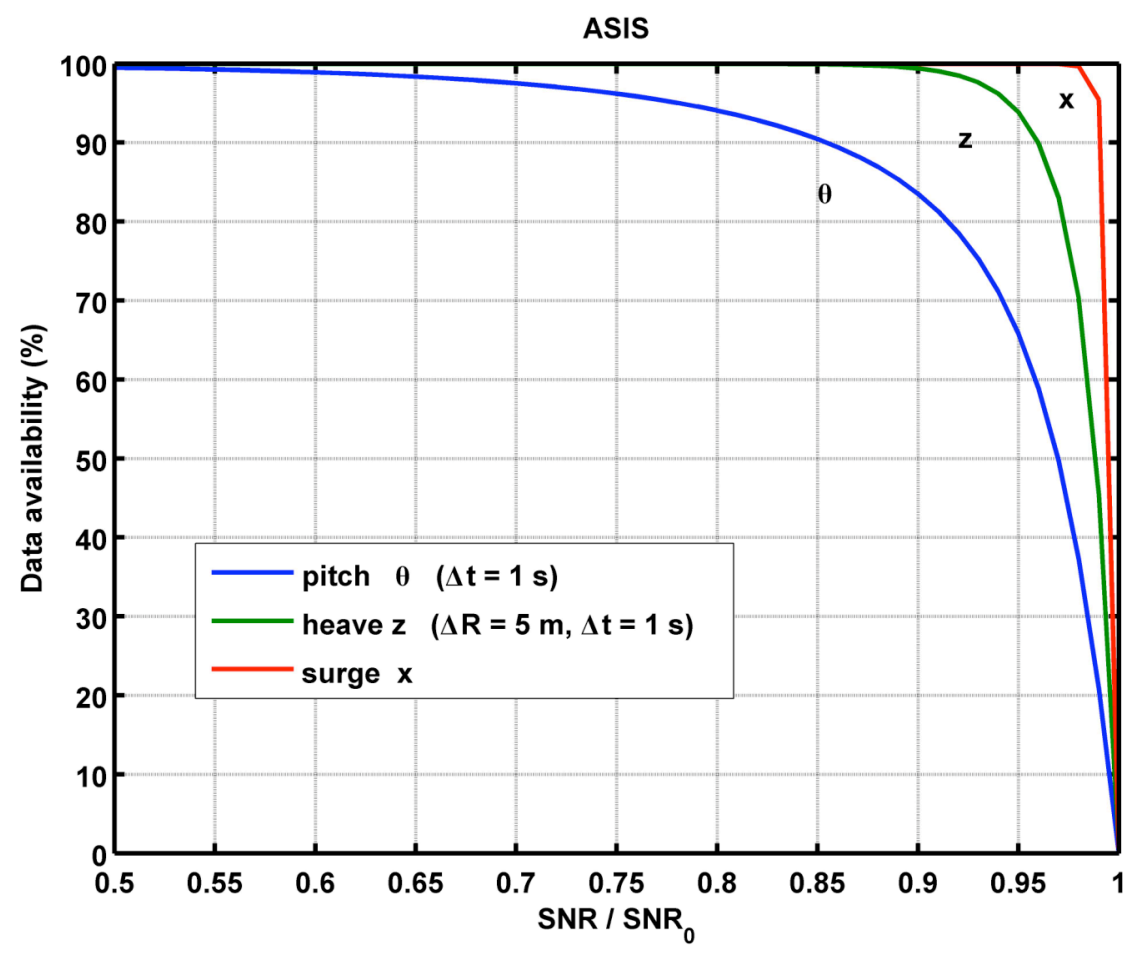

Figure 5.18 ASIS buoy \% data availability over 2005 for pitch, heave and surge. 


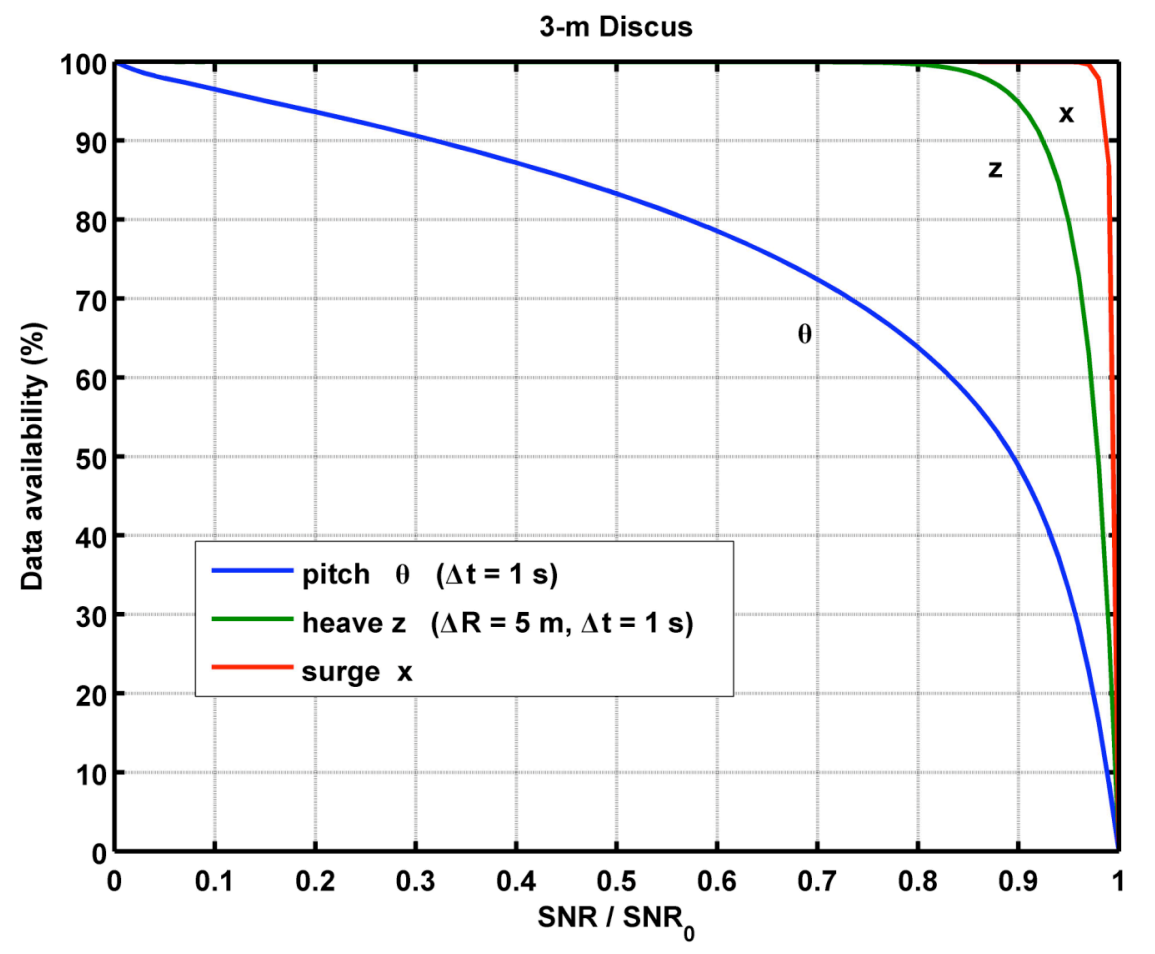

Figure 5.19 3-meter discus buoy \% data availability over 2005 for pitch, heave and surge.

\subsection{Conclusions}

We have analyzed the operation of Doppler sodar and concluded that the principal effect of platform motion is an increase in the variance of the Doppler frequency estimate due to a decrease in the received signal-to-noise ratio (SNR). The reduction in SNR is a consequence of a decrease in the spatial overlap between the transmit and receive beams due to the motion. Based on a full year of observations of wave height spectra taken at the MVCO 12-m node, we have developed quantitative predictions of the probability for the SNR (relative to the case of no motion) to exceed any value in the range 0 to 1 , as a function of sea state. These conditional probabilities were averaged over the observed probability of occurrence of the various sea states to predict a yearly data availability for a sodar deployed on either an ASIS or a 3-meter discus buoy (Figures 5.18 and 5.19). 
Our analysis predicts that ASIS will be an acceptable platform for sodar measurements at sea. The 3-meter discus buoy may also be an acceptable platform in terms of yearly data availability, but fails rapidly to provide a stable enough platform in high sea states.

SNR reduction due to heave and surge is negligible for both the ASIS and 3-meter discus buoys. For example, the yearly probability of data availability in heave is 80 and $90 \%$ for the 3-meter and ASIS buoys, respectively. Data availability for both buoys in heave is similar to that in surge.

Sodar performance is most heavily affected by buoy tilting due to wave forcing. ASIS is substantially less reactive to forcing in pitch than the 3-meter discus buoy. For example, ASIS has $90 \%$ data availability for a $15 \%$ decrease in SNR, while the 3-meter discus buoy gives about $60 \%$ data availability.

In fact, the data availability models for the 3-meter discus buoy most likely over-estimate sodar performance. In this analysis, the 3-meter discus buoy was assumed to follow the slope of the sea surface, when in reality, discus buoys are sensitive to mooring dynamics. During periods of strong forcing by winds and currents, their moorings can become taut causing the buoy to experience a more erratic motion. In contrast, ASIS buoys are attached laterally to a surface mooring by a buoyant tether, and hence the effect of the mooring on the buoy motion is substantially reduced. Also, because ASIS has a large $(\sim 6 \mathrm{~m}) \mathrm{draft}$, it can be ballasted to increase its righting moment, and hence further reduce its response in pitch and roll.

Our results strongly suggest that the combination of a sodar deployed on an ASIS buoy is a feasible and practical technique for profiling winds over the lower several hundred meters of the marine atmospheric boundary layer. 


\section{References}

Barthelmie, R.J., L. Folkerts, F.T.Ormel, P.Sanderhoff, P.J.Eecen, O.Stobbe, and N.M.Nielsen (2003): Offshore wind turbine wakes measured by sodar. Journal of Atmospheric and Oceanic Technology., 20, 466-477.

Brown, Robert G. and Patrick Y.C. Hwang (1997): Introduction to random signals and applied Kalman filtering, $3^{\text {rd }}$ ed., J. Wiley.

Clay, Clarence S., Herman Medwin (1977): Acoustical Oceanography, J. Wiley.

Coelingh, J., L. Folkerts, G. Wiegerinck, and E. van Zuylen (2000): Study of the offshore wind climate using a sodar. Offshore Wind Energy in Mediterranean and other European Seas, ATENA, 61-70.

Crescenti, G. H. (1997): A look back on two decades of Doppler sodar comparison studies. Bull. Amer. Meteor. Soc., 78, 651-673.

Donelan, M.A., J. Hamilton and W.H. Hui (1985): Directional spectra of wind-generated waves. Phil. Trans. Roy. Soc. Lond., 315, 509-562.

Fairall, C.W., A.B. White, J.B. Edson, and J.E. Hare (1997): Integrated shipboard measurements of the marine boundary layer. J. Atmos. and Oceanic Technol., 14, 338359.

Graber, Hans C., Eugene A. Terray, Mark A. Donelan, William M. Drennan, John C. Van Leer and Donald B. Peters (2000): ASIS-A New Air-Sea Interaction Spar Buoy: Design and Performance at Sea. J. Atmos. and Oceanic Technol., 17, 708-720.

Jørgensen, Hans, Torben Mikkelsen, Jakob Mann, David Bryce, Adrian Coffey, Michael Harris, David Smith (2004): Site Wind Field Determination using a CW Doppler LIDAR - Comparison with Cup Anemometers at RISØ. Proc. European Wind Energy

Conference, Delft, April 2004, pp. 1-6.

Pierce, Allan D. (1981): Acoustics: An Introduction to Its Physical Principles and Applications, McGraw-Hill Book Company.

Rogers, Anthony L., personal communication, April 2006.

Terray, Eugene A., personal communication, 2006.

Theriault, K.B. (1986): Incoherent multibeam Doppler current profiler performance: Part 1: estimate variance. J. Ocean. Engr., OE-11, pp. 7-15.

Vickers, D. and L. Mahrt (2004); Evaluating formulations of stable boundary layer height. J. Appl. Meteorol. 43, 1736-1749. 


\section{Appendix A}

\section{Beampatterns}

\section{A.1 Beampattern theory}

The Scintec SFAS is a 2 - dimensional phased array antenna consisting of 64 equally spaced sources. In order to compute the beampattern for the SFAS, we must first understand the case of a simple 1-dimensional line array. We want to compute the farfield intensity at an observation point $\mathrm{P}$, which is a vector distance $\mathbf{R}$ from the origin. In order for this calculation to be in the far-field, $\mathbf{R}$ must be much greater than the size of the array. In our array of sources, we denote the $\mathrm{n}^{\text {th }}$ source by $\mathbf{x}_{\mathrm{n}}$. The vector distance from that source to the observation point $\mathrm{P}$ is denoted by $\mathbf{r}_{\mathrm{n}}$, where $\mathbf{r}_{n}=\mathbf{R}-\mathbf{x}_{n}$. Each individual source emits a spherical wave whose amplitude at $\mathrm{P}$ is given by

$$
p_{n}=a_{n} \frac{\exp \left(i k r_{n}\right)}{r_{n}}
$$

where $k_{n}$ is the wavenumber of the radiation and $a_{n}$ is the amplitude. Since the magnitude of $\mathbf{R}$ is much greater than the size of the array $r_{n}$ can be approximated as

$$
r_{n}=\left|\mathbf{R}-\mathbf{x}_{n}\right|=\left[R^{2}-2 \mathbf{R} \cdot \mathbf{x}_{n}+x_{n}^{2}\right]^{1 / 2}=R-x_{n} \sin \vartheta
$$

so that

$$
p_{n} \cong a_{n} \frac{\exp (i k R)}{R} \exp \left(-i k_{\perp} x_{n}\right)
$$

where terms of order $\frac{x_{n}}{R}$ are neglected, and $k_{\perp}$ represents the transverse component of the wavenumber $\mathrm{k}$. The net intensity at $\mathrm{P}$ is therefore given by

$$
I=|p|^{2}=\left[\frac{A}{R}\right]^{2}\left|\sum_{n=0}^{N} w_{n} \exp \left(-i k_{\perp} x_{n}\right)\right|^{2}=\left[\frac{A}{R}\right]^{2} B(\vartheta)
$$


The first factor in (A.4) represents the fall-off in intensity with distance due to spherical spreading. The second factor

$$
B(\vartheta)=\left|\sum_{n=0}^{N_{s}} w_{n} \exp \left(-i k_{\perp} x_{n}\right)\right|^{2}
$$

represents the far-field beampattern of intensity, where the weights $\left\{w_{n}\right\}$ allow more control over sidelobe intensities. Here, $N_{s}$ represents the number of transducers in the array. It is important to note that $\left\{w_{n}\right\}$ must sum to 1 so that $\mathrm{B}(0)=1$. Therefore, the far-field beampattern is determined by the weighted superposition of plane waves from each source.

For uniform weighting, $\omega_{n}=1 / N_{s}$, the sum in A.5 can be computed analytically. We compute this sum for an odd and even number of transducers. For an odd number of transducers, the result is

$$
B(\vartheta)=\left[\frac{\sin \left(k_{\perp} N \Delta x / 2\right)}{\sin \left(k_{\perp} \Delta x / 2\right)}\right]^{2}
$$

and for an even number of transducers the result is

$$
B(\vartheta)=\left[2 \cos \left((N+2) k_{\perp} \Delta x / 4\right) \frac{\sin \left(N k_{\perp} \Delta x / 4\right)}{\sin \left(k_{\perp} \Delta x / 2\right)}\right]^{2}
$$

where $k_{\perp}=k \sin \vartheta$. We used the expressions in A.6 and A.7 to check our numerical results (E. Terray, personal communications).

The 2-dimensional case is computed similarly except the sources now form a grid with locations $x_{n, m}=\left(x_{n}, y_{m}\right)$ and the observation point in the far-field, $\mathrm{P}$, is defined in terms of polar and azimuthal angles as 


$$
\mathbf{R}=R(\cos \varphi \sin \vartheta, \sin \varphi \sin \vartheta, \cos \vartheta)
$$

So that (A.2) becomes

$$
r_{n, m} \cong R-x_{n} \cos \varphi \sin \vartheta-y_{m} \sin \varphi \sin \vartheta
$$

and the field at $\mathrm{P}$ is now

$$
p_{n, m} \approx A w_{n} w_{m} \frac{\exp (i k R)}{R} e d p\left(-i k_{\perp} x_{n} \cos \varphi\right) \exp \left(-i k_{\perp} y_{m} \sin \varphi\right)
$$

where $k_{\perp}=k \sin \vartheta$.

The intensity at $\mathrm{P}$ from an $N_{x} \times N_{y}$ array is

$$
\begin{gathered}
I=|p|^{2}=\sum_{n=1}^{N_{x}} \sum_{m=1}^{N_{y}}\left|p_{n, m}\right|^{2}=\left[\frac{A}{R}\right]^{2} B(\vartheta, \varphi) \\
=\left[\frac{A}{R}\right]^{2}\left|\sum_{n=1}^{N_{x}} \sum_{m=1}^{N_{y}} w_{n} w_{m} \exp \left(-i k_{\perp} x_{n} \cos \varphi\right) \exp \left(-i k_{\perp} y_{m} \sin \varphi\right)\right|^{2}
\end{gathered}
$$

Here, $\left(k_{x}, k_{y}\right)=k_{\perp}(\cos \varphi, \sin \varphi)$, so $B(\vartheta, \varphi)$ can be written in terms of the 1-dimensional amplitude beam pattern as

$$
B(\vartheta, \varphi)=\left|b\left(k_{x} \Delta x\right) b\left(k_{y} \Delta y\right)\right|^{2}
$$


where

$$
\begin{aligned}
& b\left(k_{x} \Delta x\right)=\sum_{n=1}^{N_{x}} w_{n} \exp \left(-i k_{x} x_{n}\right) \\
& b\left(k_{y} \Delta y\right)=\sum_{n=1}^{N_{y}} w_{n} \exp \left(-i k_{y} y_{n}\right)
\end{aligned}
$$

It is convenient to choose the origin in the center of the array, so that for $\mathrm{N}$ even

$$
n=-N / 2, \ldots,-1,1, \ldots, N / 2
$$

and for $\mathrm{N}$ odd

$$
n=-(N-1) / 2, \ldots,-1,0,1, \ldots(N-1) / 2
$$

where $x_{n}=n \Delta x$, and $y_{m}=m \Delta y$.

\section{A.2 Scintec Beampatterns}

For the Scintec 1-D and 2-D vertical beampatterns we use equations A.12 and A.13, respectively. We take $\mathrm{N}=8$ and $\Delta x, \Delta y=0.5 \mathrm{~cm}$ and we multiply by the appropriate weights for Scintec's shading pattern.

The Scintec steered beampatterns $\left( \pm 19^{\circ}, \pm 24^{\circ}\right)$ are computed slightly differently than the vertical beampattern. The 1-D steered beampattern for the unshaded case where $\vartheta_{0}$ is the angle of the steered beam is computed using

$$
b(\vartheta)=\left|\sum_{n=0}^{N_{x}} S_{n} \exp \left(-i k_{x} x_{n} \sin (\vartheta)\right)\right|^{2}
$$


where

$$
S_{n}=\frac{1}{N_{x}} \exp \left(i k_{x} x_{n} \sin \left(\vartheta_{0}\right)\right.
$$

(Clay and Medwin, 1977). Beampattern results for these calculations are shown in Figure 2.4 . 


\section{Appendix B}

\section{ASIS/Sodar Experiment}

\section{B.1 Description of experiment}

The goal of this experiment was to explore the use of sodar deployed on an ASIS buoy to measure wind profiles in the MABL. The work was funded by the Massachusetts Technology Collaborative in connection with an offshore wind power initiative, and had the long-term goal of enabling inexpensive site assessment and monitoring of wind resources without the need of a meteorological tower.

\section{B.2 Instrumentation and Data Acquisition system}

The ASIS buoy used in this experiment was equipped with a variety of instrumentation. A Scintec SFAS sodar was situated in the center of ASIS, on top of a 24-inch aluminum table. The sodar was equipped with a baffle that opened at an angle of $\sim 24$ degrees and extended an additional two feet high. It is important that the sodar was the highest object on the buoy so that other fixed objects were not visible in its beampattern. The sodar was connected to the sodar processing unit (SPU), which was situated below the sodar's table, and was housed in a watertight enclosure box. In order to measure buoy motion, ASIS was outfitted with a BEI Systron Donner IMU consisting of three orthogonal rate gyros and accelerometers. A 3-axis PNI compass was used to measure buoy heading. The IMU and compass were located in the same watertight enclosure box that housed the buoy's Data Acquisition System (DAS).

The ASIS DAS consisted of two PC-104 based stacks that recorded both analog and serial data channels. One stack used a Lippert "Cool Roadrunner II" $333 \mathrm{MHz}$ Pentium PC-104 card that was responsible for recording data from all sensors except for the sodar. Data was recorded on an $80 \mathrm{~GB}$ hard drive. This stack also had a Diamond systems MM32 analog-to-digital converter card, a ConnectTech Extreme 8-port RS-232 serial card, a Parvus 4-port 10/100 Mbit Ethernet switch, and a Tri-M IR-104 relay card. The Ethernet switch created a local area network within the DAS, and the relay card provided a way to switch power on and off to each sensor. A second stack was necessary to log the data 
from the sodar. This stack consisted of a Lippert "Cool Roadrunner III" $650 \mathrm{MHz}$ Pentium PC-104 card, and was connected to the LAN via the Parvus Ethernet switch. Both stacks, along with the compass and IMU were housed in a Rose Bopla watertight enclosure that sat on the top deck of ASIS. To enable remote access, the DAS was connected to a Freewave spread spectrum $900 \mathrm{MHz}$ modem, which was housed in a Rose Bopla watertight enclosure. The freewave radio was affixed to one of the legs of the sodar table. A picture of the DAS, IMU, and compass is shown in Figure B.1.

In order to track the buoy's location, we included both ARGOS and GPS receivers. The ARGOS was an independent system that ran on its own batteries. Both the ARGOS receiver and batteries are situated in a watertight tube that was attached to one of the legs of the sodar table. The Garmin GPS receiver was attached to another of the table's legs. In accordance with Coast Guard regulations, ASIS was also equipped with a marine light and a radar reflector. 


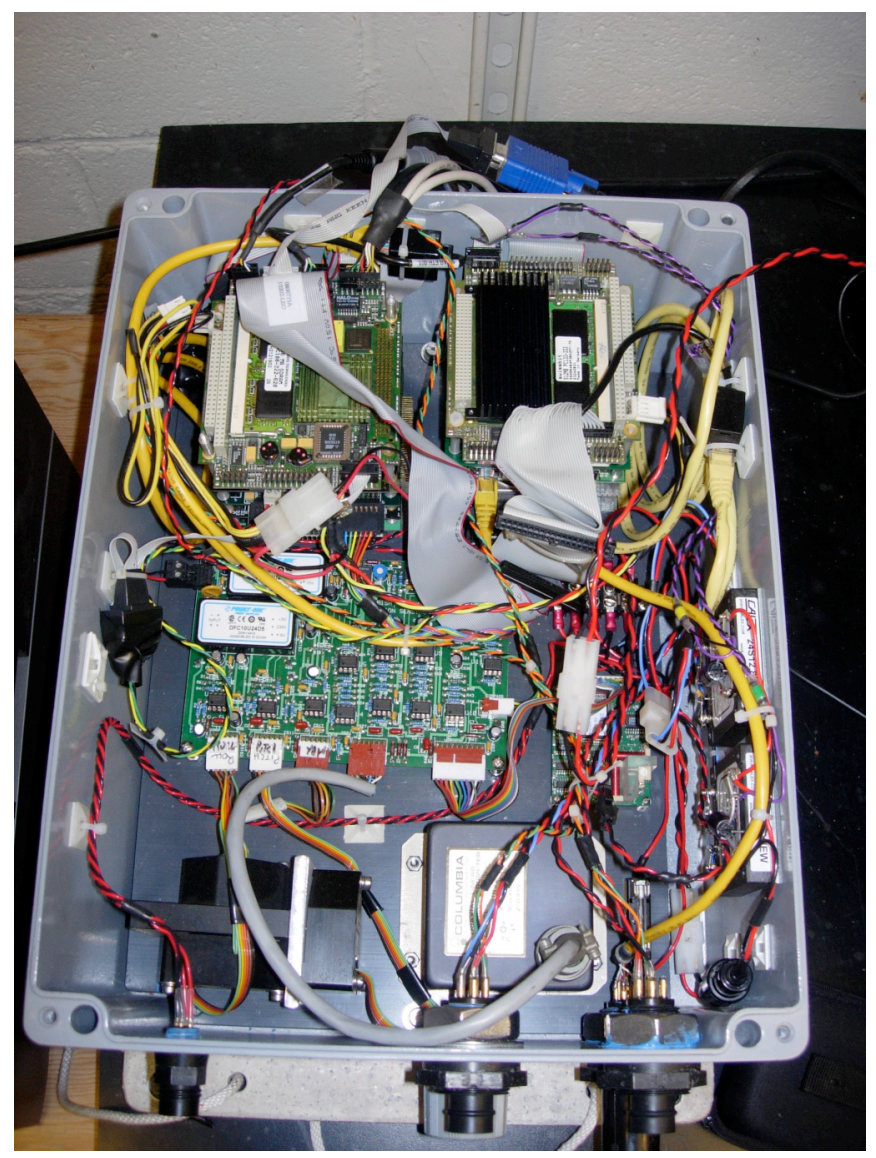

Figure B.1 DAS and IMU enclosure.

A layout of the instrumentation aboard ASIS is shown in Figure B.2. The Scintec SFAS sodar is situated in the center of the pentagonal deck atop an aluminum table. The sodar's baffle is seated on top of the instrument and is supported by aluminum arms that are welded to the sodar's table. The SPU is visible below the aluminum table. The DAS is the grey box situated in front of the SPU. The marine light, which is affixed to a post, is visible in the upper-left corner and the GPS (small white antenna) is visible in the upper-right corner. The radar reflector is the larger white cylindrical enclosure that is partially obscured by the aluminum table. The ARGOS is located to the right of the DAS. 


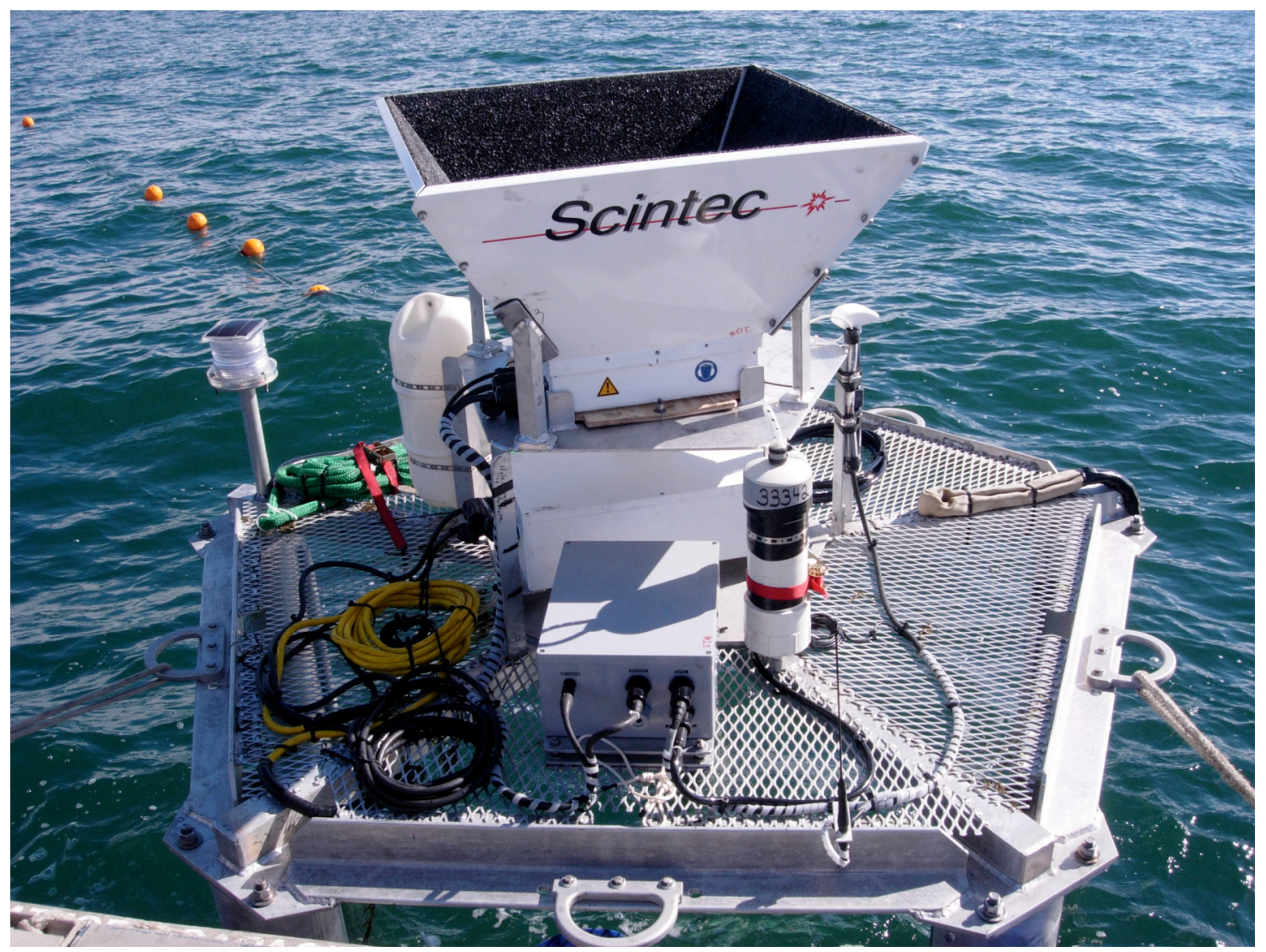

Figure B.2 ASIS deck layout.

\section{B.2 Power}

ASIS is equipped with five battery cans located at its foot. Each can houses 490 Alkaline D-cell batteries that provide $12 \mathrm{KWh}$ of energy per can. This amounts to $60 \mathrm{KWh}$ of energy for the ASIS buoy. Batteries are available pre-assembled from Matthews Assoc. at $\sim \$ 800$ per can. The power budget for our experiment is about $125 \mathrm{~W}$ (see Table B.1). With 60KWh available, our instrumentation can be powered continuously for 20 days, or for longer at a lower duty cycle.

It is important to note that the sodar and its computer are responsible for about $67 \%$ of the power required for this experiment. This is in part due to the fact that the sodar requires a $650 \mathrm{MHZ}$ Pentium processor to collect data. Software modifications could potentially enable the Scintec SFAS sodar to log data in a close to raw format, which would cut down on processor speed and save about 8 Watts of power. 


\section{Power Budget}

\begin{tabular}{|l|l|l|c|}
\hline \multicolumn{1}{|c|}{ Item } & \multicolumn{1}{|c|}{ Manufacturer } & \multicolumn{1}{c|}{ Model } & Power Req'd (W) \\
\hline $333 \mathrm{MHz}$ Pentium CPU & Lippert & Cool Road Runner II & 6.0 \\
\hline $650 \mathrm{MHz}$ Pentium CPU & Lippert & Cool Road Runner III & 15.5 \\
\hline 5 Port Ethernet Switch & Parvus & Switch 104 & 1.5 \\
\hline 20 Output Relay Card & Tri-M Systems & IR 104 & 0.2 \\
\hline 32 Channel 16 bit A/D & Diamond Systems & Diamond-MM-32-AT & 2.0 \\
\hline PC 104 Power Supply & Tri-M Systems & HE 104 & 0.5 \\
\hline 8 Port Rs-232 Serial Card & Connect Tech & Xtreme/104 RS-232 & 1.0 \\
\hline Wireless comms & Freewave & Ethernet radio & 0.3 \\
\hline & & \multicolumn{1}{|c|}{ DAS Subtotal } & 27.0 \\
\hline Angular Rate Sensors (3) & BEI Systron Donner & QRS11 & 3.0 \\
\hline Compass & Precision Navigation Inc. & TCM-2 & 1.0 \\
\hline Linear Accelerometers & Columbia Research Labs & SA-307TX & 0.6 \\
\hline SFAS & Scintec & SFAS & 93 \\
\hline & & \multicolumn{1}{|c|}{ TOTAL } & 124.6 \\
\hline
\end{tabular}

Table B.1 Fully instrumented ASIS power budget.

\section{B.3 Communications}

Communication with the ASIS buoy was enabled through the use of two $900 \mathrm{MHz}$ Freewave Spread Spectrum Ethernet Modems. One Freewave was located on ASIS and was connected to the DAS via an Ethernet connection. A second Freewave was located on the MVCO ASIT tower and was connected to a top-side node. An electro-optic power cable established the communication of ASIT with the shore. The VNC (Virtual Network Computing) software package was used to view and interact with the computers on board ASIS remotely. This allowed for full control of all instrumentation on the buoy from a shore-based computer. Should the Freewaves fail, communication can be established with the computers on ASIS from a ship via a long Ethernet cable that plugs into the DAS.

\section{B.4 ASIS towing and Mooring}

Since a fully instrumented ASIS weighs about $2900 \mathrm{lbs}$ and is $10 \mathrm{~m}$ long, it typically is deployed using a large-scale oceanographic vessel such as the R/V Oceanus or Knorr. However, the cost of the use of one of these vessels ( $>\$ 19,000 /$ day) was not possible 
within our budget, so we had to develop a way to tow, deploy and recover ASIS using a smaller more affordable vessel like the R/V Tioga, which has a day-rate of $\$ 2400$. A picture of the Tioga is shown in Figure B.3 and its specifications are listed in Table B.2.

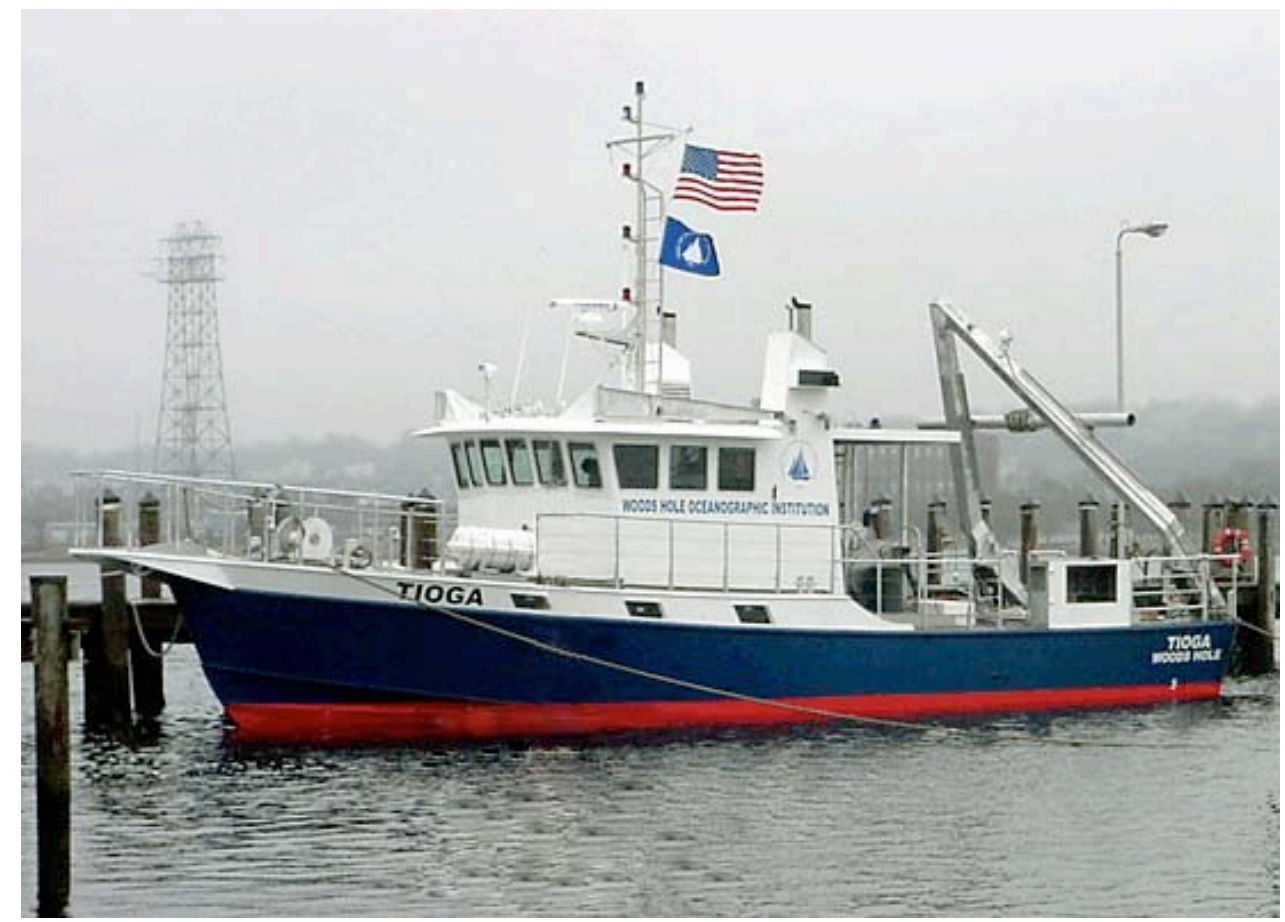

Figure B.3 WHOI's coastal research vessel R/V Tioga.

\begin{tabular}{|c|c|c|c|}
\hline \multicolumn{4}{|c|}{ R/V Tioga Specifications } \\
\hline Dimensions & Length $60 \mathrm{ft}$ & Draft $5 \mathrm{ft}$ & Beam $17 \mathrm{ft}$ \\
\hline Space & Deck $300 \mathrm{ft}^{2}$ & Lab $200 \mathrm{ft}^{2}$ & \\
\hline Speed & Max - 22.5 kts & Cruising $-18.0 \mathrm{kts}$ & Range $900 \mathrm{~nm}$ \\
\hline Equipment & $\begin{array}{l}\text { Trawl winch ( } 800 \mathrm{~m} \text { of } \\
5 / 16 \text { " } 3 \times 19 \text { wire) }\end{array}$ & $\begin{array}{l}\text { CTD winch }(750 \mathrm{~m} \\
0.322 \text { " 3-cond } \\
\text { cable) }\end{array}$ & $\begin{array}{l}\text { A-frame } \\
\text { Crossbeam: } 4600 \mathrm{lbs} \\
\text { Peak: } 2000 \mathrm{lbs}\end{array}$ \\
\hline
\end{tabular}

Table B.2 R/V Tioga's Specifications. 
Since the weight of the buoy exceeds the maximum weight that the TIOGA's A-frame can handle, it must be towed. We have successfully towed ASIS in relatively calm conditions at 9 knots using a line attached to the buoy's foot. This towing line is passed through a sheave on the TIOGA to its trawl winch. The line is stopped off to the deck of the TIOGA to relieve the winch throughout the tow. The foot and batteries of ASIS are the only things that rest above water, so all other instrumentation must be deployed on station. A picture of ASIS being towed is shown in Figure B.4. Once ASIS has been deployed, the towing line is attached to the main deck to facilitate recovery.

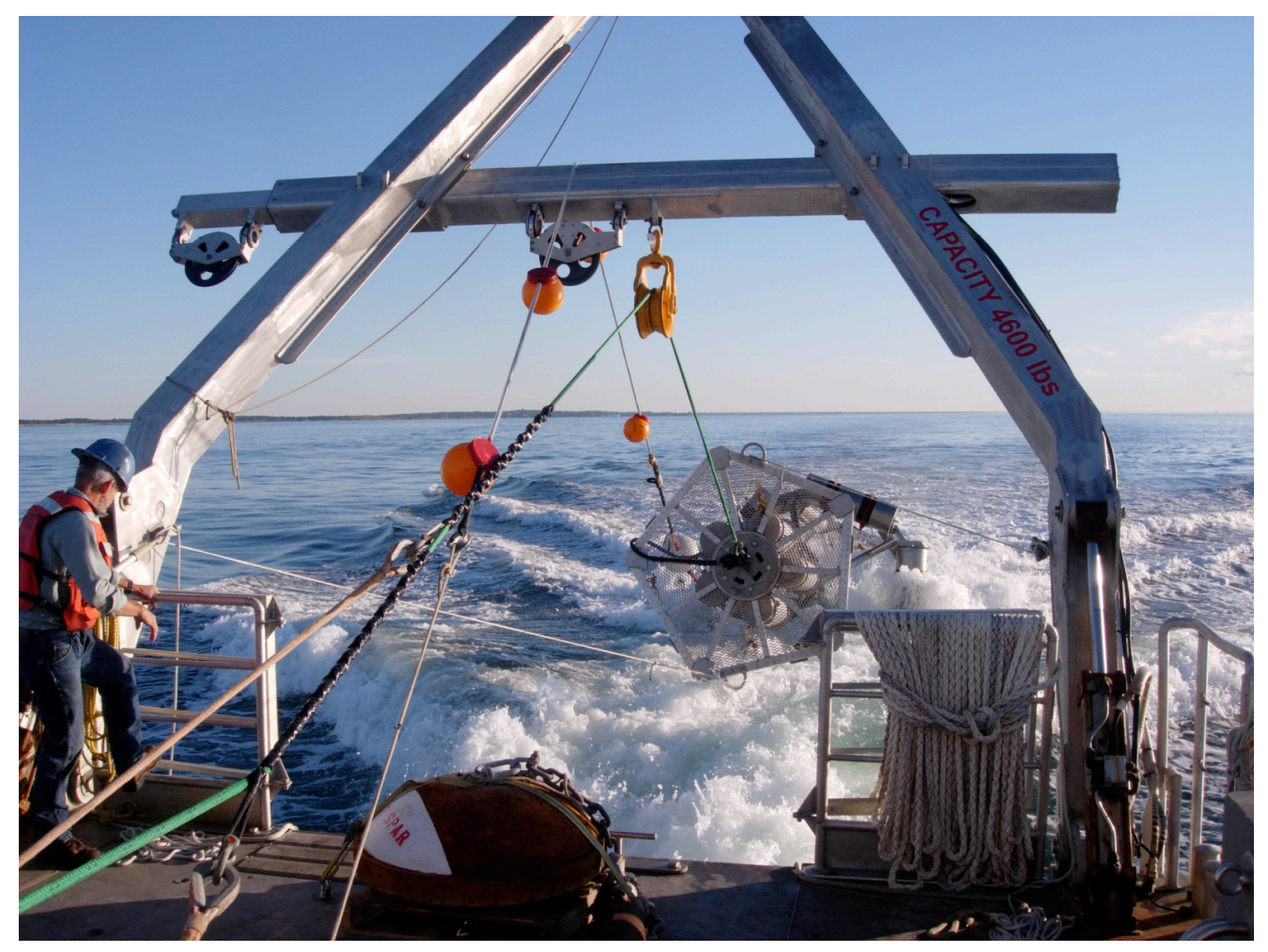

Figure B.4 ASIS being towed at 9 knots.

Since ASIS has little reserve buoyancy, it must be tethered to a surface mooring nearby. The tether system used in this experiment consists of $50 \mathrm{~m}$ of $1 / 2$ inch spectra lined with 14 and 6 inch orange floats. The tether is attached to a spherical surface mooring. Since the surface mooring is a potential navigational hazard, it too is equipped with a marine radar reflector and light. A picture of our fully instrumented ASIS tethered to its surface mooring is shown in Figure B.5. 


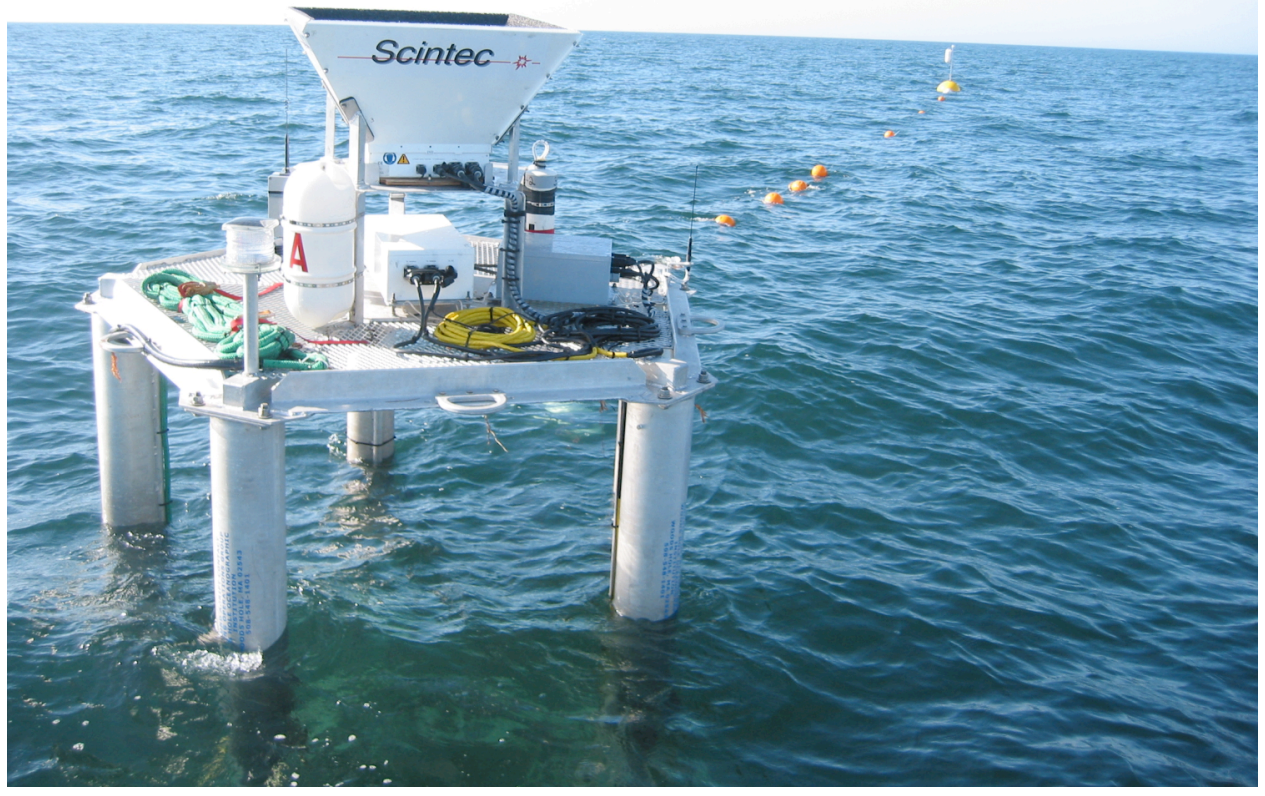

Figure B.5 A fully instrumented ASIS tethered to its surface mooring. 


\section{Appendix C}

\section{ASIS Tilting Model}

We developed a model to predict the static tilt of the ASIS buoy under different conditions of steady winds and currents. First, ASIS was decomposed into several components whose cross sectional area and distance to the tether point are determined. A "torque coefficient", $D$, for the buoy in air and water is then computed using the formula

$$
D=\frac{1}{2} \rho \sum_{n} C_{d_{n}} r_{n} A_{n}
$$

where $\rho$ is the density of the medium (air or water), $C_{d_{n}}$ is the drag coefficient, $r_{n}$ is the distance from the $n^{\text {th }}$ structural element of ASIS to the tether point of the buoy, and $A_{n}$ is the cross-sectional area of the $n^{\text {th }}$ component as seen by the air or water. The torque on the buoy in both air and water is computed from

$$
\mathbf{T}=D^{*}|U| * \mathbf{U}
$$

where $\mathbf{T}$ is the vector torque of the buoy in air or water, $D$ is the "torque coefficient" in air or water, and $\mathrm{U}$ is the velocity of the current or wind. The torques due to the air and water-side flows are summed and set equal to the gravitational torque. Tilts are computed via

$$
\theta=T /(M g * d r)
$$

where $\theta$ is the tilt angle about the axis of torque, $T$ is the net torque due to winds and currents, $M$ is the mass of ASIS, $g$ is the gravitational acceleration, and $d r$ is the distance between the center of buoyancy and the center of mass of the buoy (CB-CG). Verification of this model is discussed in the main text of the thesis (see Figure 4.16). 


\section{Appendix D \\ Sensor Calibrations}

The accelerometers, rate gyros and compass were calibrated in the laboratory using a mechanically driven tilting table (Appendix F). Each instrument was calibrated with respect to its local axes, which in turn are related to a set of axes relative to the box housing the data acquisition system (DAS). The sensors were calibrated as assembled in the box. A sketch of the relationship between the various coordinate systems is shown in Figure D.1. Note that the compass nominally has two coordinate systems: one for the magnetometers and one for the tilt sensors. The magnetometer coordinates are labeled $\mathrm{X}$, $\mathrm{Y}$, and Z, while the tilt sensor coordinates are labeled B and C. Compass axes B and C are nominally oriented in the same direction as axes $\mathrm{X}$ and $\mathrm{Y}$, respectively.

It is important to note that the DAS instrumentation was calibrated as an entire system. Data flow from the sensors first passed through a signal conditioning board where it was low-pass filtered ( $3 \mathrm{~dB}$ roll-off at $5 \mathrm{~Hz}$ ), and then digitized with a Diamond systems MM3216 bit A-D converter. So the measured calibration coefficients convert from the A-D output (in bits) to the relevant physical units. 


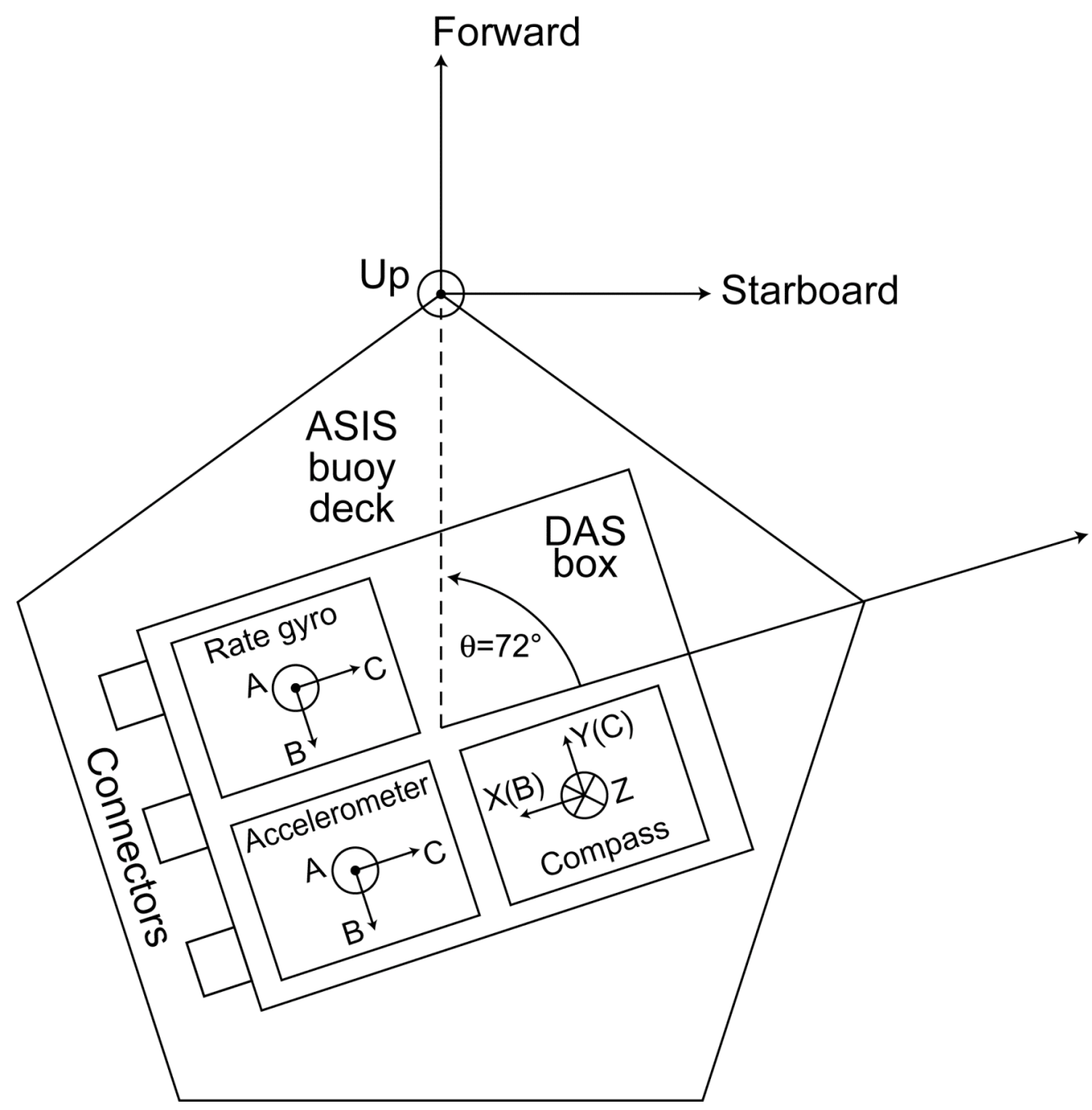

Figure D.6 A sketch of the orientations of the axes inside the DAS.

\section{Accelerometers:}

Acceleration was measured using a Columbia Research Laboratory SA-307 HPTV triaxial accelerometer. The accelerometer was calibrated by a series of static tilts. Accelerometer axes B and C were calibrated in the same manner, whereas axis A was calibrated in a slightly different fashion. To calibrate the $\mathrm{B}$ and $\mathrm{C}$ axes of the accelerometers, the box was statically tilted around each axis. The tilts were measured by a digital protractor (with a resolution of $0.1^{\circ}$ ) and ranged from about -20 to 20 degrees. The accelerometer output from the digitizer (A/D) was then averaged over the duration of each tilt in order to reduce noise. The average was fit to the sine of the angle of tilt using a linear regression. The fits for the B and C axes are shown in Figures D.2 and D.3. 


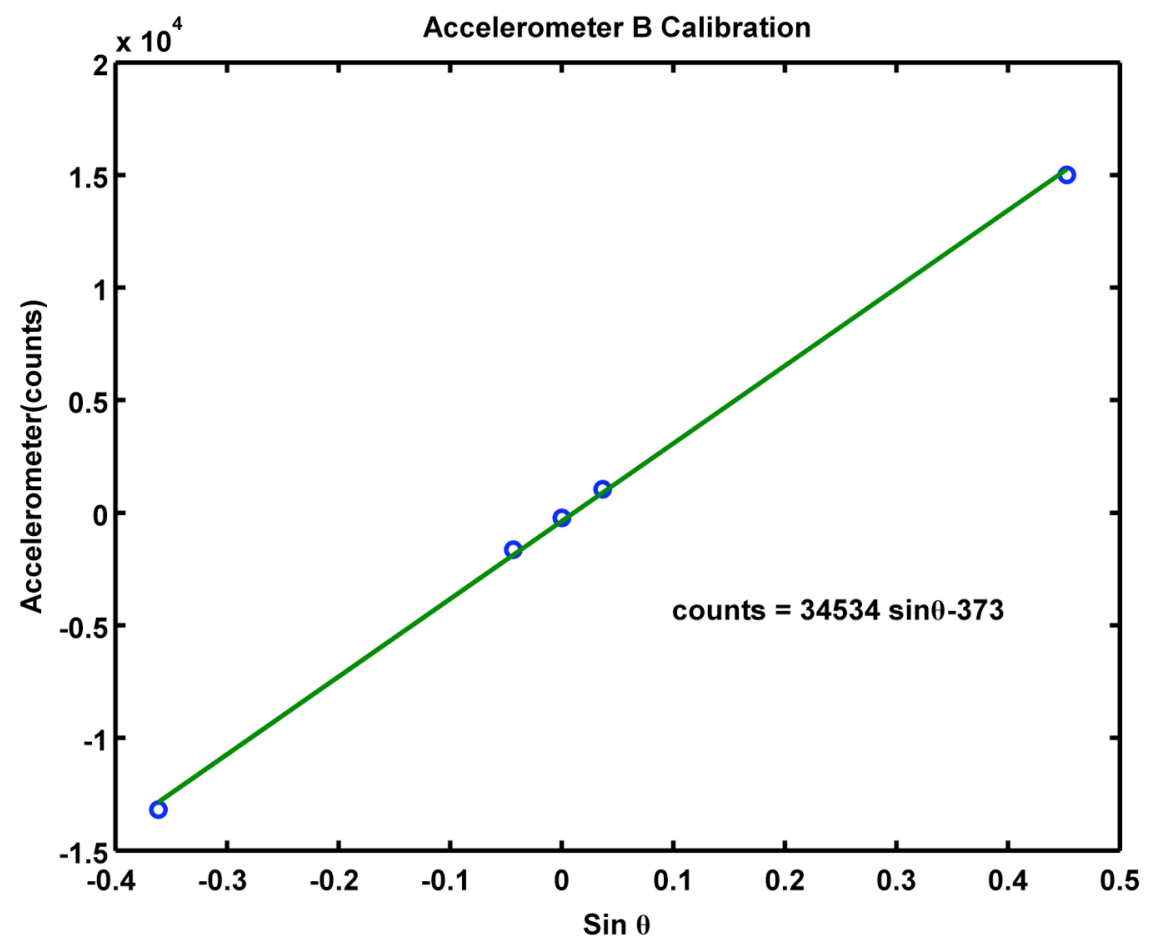

Figure D.2 Accelerometer axis B calibration results plotted with a linear fit.

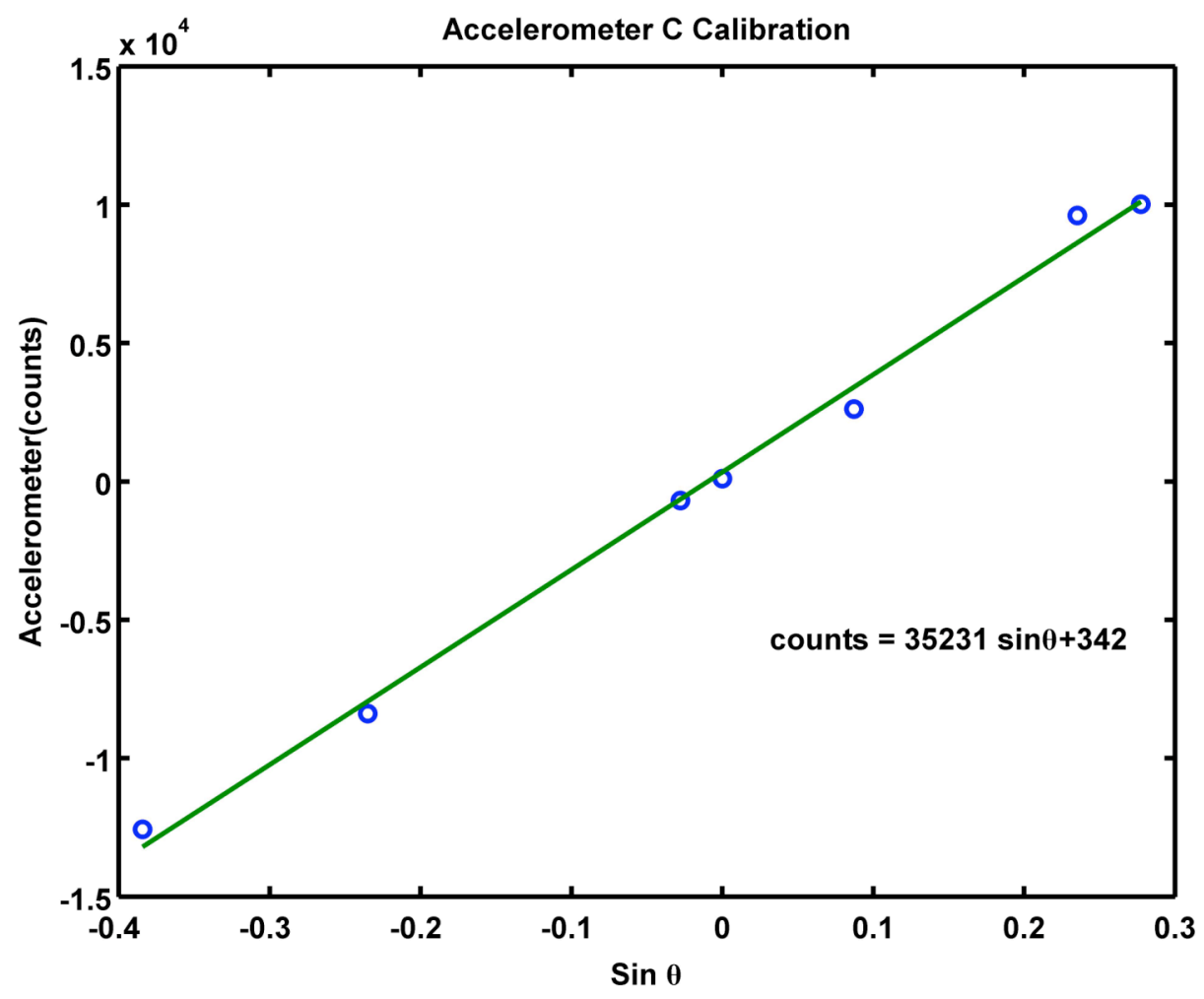

Figure D.3 Accelerometer axis C calibration results plotted with a linear fit. 
We used a slightly different procedure for calibrating accelerometer axis A. In this case, the DAS was statically rotated around axis C for angles ranging from -90 to 90 degrees. Tilts again were measured by a digital protractor. Since the response for this axis is a cosine, it was necessary in this case to rotate the box over larger angles so that tilts in axis A would be large enough to be seen. As before, the accelerometer output was averaged over the duration of the tilt (which was about four minutes) in order to decrease the standard error of the sample mean. The average was related to the cosine of the tilt angle and was fit with a linear equation. The result of this calibration can be seen in Figure D.4.

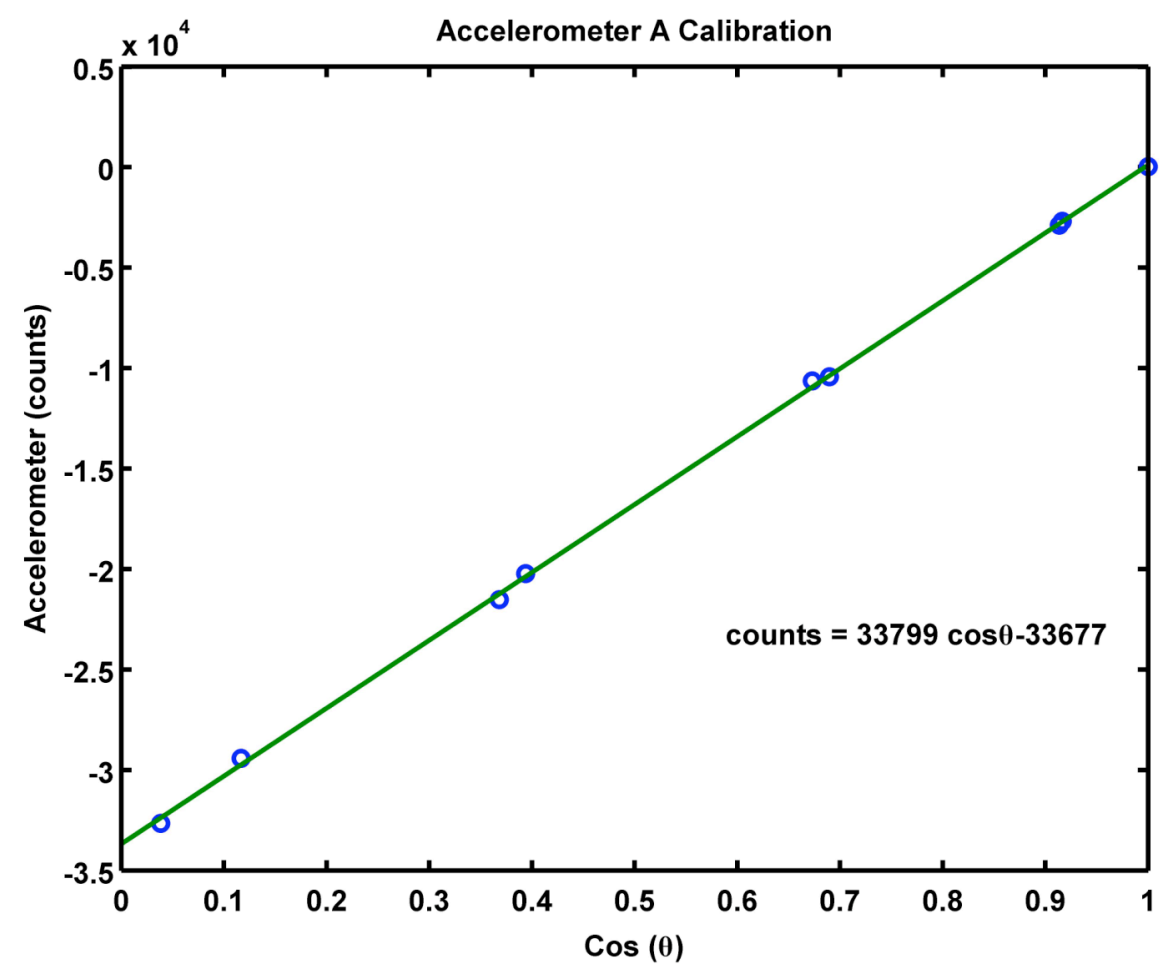

Figure D.4 Accelerometer axis A calibration results plotted with a linear fit.

\section{$\underline{\text { Rate Gyros: }}$}

Angular rates were measured using three orthogonal Systron-Donner QRS-11 solid-state rate gyros. The three rate gyros were calibrated using our mechanically driven tilting table, which is described in Appendix F of this report. In order to investigate the frequency dependence in the rate gyro output, axes A, B and C were calibrated for three 
different periods of rotation: 5 seconds, 8 seconds, and 20 seconds. Instantaneous platform tilt was measured by a potentiometer (see Appendix F for details) attached to the shaft of the tilting table. The rate gyros were digitized at $20 \mathrm{~Hz}$ using the 16-bit A-D in the DAS. We digitized the potentiometer using a separate 10-bit A-D that was asynchronous with the DAS A-D. Hence we could not directly compare the corresponding time series, and had instead to make a statistical comparison. A typical rate gyro spectrum used in this calibration is shown in Figure D.5.

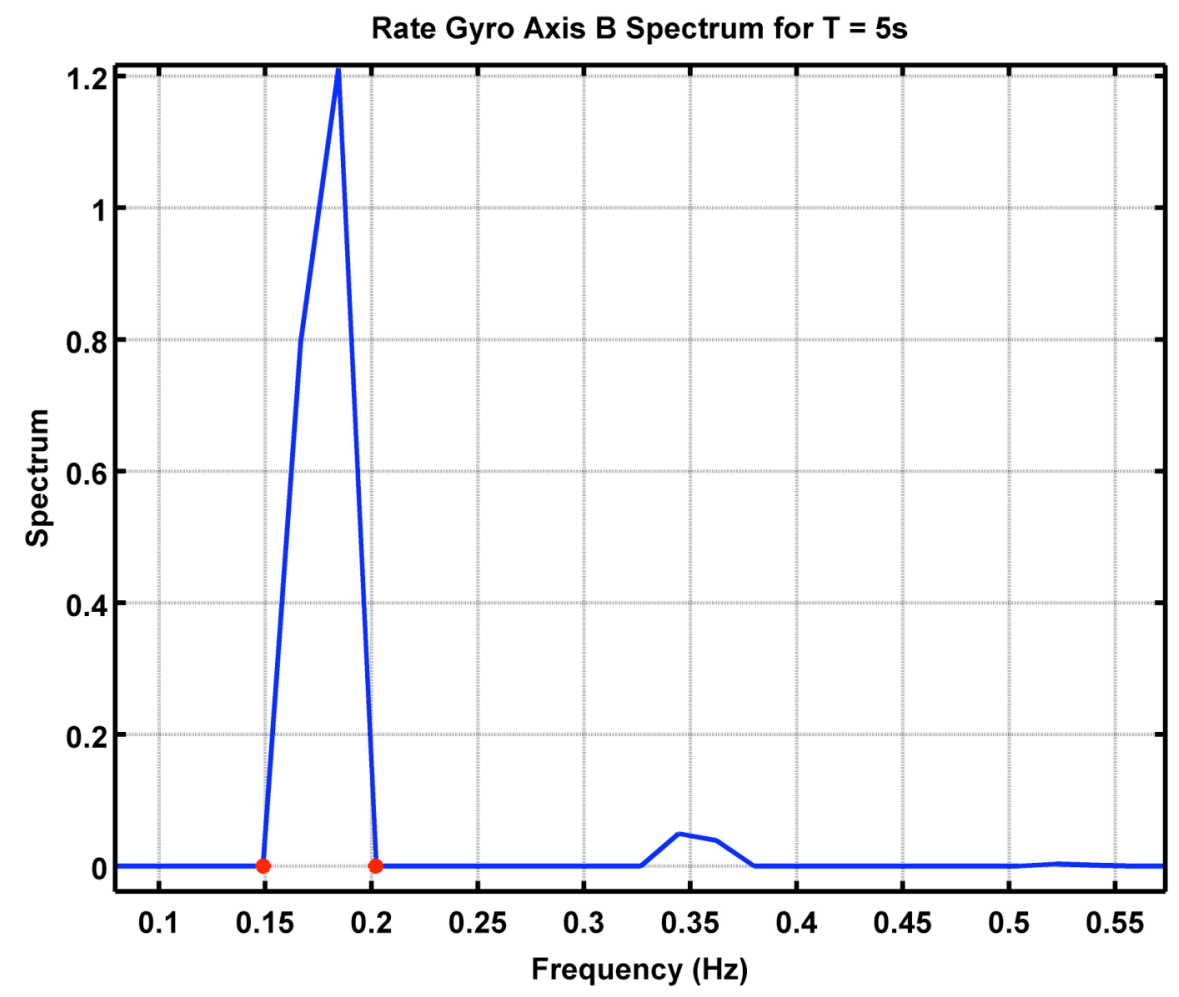

Figure D.5 Rate Gyro Axis B spectrum for $\mathbf{T}=\mathbf{5 s}$.

The conversion from $\mathrm{A} / \mathrm{D}$ counts to $\mathrm{rad} / \mathrm{s}$ was computed as

$$
\alpha=\sqrt{\frac{\int S_{\theta} \omega^{2}}{\int S_{\Omega}}}\left(\frac{\mathrm{rad}}{\mathrm{s}} / \text { counts }\right)
$$


where $S_{\Omega}\left((\mathrm{rad} / \mathrm{s})^{2} / \mathrm{Hz}\right)$ is the rate gyro spectrum, $S_{\theta}\left(\mathrm{rad}^{2} / \mathrm{Hz}\right)$ is the potentiometer spectrum, $\omega$ is frequency $(\mathrm{rad} / \mathrm{s})$, and the integration is over the dominant peak in the spectrum from $0.15-0.2 \mathrm{~Hz}$.

We included only the first harmonic in our calibration because digitization noise in the potentiometer spectrum is amplified at high frequencies by the factor of $\omega^{2}$. This is illustrated in Figure D.6, which shows a comparison of the rate gyro and potentiometer rate spectra plotted on a vertical logarithmic axis. We investigated the effect of this noise on the calibration coefficients by comparing the result to that based on the ratio of the standard deviations of the differentiated potentiometer and rate gyro signals (e.g. using the full spectral bandwidth). The results from the latter were inconsistent with errors ranging from $10-200 \%$.

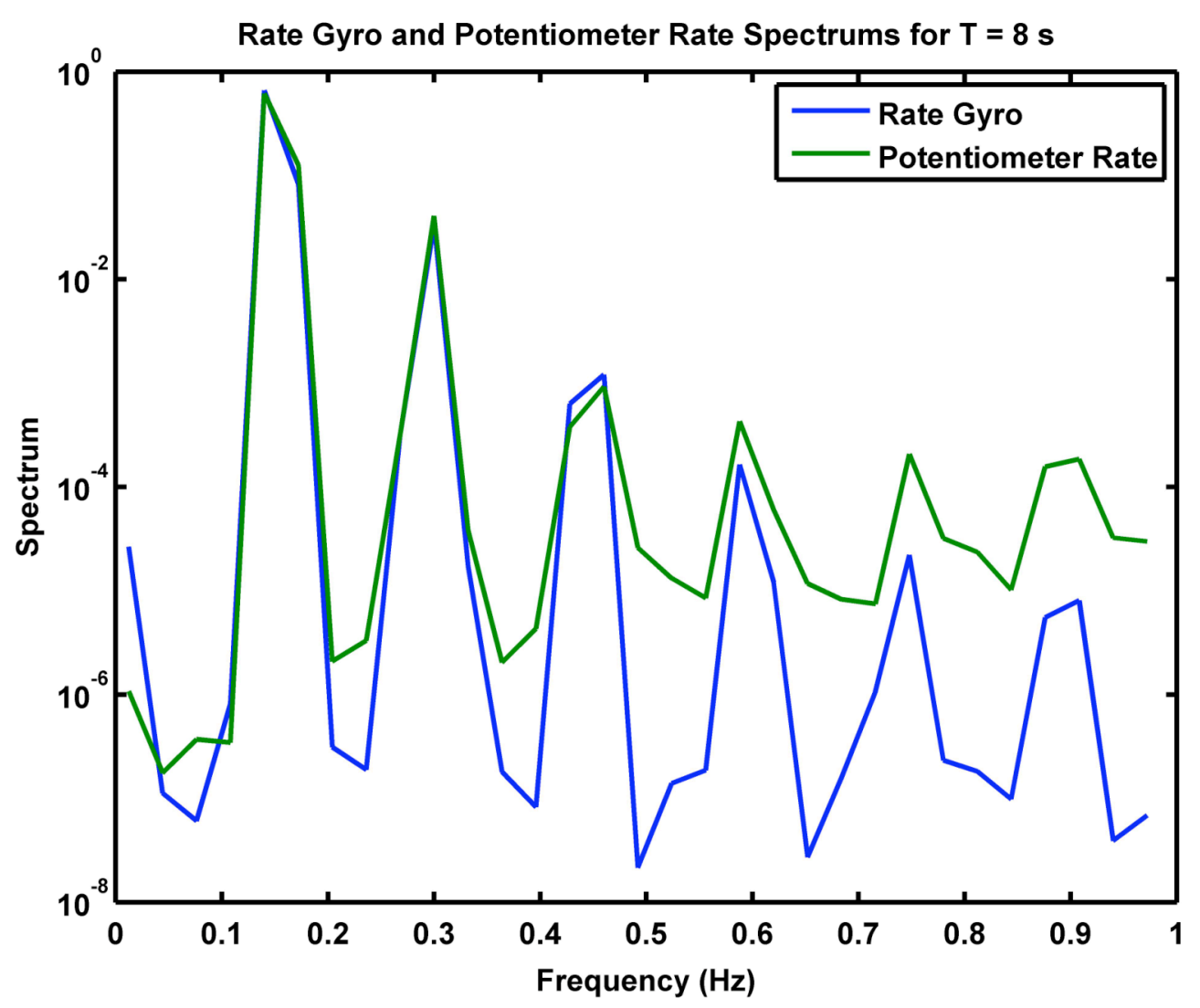

Figure D.6 A comparison of the logarithm of the rate gyro and potentiometer rate spectra vs. frequency. 
Conversions from counts $(C)$ to angular rate $(\mathrm{rad} / \mathrm{s})$ for the rate gyros are given in Table D.1. Calibration factors vary slightly over frequency by $1-2 \%$. The source of this variability is unknown, and we have taken our calibration coefficient as the mean over the three tilting frequencies.

\begin{tabular}{|c|c|c|c|c|}
\hline \multicolumn{7}{|c|}{ Rate Gyro Calibration Summary } \\
\hline Axis & $\begin{array}{c}\text { 5 Second } \\
\text { Period }\end{array}$ & $\begin{array}{c}\text { 8 Second } \\
\text { Period }\end{array}$ & $\begin{array}{c}\text { 20 Second } \\
\text { Period }\end{array}$ & $\begin{array}{c}\text { Mean for all } \\
\text { Periods }\end{array}$ \\
\hline Axis A & $\Omega=2.581 \times 10^{-5} C$ & $\Omega=2.505 \times 10^{-5} C$ & $\Omega=2.581 \times 10^{-5} C$ & $\Omega=2.556 \times 10^{-5} C$ \\
\hline Axis B & $\Omega=1.547 \times 10^{-5} C$ & $\Omega=1.533 \times 10^{-5} C$ & $\Omega=1.586 \times 10^{-5} C$ & $\Omega=1.554 \times 10^{-5} C$ \\
\hline Axis C & $\Omega=1.598 \times 10^{-5} C$ & $\Omega=1.559 \times 10^{-5} C$ & $\Omega=1.509 \times 10^{-5} C$ & $\Omega=1.555 \times 10^{-5} C$ \\
\hline
\end{tabular}

Table D.2 Rate Gyro calibration summary.

An investigation of the rate gyros at rest was also performed in the laboratory. Data was taken for four minutes while the DAS was sitting flat on the bench. The spectrum of the results for each rate gyro axis is shown in Figure D.7. 

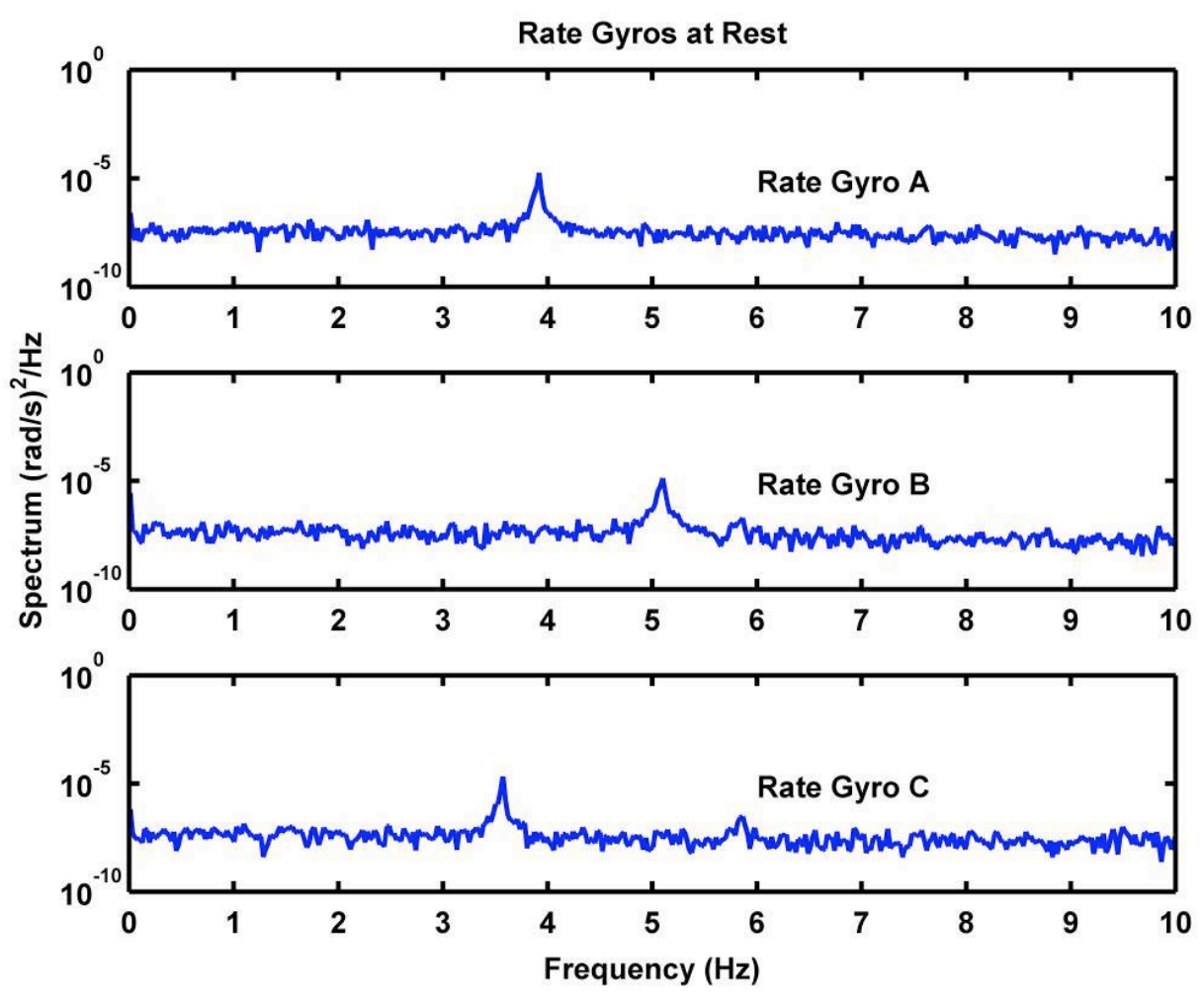

Figure D.7 Spectra of rate gyros at rest.

A summary table of the results of the rate gyro data taken at rest is shown below (Table D.2)

\begin{tabular}{|ll|l|l|l|}
\hline & & \multicolumn{1}{c|}{ Axis A } & \multicolumn{1}{c|}{ Axis B } & \multicolumn{1}{c|}{ Axis C } \\
\hline $\mathbf{N}$ & {$\left[(\mathbf{r a d} / \mathbf{s})^{2} / \mathbf{H z}\right]$} & $1.353 \times 10^{-7}$ & $8.933 \times 10^{-8}$ & $8.316 \times 10^{-8}$ \\
\hline $\mathbf{f}_{\text {res }}$ & {$[\mathbf{H z}]$} & 3.928 & 5.104 & 3.583 \\
\hline$\sigma_{N}$ & {$[\mathbf{r a d} / \mathbf{s}]$} & $1.163 \times 10^{-3}$ & $9.452 \times 10^{-4}$ & $9.119 \times 10^{-4}$ \\
\hline$\sigma_{\text {res }}$ & {$[\mathbf{r a d} / \mathbf{s}]$} & $8.292 \times 10^{-4}$ & $8.581 \times 10^{-4}$ & $8.526 \times 10^{-4}$ \\
\hline
\end{tabular}

Table D.3 A summary of rate gyro results at rest.

where $\mathbf{N}$ is the background noise level of the spectrum, $\mathbf{f}_{r e s}$ is the frequency of the resonant peak, $\sigma_{N}$ is the standard deviation of the noise, and $\sigma_{r e s}$ is the standard deviation of the resonant peak. The peak is resolved by our digitization rate, but lies above the wave band of frequencies, and hence can be filtered. 


\section{Compass:}

Heading was measured using a Precision Navigation Inc. (PNI) TCM-2 Compass. This unit measures three axes of magnetic field and has a 2-axis liquid-level tilt sensor. The tilt sensor axes of the compass were calibrated statically, by tilting the box around each axis for angles in the range of -20 to 20 degrees, where the tilt angles were measured by a digital protractor. The output of the compass (which is in degrees) did not fluctuate much, so data could be recorded by looking at a computer monitor hooked up to the DAS and noting by hand the tilt angle recorded by the compass. The tilt angle measured by the compass was linearly regressed against the tilt measured by the digital protractor.

Results for the calibration of the compass tilt axes B and C are given in Figures D.8 and D.9.

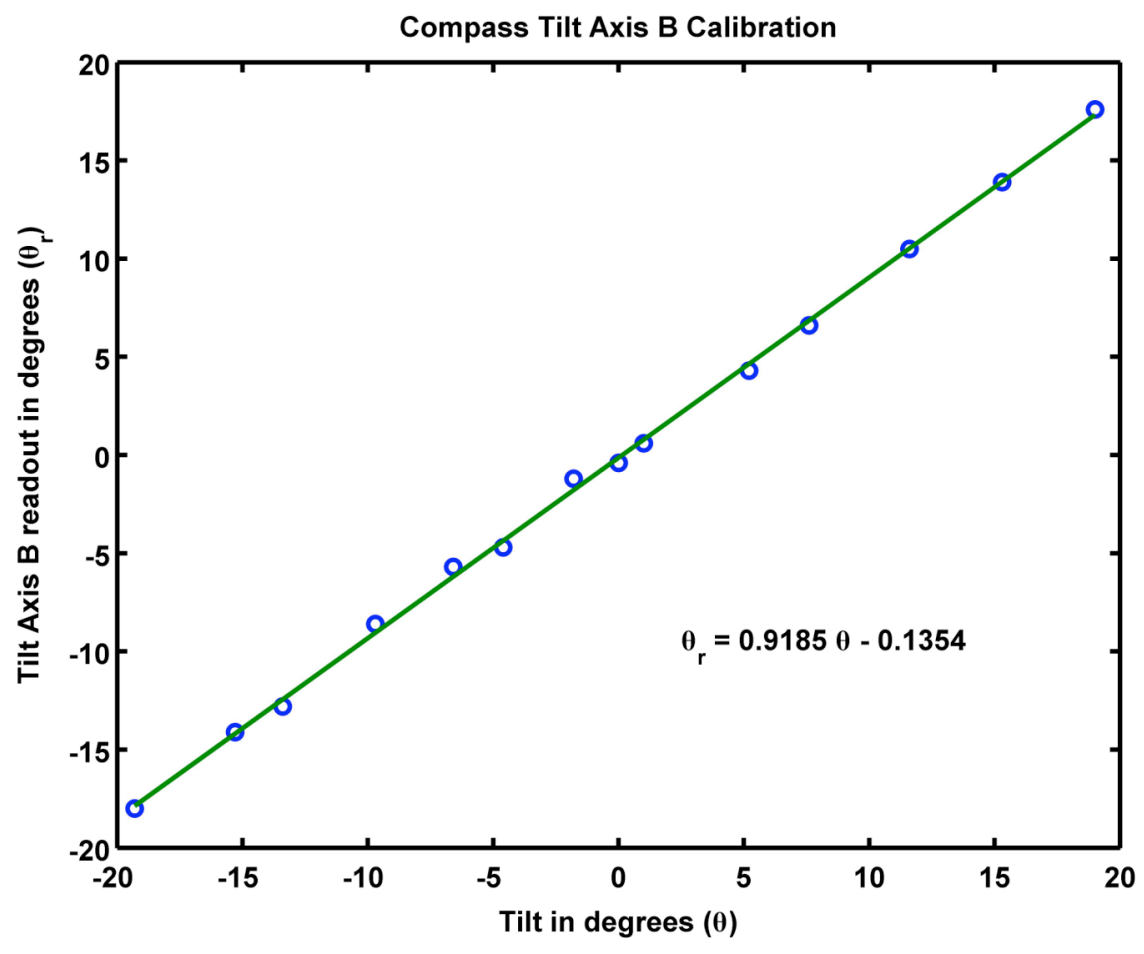

Figure D.8 Compass Tilt Axis B calibration results plotted with a linear fit. 


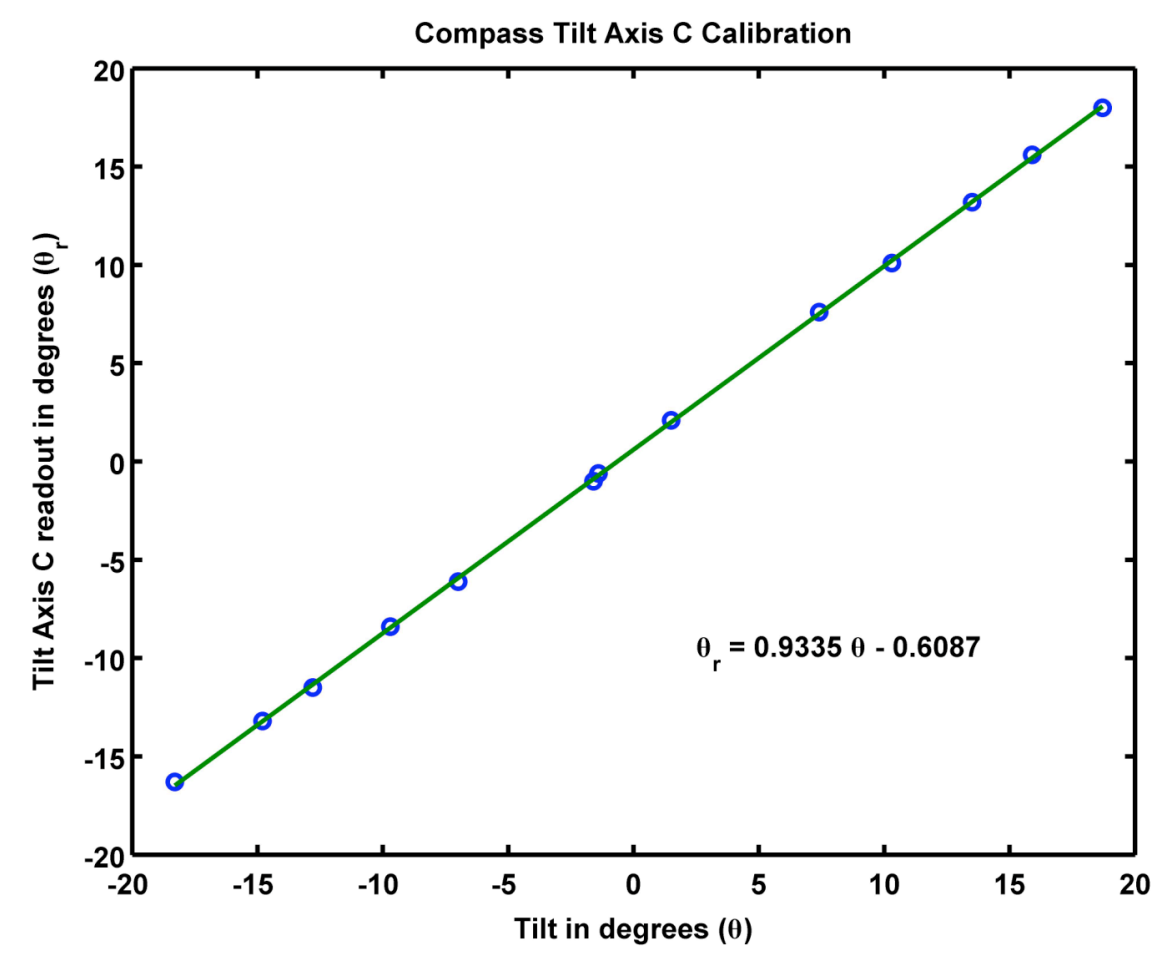

Figure D.9 Compass Tilt Axis C calibration results plotted with a linear fit.

The SODAR draws $\sim 8 \mathrm{~A}$ while running. It is powered through two DC-DC converters located close to the compass, and we were concerned about the possible effect of this high current on the measurement of heading. As a quick check, we first looked at the output of the PNI compass while pointing it roughly North (as measured by a handheld compass) inside the laboratory. The output of the compass was recorded for several minutes with the DC-DC converters both off and on (passing current into a dummy 8A resistive load). The DC-DC converters changed the heading by approximately 10 degrees (Table D.3).

\begin{tabular}{|c|c|}
\hline \multicolumn{2}{|c|}{ Compass North Calibration } \\
\hline State of DC-DCs & Mean Magnetic Output \\
\hline OFF & $17.452^{\circ}$ \\
\hline ON & $7.114^{\circ}$ \\
\hline
\end{tabular}

Table D.3 Compass North Calibration. 
Because of this result, we calibrated the magnetic heading readout of the compass over 360 degrees with the DC-DC converters both off and on (again with a dummy load). We conducted the procedure outside in an area having a fairly uniform magnetic field. The DAS was rotated in increments of 15 degrees on a leveled piece of plywood. Each heading was recorded for several minutes, and the mean of the instrument's heading was compared to the magnetic heading measured by a handheld compass (resolution of $1-2^{\circ}$ ). The calibration was performed with the DC-DCs both on and off. Results in both cases were slightly non-linear, and therefore were fitted with a third-degree polynomial of the form

$$
H=\sum_{n=0}^{3} a_{n} I^{n}
$$

where $a_{n}$ are fitting coefficients, $H$ is the handheld compass heading and $I$ is the instrument heading. The polynomial coefficients for the two cases are given in Table D.4.

\begin{tabular}{|c|c|c|}
\hline Coefficient & DC-DCs ON & DC-DCs OFF \\
\hline$a_{0}$ & -2.9358 & -5.2388 \\
\hline$a_{1}$ & 0.7315 & 0.5249 \\
\hline$a_{2}$ & 0.0035 & 0.0059 \\
\hline$a_{3}$ & $-1.2887 \times 10^{-5}$ & $-2.0336 \times 10^{-5}$ \\
\hline
\end{tabular}

Table D.4 Calibration Coefficients for the PNI compass.

Plots of the calibration results and the polynomial fit are shown in Figures D.10 and D.11. 


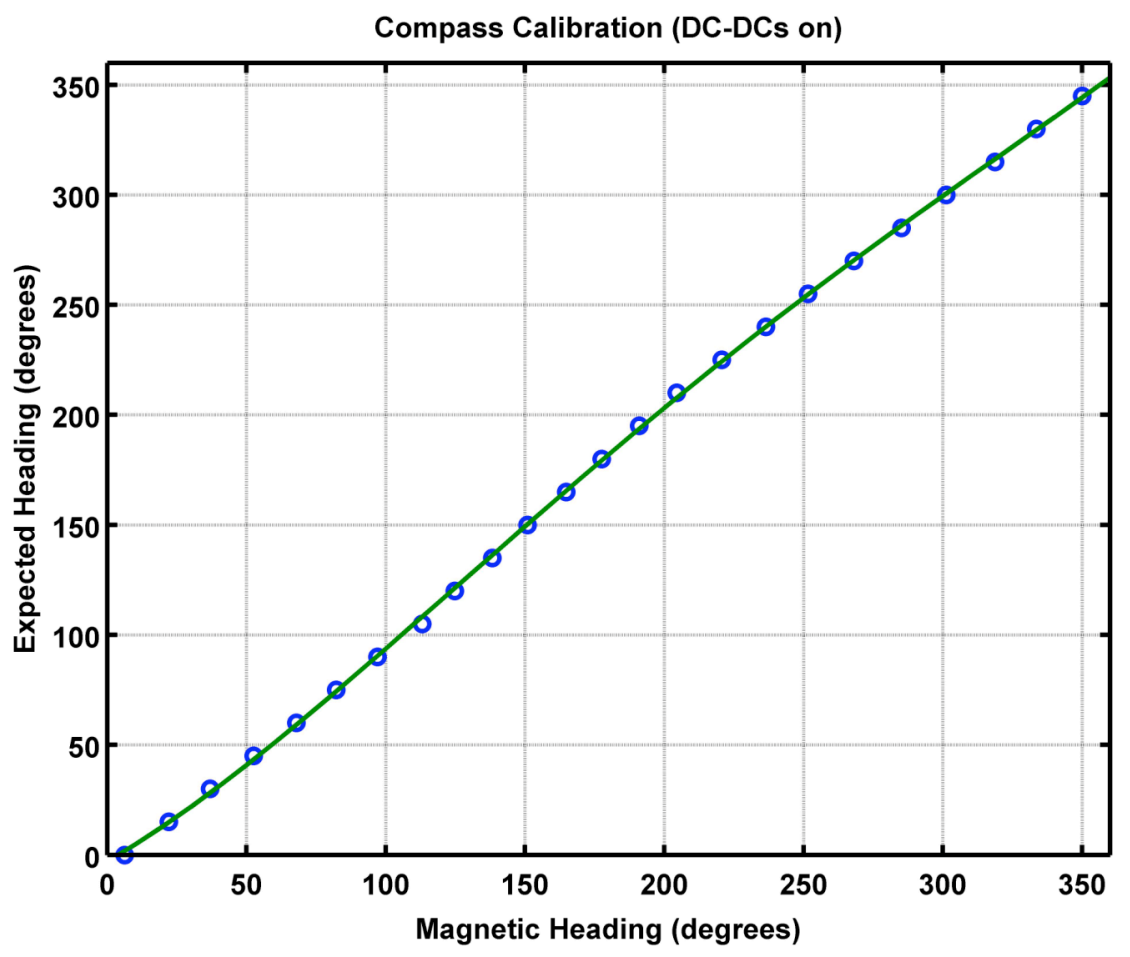

Figure D.10 Compass Calibration (DC-DCs on) data plotted with a third degree polynomial fit. 


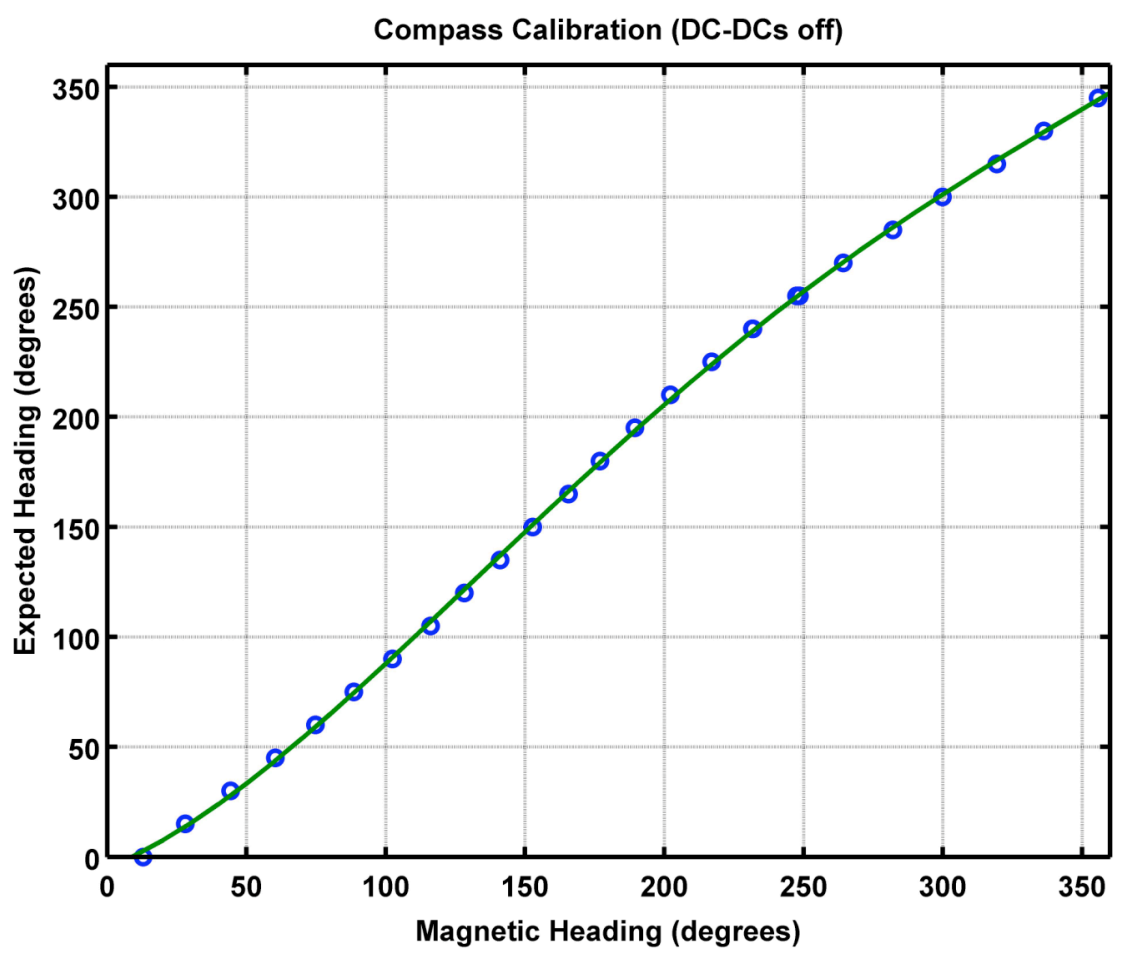

Figure D.11 Compass Calibration (DC-DCs off) data plotted with a third degree polynomial fit.

A summary of the calibrations of the accelerometers, rate gyros, and compass tilt axes is given in Table D.5.

\begin{tabular}{|l|ll|}
\hline \multicolumn{3}{|c|}{ DAS Tilt Sensor Calibration Summary } \\
\hline \multicolumn{1}{|c|}{ Instrument Axis } & \multicolumn{2}{c|}{ Calibration } \\
\hline Accelerometer A & $a=2.959 \times 10^{-5} C+0.9964 \quad[\mathrm{~g}]$ \\
\hline Accelerometer B & $a=2.896 \times 10^{-5} C+0.0108$ & {$[\mathrm{~g}]$} \\
\hline Accelerometer C & $a=2.838 \times 10^{-5} C+0.0097$ & {$[\mathrm{~g}]$} \\
\hline Rate Gyro A & $\Omega=2.556 \times 10^{-5} C$ & {$[\mathrm{rad} / \mathrm{s}]$} \\
\hline Rate Gyro B & $\Omega=1.558 \times 10^{-5} C$ & {$[\mathrm{rad} / \mathrm{s}]$} \\
\hline Rate Gyro C & $\Omega=1.553 \times 10^{-5} C$ & {$[\mathrm{rad} / \mathrm{s}]$} \\
\hline Compass Tilt B & $\theta=1.089 \theta_{r}+0.1474$ & {$[\mathrm{deg}]$} \\
\hline Compass Tilt C & $\theta=1.071 \theta_{r}-0.6521$ & {$[\mathrm{deg}]$} \\
\hline
\end{tabular}

Table D.5 Summary of tilt sensor orientations and calibrations. 
Where $a$ is acceleration in the units of gravitational acceleration, $g, C$ is counts, $\theta$ is calibrated angle in degrees, $\Omega$ is angular rate of change in radians per second, and $\theta_{r}$ is the reported compass tilt angle in degrees. 


\section{Appendix E}

\section{ASIS Drift Model}

The low frequency drift response of ASIS due to the combined forcing of winds and currents was computed as follows. Effective drag coefficients in air and water for our specific ASIS configuration were computed using

$$
\alpha=\frac{1}{2} \rho \sum_{n} C_{d_{n}} A_{n}
$$

where $\rho$ is the density of the medium (air or water), $C_{d_{n}}$ is the drag coefficient, and $A_{n}$ is the cross-sectional area of $n^{\text {th }}$ structural element of ASIS in air or water. The $\alpha$ 's were found to be 0.452 and $657 \frac{\mathrm{N}}{(\mathrm{m} / \mathrm{s})^{2}}$ for air and water, respectively. Then the air and waterside drag on the buoy is given by

$$
\mathbf{D}=\alpha *\left|U_{r}\right| * \mathbf{U}_{r}
$$

where $\mathbf{D}$ is the drag, and $\mathbf{U}_{r}$ is the velocity of the air or water relative to the buoy. We also incorporated water drag on the tether using

$$
\mathbf{D}_{T}=\alpha U_{n}^{2} \mathbf{u}_{n}
$$

where $\mathbf{D}_{T}$ is the drag on the tether, $\alpha$ is computed as above for the tether, $U_{n}$ is the relative water velocity normal to the tether, and $\mathbf{u}_{n}$ is the unit vector normal to the tether. Assuming that the tether extension is a linear function of the tension, the tension in the tether is given by

$$
\begin{array}{ll}
\mathbf{T}=-k^{*} R^{\prime *} \mathbf{u}_{t} & \text { for } R^{\prime}>0 \\
\mathbf{T}=0 & \text { for } R^{\prime} \leq 0
\end{array}
$$


where $\mathbf{T}$ is the tension, $k$ is the spring constant, $R^{\prime}$ is the tether extension beyond its unstretched length, and $\mathbf{u}_{t}$ is the unit vector tangent to the tether. The equation of motion for the buoy then is

$$
M_{v} \frac{d \mathbf{V}}{d t}=\mathbf{D}_{w}+\mathbf{D}_{a}+\mathbf{D}_{T}+\mathbf{T}
$$

where $\mathbf{D}_{w}$ and $\mathbf{D}_{a}$ are the water and air-side drags, respectively, $\mathbf{V}$ is the horizontal velocity of the buoy relative to the earth, and $M_{v}$ is the sum of the buoy's inertial and added mass. It is straightforward to integrate equation (E.5) for the buoy velocity, starting from an initial value. 


\section{Appendix F}

\section{Tilting Table}

A mechanically-tilting table was designed and constructed in order to test the sodar's sensitivity to rotational motion. It also provided a convenient way to calibrate the rate gyros inside the DAS.

The tilting table was designed by Ben Allen (Woods Hole Oceanographic Institution), and assembled by the author. The table is made up of two similar pieces, a base and a tabletop, that are held together by a shaft that rotates inside a pair of bearings. The bearings allow the tabletop to move freely. The tabletop's movement is driven by a motor that is mounted on the table's base. An aluminum arm connects the moving shaft of the motor to the top of the table. The arm can be adjusted to allow for a variable range of tilt (the maximum is about 20 degrees). All table parts are made of aluminum, with the exception of the top platform which is made of marine wood. A computerized drawing of the tilting table is shown below in Figure F.1. 


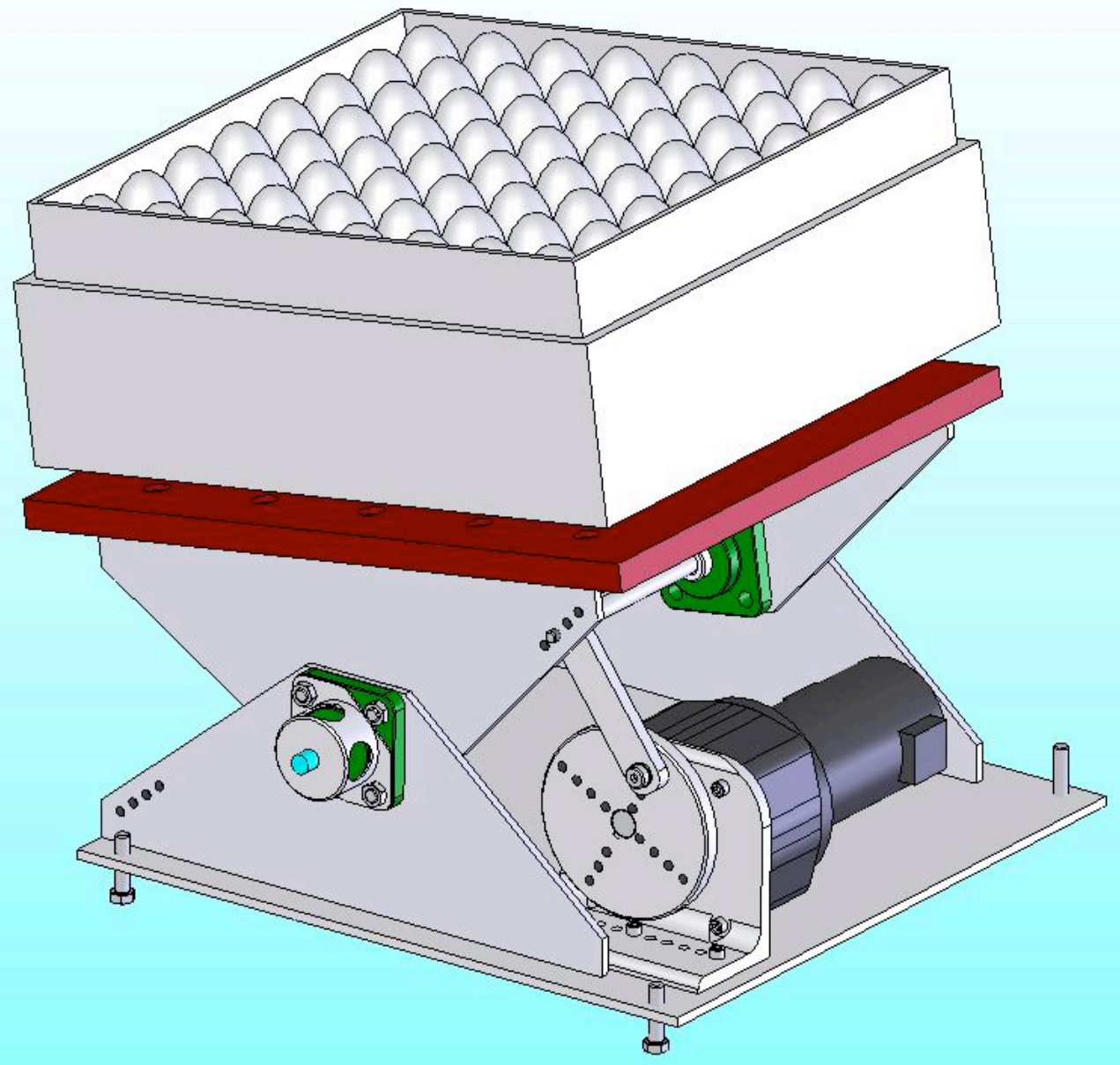

Figure F.7 A computerized drawing of the tilting table (figure courtesy of B. Allen).

The motor is a Leeson DC straight shaft motor (model No: CM34D25NZ61C) that is driven by a Leeson DC control gearbox. The motor can rotate as fast as 14 RPM, which allows for rotational periods in the range of 3 to 20 seconds. This is ideal for our application because this frequency range encompasses the band of wave-induced buoy motion.

In order to measure the tilt and period of the table, a Honeywell RV6 potentiometer is mounted on the table's shaft. A special mounting was designed at WHOI to hold the body of the potentiometer fixed to the base of the table while allowing the shaft to move. 
The shaft of the potentiometer moves with the shaft of the tilting table. The potentiometer's output is logged by a 10-bit Dataq A-D converter (DI-194 RS).

The potentiometer was calibrated by statically tilting the table and reading its output using the Dataq A/D converter. The potentiometer's voltage output divided by its reference voltage was then compared to the tilt of the corresponding calibrated accelerometer as well as the tilt measured by a digital protractor. Both calibrations were within a few percent of one another, so we take the average to be the potentiometer calibration. The result is

$$
\theta=\left(\frac{V_{\text {out }}}{V_{\text {ref }}}-0.595\right) / 3.70 \times 10^{-3}
$$

where $\theta$ is angle of tilt (degrees), $V_{\text {out }}$ is output voltage in volts, and $V_{\text {ref }}$ is a reference voltage in volts. Plots of the results of the calibration using the digital protractor and the accelerometers are shown in Figures F.2 and F.3, respectively. 


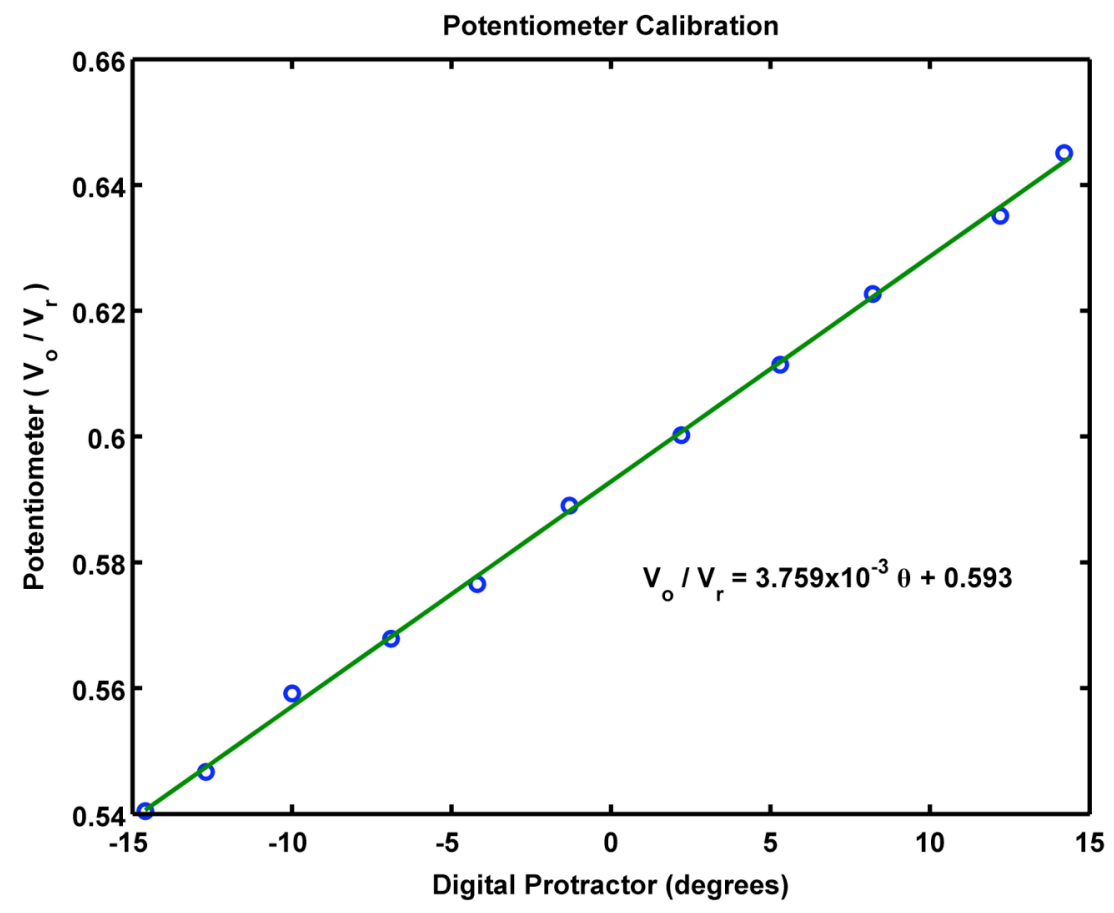

Figure F.8 Potentiometer Calibration with the digital protractor plotted with a linear fit.

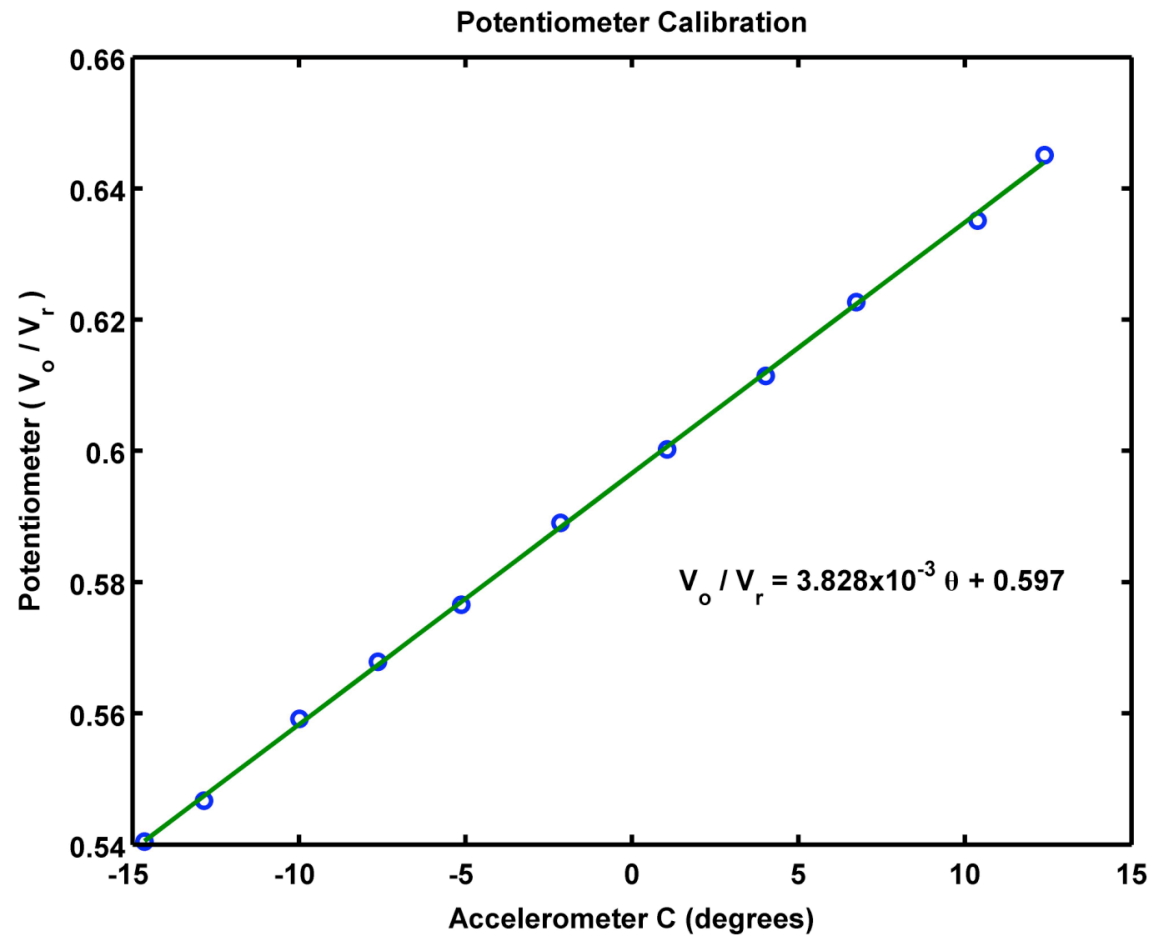

Figure F.9 Potentiometer Calibration with Accelerometer C plotted with a linear fit. 
A plot of the spectrum of the potentiometer for a table period of approximately 8 seconds is shown in Figure F.4. A second harmonic is apparent in the neighborhood of $0.3 \mathrm{~Hz}$. This is evidence that the table's motion is not purely sinusoidal. However, the ratio of the area under the peak of the second harmonic to the area under the peak of the first harmonic is $1.27 \times 10^{-2}$. A similar second harmonic can also be seen in the output of the rate gyros (see Appendix D).

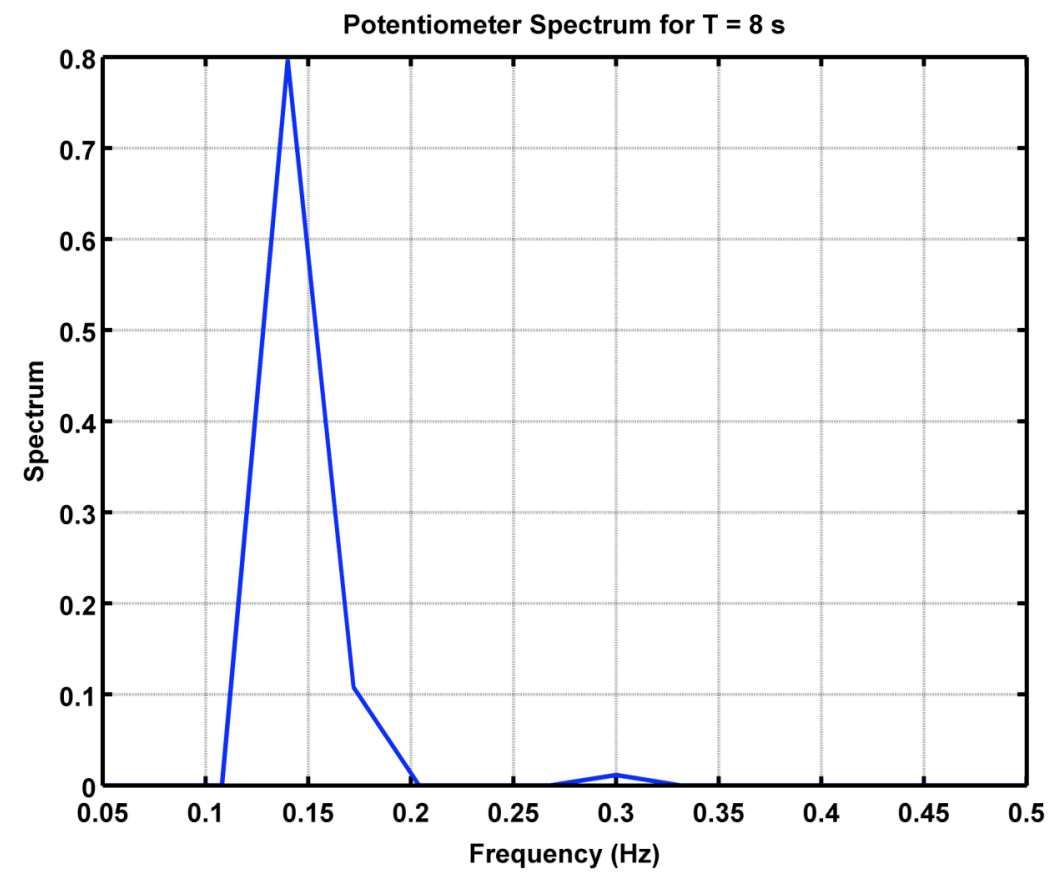

Figure F.10 A plot of the potentiometer spectrum for a table period of approximately 8 seconds. 


\section{Appendix G}

\section{Probability density function of incremental motion}

As discussed in section 5.4, we are interested in the effect on the received signal-to-noise ratio of three motions: heave, surge and pitch. In this appendix, we compute the probability density function (pdf) for increments in heave, $d h$, over a time interval $\Delta \mathrm{t}$ $(=2 R / C)$, corresponding to the round-trip travel time for a given range cell, $R$. The probability density for all three motions has the same form, and hence it is sufficient to determine it for heave alone.

If we know the pdf of $d h=h(t+\Delta t)-h(t)$ for a given time lag, we can predict how the sodar will perform in different sea states. Unfortunately, we did not make wave height measurements from ASIS, and so cannot directly compute a time series of $d h$. Instead we use wave height spectra $S_{h}(f)$ computed from 20 minute "bursts" taken at the MVCO 12 meter node continuously during 2005. These spectra are used to synthesize time series of $d h$ for each "burst", from which we estimate a pdf.

The 2005 data set consists of 22,689 wave height spectra. In order to keep track of different sea states, we group these into $20 \times 21$ bins of average frequency, $F p$, and significant height, $H_{s}$. The grid is defined by $0.088 \leq F p \leq 0.325(\mathrm{~Hz})$ and $0.343 \leq H_{S} \leq 4.46(\mathrm{~m})$ with step sizes of $0.0124 \mathrm{~Hz}$ and $0.206 \mathrm{~m}$. $H_{s}$ was taken to be as four times the standard deviation computed by integrating the spectral density of the wave height, while $F p$ was computed as the first moment of the height spectrum. Figure G. 1 shows a scatterplot of the 22,689 values of mean frequency and significant height that occurred. We call this a "Nantucket" distribution, because its shape resembles that of the island. Figure G.2 shows a three-dimensional histogram of the number of spectra in each $\left(\bar{f}, H_{s}\right)$ bin. We find that 168 bins are occupied. 


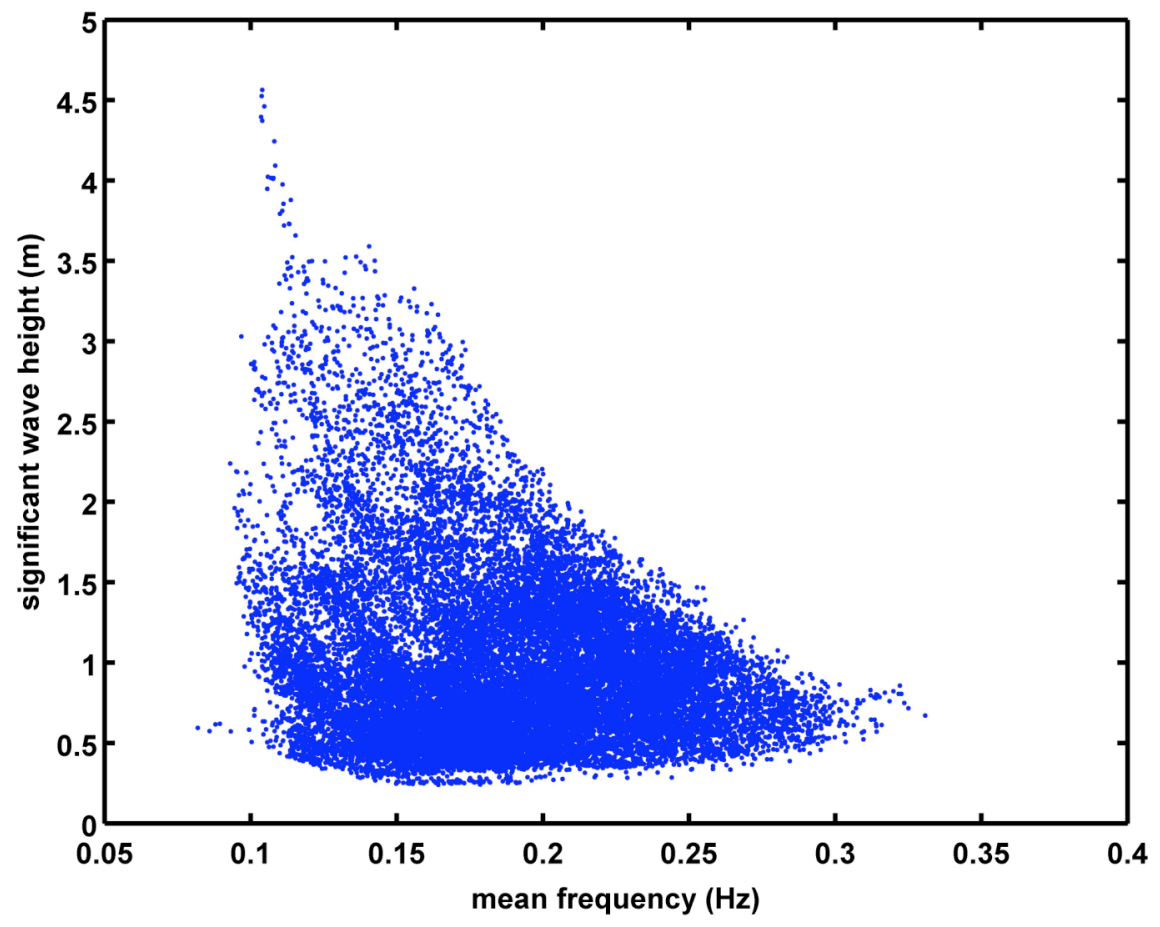

Figure G.11 The "Nantucket" distribution of 28,000 sea surface height spectra.

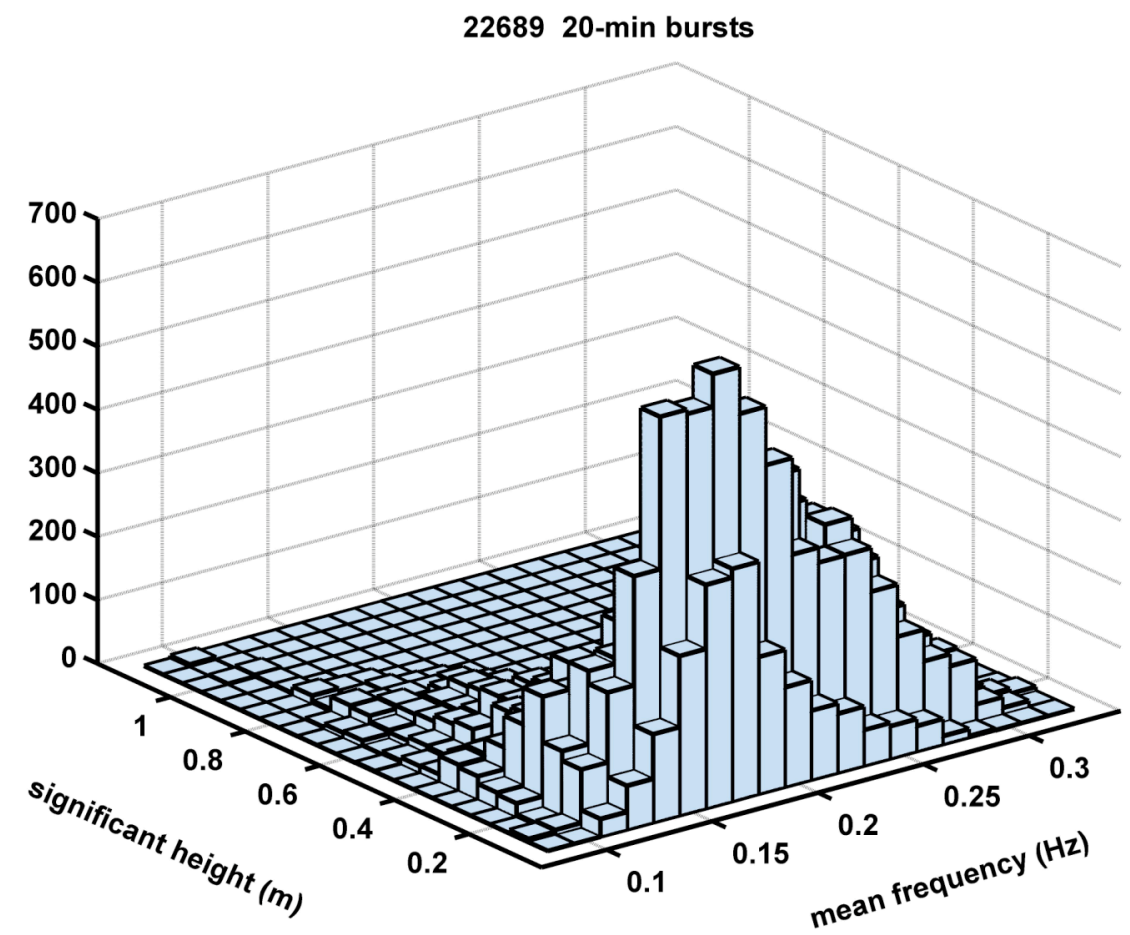

Figure G.12 Pdf of sea surface height spectra. 
For each of the 168 sea states, we synthesize a 20 minute random time series (sampled at $1 \mathrm{~Hz}$ ) of sea surface height, $h(t)$. Defining 20 uniformly-spaced time lags, $\Delta \mathrm{t}$, from $50 \mathrm{~ms}$ to $1 \mathrm{~s}$ (corresponding to ranges of $8-170 \mathrm{~m}$ ), we compute time series of heave increments $d h=h(t+\Delta t)-h(t)$ for each sea state. Zero-mean Gaussian pdf of the form

$$
P(d h)=\frac{1}{\sigma \sqrt{2 \pi}} e^{-(d h)^{2} / 2 \sigma^{2}}
$$

where $\sigma=\sigma\left(F p, H_{s}, \Delta t\right)$ specifies the width, was fit to each time series, $d h$. As an example, we show results for the three cases of high, mid-range and low sea states defined in Table G.1 for the largest time lag of $\Delta \mathrm{t}=1 \mathrm{~s}$. The corresponding average spectra are shown in Figure G.3.

\begin{tabular}{|c|c|c|c|}
\hline Sea state & $\begin{array}{c}\text { Mean frequency } \\
(\mathbf{H z})\end{array}$ & $\begin{array}{c}\text { Significant height } \\
(\mathbf{m})\end{array}$ & Time lag (s) \\
\hline High & 0.10 & 4.46 & 1.00 \\
\hline Mid & 0.20 & 2.20 & 1.00 \\
\hline Low & 0.27 & 1.17 & 1.00 \\
\hline
\end{tabular}

Table G.1 Summary of three sea states.

Figure G.4 shows how close the empirical pdf is to Gaussian, and Figure G.5 shows the standard deviation as a function of time lag. These figures demonstrate that the distributions are very close to Gaussian with varying widths (standard deviations) that are close to linear in the time lag $\Delta$ t. Although we have shown only 3 cases, the linear dependence on $\Delta \mathrm{t}$ is characteristic of all sea states. The three cases shown were chosen because we expected deviations from linearity at large values of $F p$ and/or $H_{s}$. Small departures from linearity were observed for large values of $\Delta \mathrm{t}$, but overall the behavior of $\sigma$ is very close to linear in $\Delta t$. 


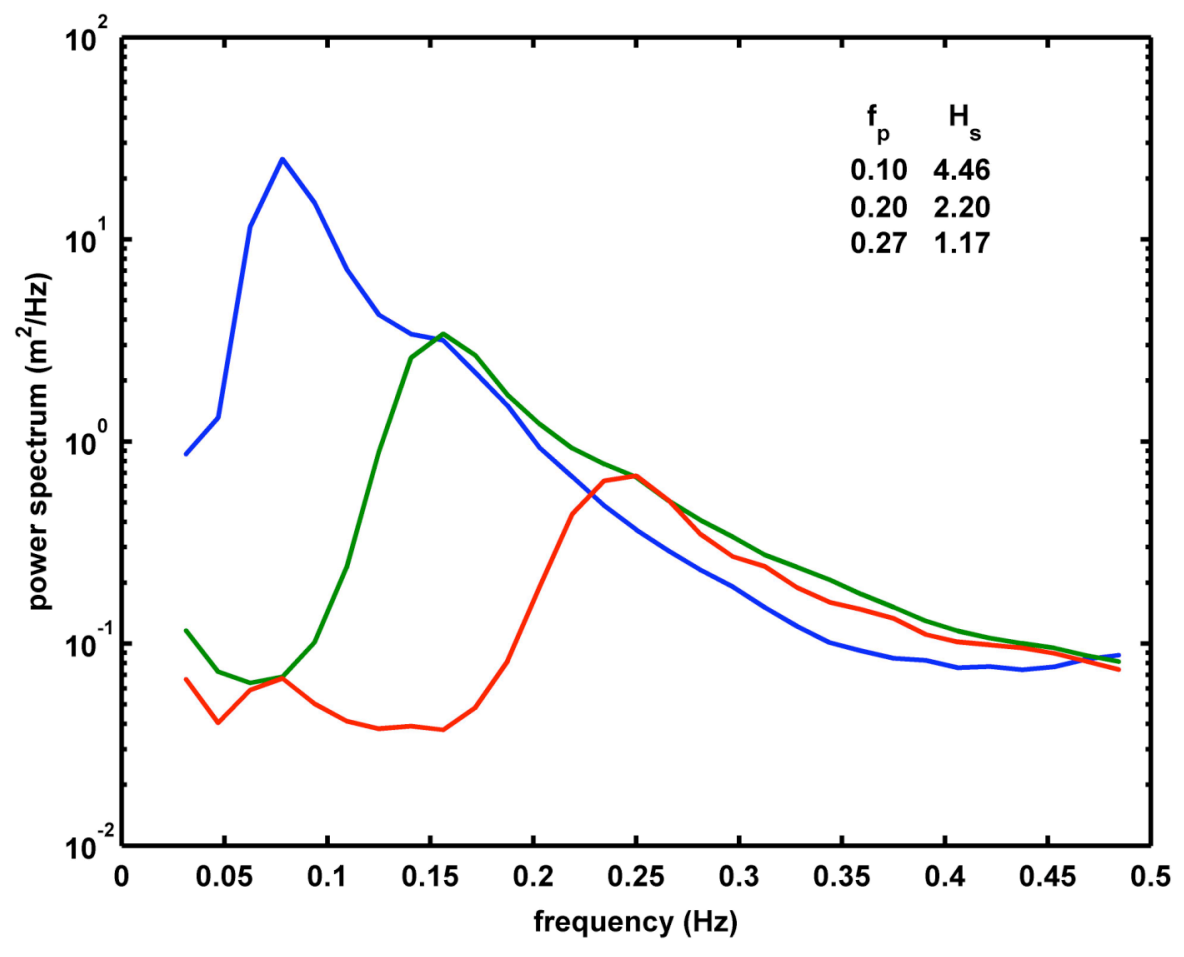

Figure G.13 Spectra of the three sea states defined in Table G.1.

(symbol, $\left.f_{p}, H_{s}, \Delta t\right):$ ('x',0.10,4.4,1), ('o',0.20,2.2,1), ('+',0.27,1.2,1)

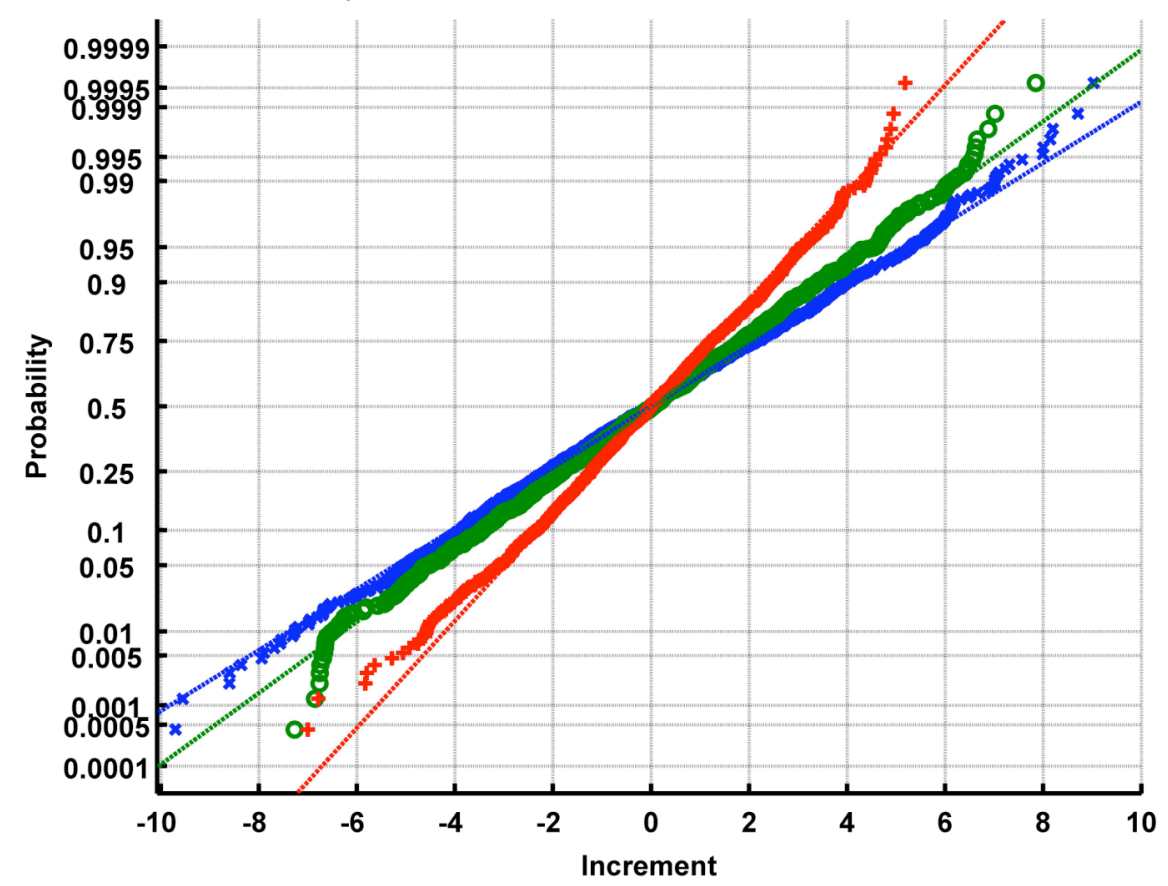

Figure G.14 Comparison of observations to a normal (Gaussian) distribution for the three sea states and time lag defined in Table G.1. 


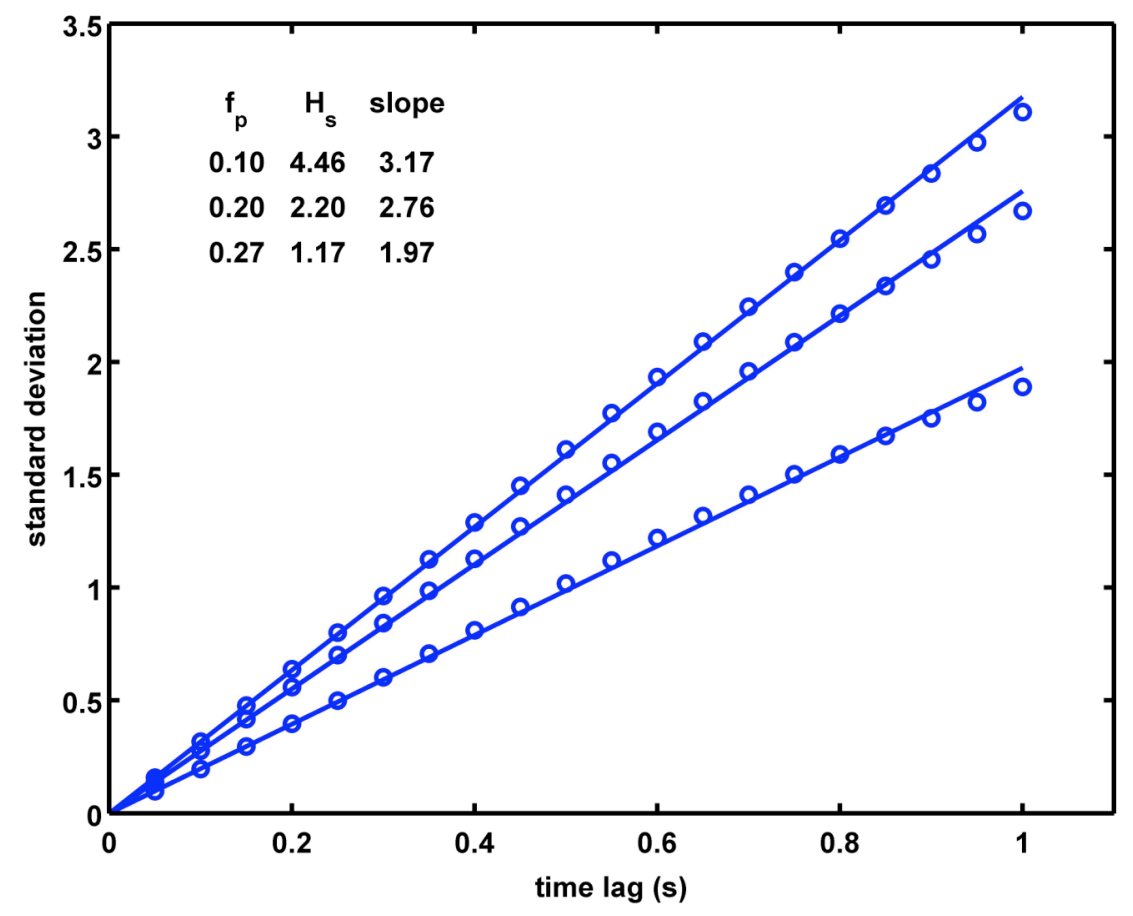

Figure G.15 Standard deviation as a function of time lag for three defined sea states.

Hence the standard deviation, $\sigma$, of the pdf of $d h$ can be written as

$$
\sigma\left(F p, H_{s} \Delta t\right)=\beta\left(F p, H_{s}\right) \Delta t
$$

$\beta$ was then computed for each sea state $\left(F p, H_{s}\right)$. 